\title{
HAMILTON-JACOBI EQUATIONS FOR CONTROL PROBLEMS OF PARABOLIC EQUATIONS
}

\author{
Sophie GombaO ${ }^{1}$ And JeAn-Pierre RAYMOnd ${ }^{1}$
}

\begin{abstract}
We study Hamilton-Jacobi equations related to the boundary (or internal) control of semilinear parabolic equations, including the case of a control acting in a nonlinear boundary condition, or the case of a nonlinearity of Burgers' type in $2 \mathrm{D}$. To deal with a control acting in a boundary condition a fractional power $(-A)^{\beta}$ - where $(A, D(A))$ is an unbounded operator in a Hilbert space $X$ - is contained in the Hamiltonian functional appearing in the Hamilton-Jacobi equation. This situation has already been studied in the literature. But, due to the nonlinear term in the state equation, the same fractional power $(-A)^{\beta}$ appears in another nonlinear term whose behavior is different from the one of the Hamiltonian functional. We also consider cost functionals which are not bounded in bounded subsets in $X$, but only in bounded subsets in a space $Y \hookrightarrow X$. To treat these new difficulties, we show that the value function of control problems we consider is equal in bounded sets in $Y$ to the unique viscosity solution of some Hamilton-Jacobi-Bellman equation. We look for viscosity solutions in classes of functions which are Hölder continuous with respect to the time variable.
\end{abstract}

Mathematics Subject Classification. 49K20, 49L25.

Received November 16, 2004.

\section{INTRODUCTION}

In this paper we study the uniqueness and existence of viscosity solution of the Hamilton-Jacobi-Bellman equation

$$
\begin{gathered}
-\frac{\partial v}{\partial t}(t, x)-\left(D_{x} v(t, x) \mid A x\right)_{X}+\left(D_{x} v(t, x) \mid(-A)^{\beta} F(t, \Lambda x)\right)_{X} \\
+H\left(t, x,(-A)^{\beta} D_{x} v(t, x)\right)=0 \quad \text { in }(0, T) \times X, \\
v(T, x)=g(x) \quad \text { in } X .
\end{gathered}
$$

In this setting $X$ is a real Hilbert equipped with the inner product $(\cdot \mid \cdot)_{X}$ and the norm $|\cdot|_{X}, A$ is an unbounded operator with domain $D(A)$ in $X$, it is supposed to be self-adjoint and strictly dissipative in $X,(-A)^{\beta}$ is the $\beta$-fractional power of $(-A)$, and $0 \leq \beta \leq \frac{1}{2}, \Lambda$ is a bounded linear operator from $D\left((-A)^{\alpha}\right)$ into $X_{0}$ with $0 \leq \alpha<\frac{1}{2}, X_{0}$ is another real Hilbert space equipped with the inner product $(\cdot \mid \cdot)_{X_{0}}$ and the norm $|\cdot|_{X_{0}}$,

Keywords and phrases. Hamilton-Jacobi-Bellman equation, boundary control, semilinear parabolic equations.

1 Laboratoire MIP, UMR CNRS 5640, Université Paul Sabatier, 31062 Toulouse Cedex 4, France; gombao@cict.fr; raymond@mip.ups-tlse.fr 
$F \in C\left([0, T] \times X_{0} ; X\right)$, the Hamiltonian functional $H$ is continuous in $[0, T] \times X \times X$, and the mapping $g$ is Lipschitz continuous in $X$. Precise assumptions are stated in Section 2.

Equation (1.1) is related to optimal control problems of semilinear parabolic equations (including in particular the case where the control acts in a nonlinear boundary condition). More precisely, for all $t \in[0, T]$ and $x \in X$, consider an optimal control problem of the form

$$
\min \{J(t, u, y) \mid u \in \mathcal{M}(t, T ; U) \text { and }(y, u) \text { is solution of equation }(1.2)\},
$$

where the cost functional $J$ is defined by

$$
J(t, y, u)=\int_{t}^{T} L(r, y(r), u(r)) \mathrm{d} r+g(y(T)),
$$

and the state equation is

$$
y^{\prime}=A y+(-A)^{\beta}[B u-F(\cdot, \Lambda y)], \quad y(t)=x .
$$

The control space $\mathcal{M}(t, T ; U)$ is a set of bounded measurable functions with values in $U$, and $U$ is a bounded subset in $X_{\Gamma}, X_{\Gamma}$ is a Banach space, $B \in \mathcal{L}\left(X_{\Gamma}, X\right)$. In Section 3 we prove that equation (1.1) admits at most one viscosity solution. In Section 4 , we prove that the value function of problem $\left(\mathcal{P}_{t, x}\right)$ is the unique viscosity solution of equation (1.1), when $H$ is defined by

$$
H(t, x, p)=\sup _{u \in U}\left[-(p \mid B u)_{X}-L(t, x, u)\right] .
$$

Applications are discussed in Section 5. Before presenting what is new in the present paper, observe that by setting

$$
\mathbf{H}\left(t, x,(-A)^{\beta} D_{x} v(t, x)\right)=H\left(t, x,(-A)^{\beta} D_{x} v(t, x)\right)+\left((-A)^{\beta} D_{x} v(t, x) \mid F(t, \Lambda x)\right)_{X},
$$

equation (1.1) can be written in the form

$$
-\frac{\partial v}{\partial t}(t, x)-\left(D_{x} v(t, x) \mid A x\right)_{X}+\mathbf{H}\left(t, x,(-A)^{\beta} D_{x} v(t, x)\right)=0 \quad \text { in }(0, T) \times X .
$$

Equation (1.3) seems to be simpler to handle than equation (1.1). However assumptions on $F(t, \Lambda x)$ and on $H(t, x, p)$ are different and we cannot simplify the presentation of the paper by considering equation (1.3) (see e.g. the estimates involving $H$ and $F$ in the proof of Th. 3.5).

During the eighties and the nineties several fundamental advances have been made in the study of HamiltonJacobi equation in infinite dimension. These equations were first studied by Barbu and Da Prato (see e.g. [2]), mainly in classes of convex functions. The method of viscosity solutions has been extended to infinite dimension by Crandall and Lions in a series of papers [10-14]. All these papers correspond to the case when $\beta=0$. Other contributions are due to Cannarsa and Frankowska [5], Ishii [20], Soner [27], Tataru [28,29], Crandall and Lions $[15,16]$, Cannarsa and Tessitore [6-9] in order to deal with boundary controls. In particular equations of the form (1.3) with $0<\beta<\frac{1}{2}$ are studied in [6], [8] to treat Neumann boundary controls. The case of Dirichlet controls is considered in [7,9], it corresponds to the situation when $\frac{1}{2}<\beta<1$ and has to be studied independently. More recently the case of the Navier-Stokes equations has been studied in $[18,26]$.

The main motivation of the present paper is to characterize the value function of control problems governed by semilinear parabolic equations, including the case of equations with a nonlinear boundary condition, or the case of nonlinearity of Burgers' type in two dimension, and with cost functionals whose growth is quadratic or even higher than quadratic. For example we study the case of partial differential equations with nonlinear boundary conditions of the form:

$$
\left.\frac{\partial y}{\partial t}-\Delta y+y=f \quad \text { in }\right] t, T\left[\times \Omega, \quad \frac{\partial y}{\partial n}+\widehat{h}(y)=u \quad \text { on }\right] t, T[\times \Gamma, \quad y(t)=x \quad \text { in } \Omega,
$$


with cost functionals of the type

$$
\widehat{J}(t, y, u)=\int_{t}^{T} \widehat{L}(r, y(r), u(r)) \mathrm{d} r+\widehat{g}(y(T)),
$$

where $\widehat{h}$ is any regular nondecreasing function obeying $\widehat{h}(0)=0$, and where $\widehat{L}$ and $\widehat{g}$ may be quadratic cost functionals. Many thermal processes lead to the kind of model corresponding to equation (1.4) (see [23]). The papers mentioned above do not include this model in their possible applications. If the initial condition $x$ belong to $X=L^{2}(\Omega)$, equation (1.4) is well posed and it admits a unique weak solution belonging to $C([0, T] ; X)$ (the solution also belongs to $\left.L^{2}\left(0, T ; H^{1}(\Omega)\right)\right)$. We can write equation (1.4) in the form

$$
y^{\prime}=A y+(-A)^{\beta}[B u-\widehat{F}(\cdot, \Lambda y)], \quad y(t)=x,
$$

by defining $\Lambda$ as the trace mapping on $\Gamma$ :

$$
\Lambda:\left.y \longmapsto y\right|_{\Gamma} .
$$

In this example $\Lambda$ is bounded from $H^{2 \alpha}(\Omega)=D\left((-A)^{\alpha}\right)$ into $X_{0}=L^{2}(\Gamma)$ for all $\frac{1}{4}<\alpha<\frac{1}{2}, D(A)=\{y \in$ $\left.H^{2}(\Omega) \mid \frac{\partial y}{\partial n}=0\right\}, A y=\Delta y$, and we have to take $\frac{1}{4}<\beta<\frac{1}{2}$. For a parabolic equation with a nonlinearity of Burgers' type we can take $\beta=\frac{1}{2}$. Let us denote by $y_{t, x, u}$ the solution to equation (1.5). To characterize the value function $\widehat{v}(t, x)$ of the problem

$$
\min \{\widehat{J}(t, u, y) \mid u \in \mathcal{M}(t, T ; U) \text { and }(y, u) \text { is solution of equation }(1.5)\},
$$

we have to study the dependence of $y_{t, x, u}$ and of $\Lambda y_{t, x, u}$ with respect to $t$ and to $x$. Due to the nonlinear term in equation (1.5), we can prove continuity properties for $y_{\cdot, x, u}$ and $\Lambda y_{\cdot, x, u}$ and Lipschitz properties for $y_{t, \cdot, u}$ and $\Lambda y_{t,, u}$ when the initial condition $x$ stays in bounded subsets in $Y$, for a space $Y \hookrightarrow X$, but these properties are not true if we consider only bounded subsets in $X$. Therefore it is natural to study the properties of the value function $\widehat{v}(t, x)$ when $x$ remains in bounded subsets of $Y$, and to look for solutions to equation (1.4) in a space of the type $C([0, T] ; Y)$ or at least $L_{w}^{\infty}(0, T ; Y)$ (the space of bounded and weakly measurable functions from $(0, T)$ into $Y)$.

Another difficulty comes from the cost functional. In the literature on Hamilton-Jacobi-Bellman equations, it is often assumed that the cost functionals either are bounded or satisfy a linear growth condition $[6,8,18,20]$. Thus the case of quadratic cost functionals is not treated in these papers.

To overcome the two difficulties mentioned above, the one coming from the nonlinearity in the state equation and the other one due to the growth condition of the cost functional, we suggest to proceed as follows. First, we show that, for an initial condition in $B_{Y}\left(M_{0}\right)$ (the ball in $Y$ centered at the origin and with radius $M_{0}$ ), the solution $y$ of equation (1.2) satisfies $y(\cdot) \in B_{Y}\left(R_{T}\right)$ in $(t, T)$ for some $R_{T}=R\left(M_{0}, T\right)$ which can be explicitly estimated independently of $t \in(0, T)$. Next, we associate with the mappings $\widehat{L}(t, \cdot, u), \widehat{g}$ and $\widehat{F}(t, \Lambda \cdot)$, other mappings $L(t, \cdot, u), g$ and $F(t, \Lambda \cdot)$ which are identical to the previous ones in the ball $B_{Y}\left(R_{T}\right)$, but which satisfies some global boundedness and Lipschitz properties. Let us consider the problem $\left(\mathcal{P}_{t, x}\right)$ - the one introduced at the beginning of the introduction - defined with $L(t, \cdot, u), g$ and $F(t, \Lambda \cdot)$. We are able to show that value function $v(t, x)$ of problem $\left(\mathcal{P}_{t, x}\right)$ obeys $\widehat{v}(t, x)=v(t, x)$ for $t \in(0, T)$ and $x \in B_{Y}\left(R_{T}\right)$. We show that $v$ is the unique viscosity solution of the Hamilton-Jacobi equation (1.1). Thus $\widehat{v}$ is not the viscosity solution to equation (1.1), but it is equal to the viscosity solution of equation (1.1) in bounded sets in $(0, T) \times Y$.

Sections 2, 3 and 4 are devoted to the study of equation (1.2), equation (1.1), and the value function of problem $\left(\mathcal{P}_{t, x}\right)$. In these sections, only the mappings $L(t, \cdot, u), g$ and $F(t, \Lambda \cdot)$ intervene. The assumptions are precisely stated in Section 2. The definition of the mappings $L(t, \cdot, u), g$ and $F(t, \Lambda \cdot)$ from $\widehat{L}(t, \cdot, u), \widehat{g}$ and $\widehat{F}(t, \Lambda \cdot)$ is treated in examples of Section 5 by using projection operators. Three examples are considered. The first one is a control problem for the state equation (1.4), and the two others correspond to problems for a two dimensional scalar equation of Burgers' type. The interest of the third example is to show that the method 
using a projection operator in the cost functional and the state equation is flexible enough to involve different kind of projections adapted to the nonlinearity and to the functionals we have to deal with.

Let us finally mention that the definition of viscosity solutions that we take is not totally standard. Indeed we consider viscosity solutions which are Hölder continuous with respect to the time variable. This Hölder continuity condition, which is a new argument in the definition of viscosity solutions - see Definition 3.2 - plays a major role in the proof of uniqueness to estimate the nonlinear term $F$. A preliminary version of the present paper corresponds to a part of the Ph.D. thesis by the first author [17].

\section{Preliminaries on the evolution equation}

In this section we want to study properties of solutions of the evolution equation

$$
y^{\prime}=A y+(-A)^{\beta}[B u-F(\cdot, \Lambda y)] \quad \text { in }(t, T), \quad y(t)=x,
$$

where $t \in[0, T)$.

\subsection{Assumptions}

Throughout the paper we make the following assumptions.

(i) The unbounded operator $A$, with domain $D(A)$ in $X$, is a closed and densely defined selfadjoint operator in $X$, such that $(A x \mid x)_{X} \leq-\omega|x|_{X}^{2}$ for all $x \in D(A)$, where $\omega>0$.

(ii) $B \in \mathcal{L}\left(X_{\Gamma}, X\right)$.

(iii) The linear operator $\Lambda$ is bounded from $D\left((-A)^{\alpha}\right)$ into $X_{0}$ for some $\alpha \in\left[0, \frac{1}{2}\right.$, that is:

$$
|\Lambda x|_{X_{0}} \leq C_{\alpha}\left|(-A)^{\alpha} x\right|_{X} \quad \text { for all } x \in D\left((-A)^{\alpha}\right) .
$$

The exponent $\beta \in\left[0, \frac{1}{2}\right]$ is given fixed.

(iv) $F$ is a continuous mapping from $[0, T] \times X_{0}$ into $X$, which satisfies:

$$
|F(t, x)-F(t, y)|_{X} \leq K_{F}|x-y|_{X_{0}} \text {, and }|F(t, x)|_{X} \leq M_{F},
$$

for all $t \in[0, T]$, and all $x, y \in X_{0}$. Moreover, there exists $\left.\left.\eta_{1} \in\right] 0,1\right]$ such that:

$$
|F(t, x)-F(s, x)|_{X} \leq M_{1, F}\left(1+|x|_{X_{0}}\right)|t-s|^{\eta_{1}} .
$$

In addition, we assume that either $\beta<\frac{1}{2}$, or $\beta=\frac{1}{2}$ and

$$
\begin{aligned}
& D\left((-A)^{\frac{1}{2}}\right) \hookrightarrow X_{0}, \\
& |F(t, x)|_{D\left((-A)^{\beta_{0}}\right)} \leq M\left(\beta_{0},|x|_{D\left((-A)^{\frac{1}{2}}\right)}\right) \quad \text { for all } t \in[0, T] \text { and all } x \in D\left((-A)^{\frac{1}{2}}\right), \\
& (-A)^{\beta_{0}} B \in \mathcal{L}\left(X_{\Gamma}, X\right), \quad \text { for some } 0<\beta_{0}<\beta=\frac{1}{2},
\end{aligned}
$$

where $M\left(\beta_{0},|x|_{D\left((-A)^{\frac{1}{2}}\right)}\right)>0$ only depends on $\beta_{0}$ and $|x|_{D\left((-A)^{\frac{1}{2}}\right.}$.

(v) The control $u$ belongs to $\mathcal{M}(t, T ; U)$, the space of measurable functions from $(t, T)$ into $U$, where $U$ is a nonempty, bounded and closed subset of $X_{\Gamma}$, such that

$$
|u|_{X_{\Gamma}} \leq M_{U} \quad \text { for all } u \in U
$$

We now state assumptions needed in Section 3 to study equation (1.1). 
(vi) The mapping $g \in C(X)$ is Lipschitz continuous and bounded in $X$, i.e.:

$$
|g(x)-g(y)| \leq K_{g}|x-y|_{X} \quad \text { and } \quad|g(x)| \leq M_{g}, \quad \text { for all } x, y \in X
$$

(vii) The Hamiltonian functional $H$ satisfies

$$
|H(t, x, p)-H(s, y, q)| \leq K_{H}\left(|t-s|^{\eta_{2}}+|x-y|_{X}+|p-q|_{X}\right)
$$

In Section 4, we make the following additional assumption.

(viii) The Hamiltonian functional $H:[0, T] \times X \times X \rightarrow \mathbb{R}$ is defined by:

$$
H(t, x, p)=\sup _{u \in U}\left[-(p \mid B u)_{X}-L(t, x, u)\right]
$$

where the functional $L \in C([0, T] \times X \times U)$ satisfies:

$$
|L(t, x, u)-L(s, y, u)| \leq K_{L}\left(|t-s|^{\eta_{2}}+|x-y|_{X}\right) \quad \text { and } \quad|L(t, x, u)| \leq M_{L},
$$

for all $t, s \in[0, T]$, all $x, y \in X$, and all $u \in U$, with $0<\eta_{2} \leq 1$.

Observe that if $H$ is defined by (2.8) and if $L$ satisfies the estimate stated in (viii), then

$$
|H(t, x, p)-H(s, y, q)| \leq K_{H}\left(|t-s|^{\eta_{2}}+|x-y|_{X}+|p-q|_{X}\right)
$$

with $K_{H}=\max \left(K_{L},\|B\| M_{U}\right)$. Thus assumption (vii) is automatically satisfied in that case.

Due to assumption (i), $(A, D(A))$ is the infinitesimal generator of a strongly continuous analytic semigroup of contractions on $X$ which satisfies

$$
\left\|\mathrm{e}^{t A}\right\|_{\mathcal{L}(X)} \leq \mathrm{e}^{-\omega t}
$$

Moreover (see [19], Th. 1.4.3, Chap. 1 and [3], Prop. 5.1, Chap. 1), for all $\delta \geq 0$, there exists a constant $M_{\delta}$ such that, for all $t>0$ :

$$
\left\|(-A)^{\delta} \mathrm{e}^{A t}\right\|_{\mathcal{L}(X)} \leq M_{\delta} t^{-\delta}
$$

If $0<\delta \leq 1$, and $x \in D\left((-A)^{\delta}\right)$, we have:

$$
\left|\left(\mathrm{e}^{A t}-I\right) x\right|_{X} \leq \frac{1}{\delta} M_{1-\delta} t^{\delta}\left|(-A)^{\delta} x\right|_{X}
$$

Besides, for all $\delta<\gamma$ and all $x \in D\left((-A)^{\gamma}\right)$, one has:

$$
\left|(-A)^{\delta} x\right|_{X} \leq M_{\delta, \gamma}\left|(-A)^{\gamma} x\right|_{X}^{\frac{\delta}{\gamma}}|x|_{X}^{1-\frac{\delta}{\gamma}}
$$

With Young's inequality the last estimate implies that, for all $\delta \in] 0, \frac{1}{2}[$, and all $\sigma>0$, there exists a constant $C_{\delta, \sigma}$ such that:

$$
\left|(-A)^{\delta} x\right|_{X} \leq \sigma\left|(-A)^{\frac{1}{2}} x\right|_{X}+C_{\delta, \sigma}|x|_{X} \quad \text { for } x \in D\left((-A)^{\frac{1}{2}}\right)
$$




\subsection{Properties and regularities of mild solutions of equation (2.1)}

Theorem 2.1. For all $x \in X$ and all $u \in \mathcal{M}(t, T ; U)$, equation (2.1) admits a unique mild solution $y_{t, x, u}$ in $L^{1}\left(t, T ; D\left((-A)^{\alpha}\right)\right)$, it obeys:

$$
y_{t, x, u}(s)=\mathrm{e}^{(s-t) A} x+(-A)^{\beta} \int_{t}^{s} \mathrm{e}^{(s-r) A}\left[B u(r)-F\left(r, \Lambda y_{t, x, u}(r)\right)\right] \mathrm{d} r,
$$

for all $s \in[t, T]$. Moreover $y_{t, x, u}$ belongs to $C([t, T] ; X)$ and satisfies the estimate

$$
\left\|y_{t, x, u}\right\|_{C([t, T] ; X)} \leq C\left(1+|x|_{X}+\|u\|_{L^{\infty}(t, T ; U)}\right)
$$

Proof. Let $t_{1} \in(t, T]$ be such that $C_{\alpha} K_{F} M_{\alpha+\beta} \frac{\left(t_{1}-t\right)^{1-(\alpha+\beta)}}{1-(\alpha+\beta)} \leq 1 / 2$. Let us set $E=L^{1}\left(t, t_{1} ; D\left((-A)^{\alpha}\right)\right)$, and let us show that the mapping

$$
y \longmapsto(\Psi y)(s)=\mathrm{e}^{(s-t) A} x+(-A)^{\beta} \int_{t}^{s} \mathrm{e}^{(s-r) A}[B u(r)-F(r, \Lambda y(r))] \mathrm{d} r,
$$

is a contraction in $E$. First we have:

$$
\begin{aligned}
\int_{t}^{t_{1}}\left|(-A)^{\alpha}(\Psi y)(s)\right| \mathrm{d} s & \leq \int_{t}^{t_{1}}\left|(-A)^{\alpha} \mathrm{e}^{(s-t) A} x\right| \mathrm{d} s+\int_{t}^{t_{1}} \int_{t}^{s}\left|(-A)^{\alpha+\beta} \mathrm{e}^{(s-r) A}[B u(r)-F(r, \Lambda y(r))]\right| \mathrm{d} r \mathrm{~d} s \\
& \leq M_{\alpha}|x|_{X} \frac{\left(t_{1}-t\right)^{1-\alpha}}{1-\alpha}+M_{\alpha+\beta} \int_{t}^{t_{1}} \int_{t}^{s} \frac{1}{(s-r)^{\alpha+\beta}}\left[\|B\| M_{U}+M_{F}\right] \mathrm{d} r \mathrm{~d} s \\
& \leq M_{\alpha}|x|_{X} \frac{\left(t_{1}-t\right)^{1-\alpha}}{1-\alpha}+M_{\alpha+\beta}\left[\|B\| M_{U}+M_{F}\right] \frac{\left(t_{1}-t\right)^{2-(\alpha+\beta)}}{[1-(\alpha+\beta)][2-(\alpha+\beta)]}
\end{aligned}
$$

Thus, if $y \in E, \Psi y$ belongs to $E$. Moreover if $y_{1}, y_{2} \in E$, we can write

$$
\begin{aligned}
\int_{t}^{t_{1}} \mid(-A)^{\alpha}\left(\Psi y_{1}\right)(s)- & \left.(-A)^{\alpha}\left(\Psi y_{2}\right)(s)\right|_{X} \mathrm{~d} s \\
& \leq \int_{t}^{t_{1}} \int_{t}^{s}\left|(-A)^{\alpha+\beta} \mathrm{e}^{(s-r) A}\left[F\left(r, \Lambda y_{1}(r)\right)-F\left(r, \Lambda y_{2}(r)\right)\right]\right|_{X} \mathrm{~d} r \mathrm{~d} s \\
& \leq \int_{t}^{t_{1}} \int_{t}^{s} \frac{M_{\alpha+\beta}}{(s-r)^{\alpha+\beta}} K_{F}\left|\Lambda y_{1}(r)-\Lambda y_{2}(r)\right|_{X_{0}} \mathrm{~d} r \mathrm{~d} s \\
& =K_{F} \int_{t}^{t_{1}}\left|\Lambda y_{1}(r)-\Lambda y_{2}(r)\right|_{X_{0}} \int_{r}^{t_{1}} \frac{M_{\alpha+\beta}}{(s-r)^{\alpha+\beta}} \mathrm{d} s \mathrm{~d} r \\
& \leq C_{\alpha} K_{F} M_{\alpha+\beta} \int_{t}^{t_{1}}\left|(-A)^{\alpha} y_{1}(r)-(-A)^{\alpha} y_{2}(r)\right|_{X} \frac{\left(t_{1}-r\right)^{1-(\alpha+\beta)}}{1-(\alpha+\beta)} \mathrm{d} r \\
& \leq C_{\alpha} K_{F} M_{\alpha+\beta} \frac{\left(t_{1}-t\right)^{1-(\alpha+\beta)}}{1-(\alpha+\beta)} \int_{t}^{t_{1}}\left|(-A)^{\alpha} y_{1}(r)-(-A)^{\alpha} y_{2}(r)\right|_{X} \mathrm{~d} r .
\end{aligned}
$$

Thus $\Psi$ is a contraction in $E$, and it admits a unique fixed point in $E$, which is the unique solution $y$ in $E$ to equation (2.1). In addition $y$ belongs to $C\left(\left[t, t_{1}\right] ; X\right)$ and formula $(2.14)$ is satisfied for all $s \in\left[t, t_{1}\right]$. We can repeat this process on the interval $\left[t_{1}, 2 t_{1}\right]$, and step by step, we prove that equation $(2.1)$ admits a unique solution in $L^{1}\left(t, T ; D\left((-A)^{\alpha}\right)\right)$, which belongs to $C([t, T] ; X)$ and satisfies formula $(2.14)$. 
Proposition 2.2. Assume that $x \in D\left((-A)^{\alpha}\right)$. Then the solution $y_{t, x, u}$ of (2.1) satisfies:

$$
\left|\Lambda y_{t, x, u}(s)-\Lambda x\right|_{X_{0}} \rightarrow 0 \quad \text { uniformly with respect to } u \in \mathcal{M}(t, T ; U) \text { when } s \searrow t \text {. }
$$

Proof. Let $x$ be in $D\left((-A)^{\alpha}\right)$. With inequality (2.2) we have

$$
\left|\Lambda\left(y_{t, x, u}(s)-x\right)\right|_{X_{0}} \leq C_{\alpha}\left|(-A)^{\alpha}\left(y_{t, x, u}(s)-x\right)\right|_{X} .
$$

Due to $(2.14)$, we can write

$$
\begin{aligned}
\left|(-A)^{\alpha}\left(y_{t, x, u}(s)-x\right)\right|_{X} \leq & \left|(-A)^{\alpha}\left(\mathrm{e}^{(s-t) A} x-x\right)\right|_{X} \\
& +\left|(-A)^{\beta+\alpha} \int_{t}^{s} \mathrm{e}^{(s-r) A}\left[B u(r)-F\left(r, \Lambda y_{t, x, u}(r)\right)\right] \mathrm{d} r\right|_{X}
\end{aligned}
$$

We can estimate the two terms in the right hand side of (2.16) as follows:

$$
\left|(-A)^{\alpha}\left(\mathrm{e}^{(s-t) A} x-x\right)\right|_{X}=\left|\left(\mathrm{e}^{(s-t) A}-I\right)(-A)^{\alpha} x\right|_{X},
$$

and

$$
\left|(-A)^{\beta+\alpha} \int_{t}^{s} \mathrm{e}^{(s-r) A}\left[B u(r)-F\left(r, \Lambda y_{t, x, u}(r)\right)\right] \mathrm{d} r\right|_{X} \leq M_{\alpha+\beta} \frac{(s-t)^{1-(\alpha+\beta)}}{1-(\alpha+\beta)}\left(M_{F}+\|B\| M_{U}\right) .
$$

The two terms (2.17) and (2.18) go to 0 uniformly with respect to $u \in \mathcal{M}(t, T ; U)$ when $s \searrow t$, because $(\alpha+\beta)<1$.

Proposition 2.3. Let $y_{t, x, u}$ be the weak solution of (2.1). There exists a constant $C_{1}(\beta)$, independent of $u$, such that

$$
\left|y_{t, x, u}(s)-\mathrm{e}^{(s-t) A} x\right|_{X} \leq C_{1}(\beta)(s-t)^{1-\beta} \text { for all } x \in X
$$

Proof. Let $y_{t, x, u}$ be the weak solution of equation (2.1). With the integral formulation (2.14), we have

$$
\left|y_{t, x, u}(s)-\mathrm{e}^{(s-t) A} x\right|_{X} \leq\left|(-A)^{\beta} \int_{t}^{s} \mathrm{e}^{(s-r) A}[B u(r)-F(r, \Lambda y(r))] \mathrm{d} r\right|_{X} .
$$

From (2.3) and (2.10) it follows that

$$
\begin{aligned}
\left|(-A)^{\beta} \int_{t}^{s} \mathrm{e}^{(s-r) A}[B u(r)-F(r, \Lambda y(r))] \mathrm{d} r\right|_{X} & \leq \int_{t}^{s} \frac{M_{\beta}}{(s-r)^{\beta}}\left[\|B\| M_{U}+M_{F}\right] \mathrm{d} r \\
& \leq M_{\beta}\left[\|B\| M_{U}+M_{F}\right] \frac{(s-t)^{1-\beta}}{1-\beta}
\end{aligned}
$$

The proof is complete.

Proposition 2.4. We assume that $\beta=\frac{1}{2}$ and that the corresponding additional conditions of assumption (iv) is satisfied. There exists a constant $C_{1}\left(\beta_{0},|x|_{D\left((-A)^{\frac{1}{2}}\right)}\right)$, independent of $u$, such that

$$
\left|y_{t, x, u}(s)-\mathrm{e}^{(s-t) A} x\right|_{X} \leq C_{1}\left(\beta_{0},|x|_{D\left((-A)^{\frac{1}{2}}\right)}\right)(s-t)^{\frac{1}{2}+\beta_{0}} \text { for all } x \in D\left((-A)^{\frac{1}{2}}\right) .
$$


Proof. Assume that $x \in D\left((-A)^{\frac{1}{2}}\right)$. Let $y_{t, x, u}$ be the weak solution of equation (2.1). By using the integral formulation (2.14), we have

$$
\left|y_{t, x, u}(s)-\mathrm{e}^{(s-t) A} x\right|_{X} \leq\left|(-A)^{\beta-\beta_{0}} \int_{t}^{s} \mathrm{e}^{(s-r) A}\left[(-A)^{\beta_{0}} B u(r)-(-A)^{\beta_{0}} F(r, \Lambda y(r))\right] \mathrm{d} r\right|_{X} .
$$

With (2.5) and (2.10) we have

$$
\begin{aligned}
\mid(-A)^{\beta-\beta_{0}} \int_{t}^{s} \mathrm{e}^{(s-r) A}[ & {\left.\left[(-A)^{\beta_{0}} B u(r)-(-A)^{\beta_{0}} F(r, \Lambda y(r))\right] \mathrm{d} r\right|_{X} } \\
& \leq \int_{t}^{s} \frac{M_{\beta-\beta_{0}}}{(s-r)^{\beta-\beta_{0}}}\left[\left\|(-A)^{\beta_{0}} B\right\| M_{U}+M\left(\beta_{0},|x|_{D\left((-A)^{\frac{1}{2}}\right)}\right)\right] \mathrm{d} r \\
& \leq M_{\beta-\beta_{0}}\left[\left\|(-A)^{\beta_{0}} B\right\| M_{U}+M\left(\beta_{0},|x|_{D\left((-A)^{\frac{1}{2}}\right)}\right)\right] \frac{(s-t)^{1-\beta+\beta_{0}}}{1-\beta+\beta_{0}} .
\end{aligned}
$$

The proof is complete.

To prove the other propositions, we need the following theorem.

Theorem 2.5 [1] Th. 3.3.1, Chap. 2). Let $\delta$ and $\gamma$ be in $[0,1[$ and $\epsilon>0$. If the mapping

$$
t \in J \subset \mathbb{R}^{+} \mapsto t^{\delta} u(t),
$$

belongs to $L_{l o c}^{\infty}(J ; \mathbb{R})$, and if there exist two positive constants $a, b$ such that

$$
u(t) \leq a t^{-\delta}+b \int_{0}^{t}(t-\tau)^{-\gamma} u(\tau) \mathrm{d} \tau, \text { for } \quad \text { a.e. } t \in J^{*}=J \backslash\{0\},
$$

then there exits a positive constant $c:=c(\delta, \gamma, \epsilon)$ independent of $a$ and $b$ such that

$$
u(t) \leq a t^{-\delta}\left(1+c b t^{1-\gamma} \mathrm{e}^{(1+\epsilon) k(\gamma, b) t}\right) \text { for a.e. } t \in J^{*},
$$

where $k(\gamma, b):=(\Gamma(1-\gamma) b)^{1 /(1-\gamma)}$.

Proposition 2.6. Let $x$ and $x_{0}$ be in $X, u \in \mathcal{M}(t, T ; U)$, and let $y_{t, x, u}$ and $y_{t, x_{0}, u}$ be the corresponding solutions to equation (2.1). Then, for all $\theta \in\left[0,1-\alpha\left[\right.\right.$, there exists a constant $C_{2}(\alpha, \beta, \theta)$ such that:

$$
\left|\Lambda y_{t, x, u}(r)-\Lambda y_{t, x_{0}, u}(r)\right|_{X_{0}} \leq \frac{C_{2}(\alpha, \beta, \theta)}{(r-t)^{\alpha+\theta}}\left|(-A)^{-\theta}\left(x-x_{0}\right)\right|_{X}
$$

for all $r \in(t, T]$. (The constant $C_{2}(\alpha, \beta, \theta)$ is explicitly given in (2.24).)

Proof. Using the integral formulation (2.14) for $y_{t, x, u}$ and $y_{t, x_{0}, u}$, and (2.2), we obtain

$$
\begin{aligned}
\left|\Lambda y_{t, x, u}(r)-\Lambda y_{t, x_{0}, u}(r)\right|_{X_{0}} \leq & C_{\alpha}\left|(-A)^{\alpha+\theta} \mathrm{e}^{(r-t) A}(-A)^{-\theta}\left(x-x_{0}\right)\right|_{X} \\
& +C_{\alpha}\left|(-A)^{\beta+\alpha} \int_{t}^{r} \mathrm{e}^{(r-s) A}\left[F\left(s, \Lambda y_{t, x, u}(s)\right)-F\left(s, \Lambda y_{t, x_{0}, u}(s)\right)\right] \mathrm{d} s\right|_{X} .
\end{aligned}
$$

Setting $\theta=0$ in this estimate, we first obtain

$$
\left|\Lambda y_{t, x, u}(r)-\Lambda y_{t, x_{0}, u}(r)\right|_{X_{0}} \leq \frac{C_{\alpha} M_{\alpha}}{(r-t)^{\alpha}}\left|x-x_{0}\right|_{X}+\frac{2 C_{\alpha} M_{\alpha+\beta} M_{F}}{1-(\alpha+\beta)}(r-t)^{1-(\alpha+\beta)} .
$$


Multiplying both sides by $(r-t)^{\alpha+\theta}$ we have

$$
\begin{aligned}
& (r-t)^{\alpha+\theta}\left|\Lambda y_{t, x, u}(r)-\Lambda y_{t, x_{0}, u}(r)\right|_{X_{0}} \\
& \quad \leq(T-t)^{\theta} C_{\alpha} M_{\alpha}\left|x-x_{0}\right|_{X}+\frac{2 C_{\alpha} M_{\alpha+\beta} M_{F}}{1-(\alpha+\beta)}(T-t)^{1+\theta-\beta} \in L^{\infty}(t, T ; \mathbb{R})
\end{aligned}
$$

Next with (2.22) we write

$$
\begin{aligned}
& \left|\Lambda y_{t, x, u}(r)-\Lambda y_{t, x_{0}, u}(r)\right|_{X_{0}} \\
& \quad \leq \frac{C_{\alpha} M_{\alpha+\theta}}{(r-t)^{\alpha+\theta}}\left|(-A)^{-\theta}\left(x-x_{0}\right)\right|_{X}+\int_{t}^{r} \frac{C_{\alpha} M_{\beta+\alpha}}{(r-s)^{\beta+\alpha}} K_{F}\left|\Lambda y_{t, x, u}(s)-\Lambda y_{t, x_{0}, u}(s)\right|_{X_{0}} \mathrm{~d} s .
\end{aligned}
$$

Since the function $r \mapsto(r-t)^{\alpha+\theta}\left|\Lambda y_{t, x, u}(r)-\Lambda y_{t, x_{0}, u}(r)\right|_{X_{0}}$ belongs to $L^{\infty}(t, T)$, we can use Theorem 2.5 with for example $\varepsilon=1$, and we obtain $(2.21)$ by setting

$$
C_{2}(\alpha, \beta, \theta)=C_{\alpha} M_{\alpha+\theta}\left(1+C_{\beta+\alpha, \alpha+\theta}\left(c C_{\alpha} M_{\beta} K_{F}\right) T^{1-\beta+\alpha} \mathrm{e}^{2 k\left(\beta+\alpha, K_{F} C_{\alpha} M_{\beta+\alpha}\right) T}\right)
$$

where $c$ is the constant appearing in Theorem 2.5.

Proposition 2.7. Let $x$ and $x_{0}$ be in $X, u \in \mathcal{M}(t, T ; U)$, and let $y_{t, x, u}$ and $y_{t, x_{0}, u}$ be the corresponding solutions to equation (2.1). Then, for all $\theta \in\left[0,1-\alpha\left[\right.\right.$, there exists a constant $C_{3}(\alpha, \beta, \theta)$ such that

$$
\left|y_{t, x, u}(r)-y_{t, x_{0}, u}(r)\right|_{X} \leq\left(\frac{M_{\theta}}{(r-t)^{\theta}}+\frac{C_{3}(\alpha, \beta, \theta)}{1-(\alpha+\theta)}(r-t)^{1-(\alpha+\beta+\theta)}\right)\left|(-A)^{-\theta}\left(x-x_{0}\right)\right|_{X} .
$$

(The constant $C_{3}(\alpha, \beta, \theta)$ is explicitly given in $(2.27)$.)

Proof. With Proposition 2.6, we have:

$$
\begin{array}{r}
\left|y_{t, x, u}(r)-y_{t, x_{0}, u}(r)\right|_{X} \leq\left|(-A)^{\theta} \mathrm{e}^{(r-t) A}(-A)^{-\theta}\left(x-x_{0}\right)\right|_{X}+\int_{t}^{r} \frac{M_{\beta}}{(r-s)^{\beta}} K_{F}\left|\Lambda y_{t, x, u}(s)-\Lambda y_{t, x_{0}, u}(s)\right|_{X_{0}} \mathrm{~d} s \\
\leq \frac{M_{\theta}}{(r-t)^{\theta}}\left|(-A)^{-\theta}\left(x-x_{0}\right)\right|_{X}+M_{\beta} K_{F} C_{2}(\alpha, \beta, \theta)\left|(-A)^{-\theta}\left(x-x_{0}\right)\right|_{X} \int_{t}^{r} \frac{1}{(r-s)^{\beta}} \frac{1}{(s-t)^{\alpha+\theta}} \mathrm{d} s \\
\quad \leq\left(\frac{M_{\theta}}{(r-t)^{\theta}}+M_{\beta} K_{F} C_{2}(\alpha, \beta, \theta) \int_{t}^{r} \frac{1}{(r-s)^{\beta}} \frac{1}{(s-t)^{\alpha+\theta}} \mathrm{d} s\right)\left|(-A)^{-\theta}\left(x-x_{0}\right)\right|_{X} .
\end{array}
$$

By using the integral formula of the beta function we have:

$$
\int_{t}^{r} \frac{1}{(r-s)^{\beta}(s-t)^{\alpha+\theta}} \mathrm{d} s=\frac{\Gamma(1-\beta) \Gamma(1-(\alpha+\theta))}{\Gamma(2-(\alpha+\theta+\beta))}(r-t)^{1-(\alpha+\theta+\beta)} .
$$

By setting

and

$$
C_{3}(\alpha, \beta, \theta)=\frac{\Gamma(1-\beta) \Gamma(1-(\alpha+\theta))}{\Gamma(2-(\alpha+\theta+\beta))} M_{\beta} K_{F} C_{2}(\alpha, \beta, \theta)
$$

$$
C_{4}(\alpha, \beta, \theta, t ; r)=\left(\frac{M_{\theta}}{(r-t)^{\theta}}+\frac{C_{3}(\alpha, \beta, \theta)}{1-(\alpha+\theta)}(r-t)^{1-(\alpha+\beta+\theta)}\right)
$$


we obtain

$$
\left|y_{t, x, u}(r)-y_{t, x_{0}, u}(r)\right|_{X} \leq C_{4}(\alpha, \beta, \theta, t ; r)\left|(-A)^{-\theta}\left(x-x_{0}\right)\right|_{X} .
$$

Proposition 2.8. Let $x$ be in $X, s, t \in[0, T)$, and $u \in \mathcal{M}(\min (t, s), T ; U)$. Let us denote by $y_{t, x, u}$ and $y_{s, x, u}$ the solutions of equation (2.1) respectively corresponding to the initial data $(t, x)$ and $(s, x)$. Then there exist a constant $C_{5}(\alpha, \beta)$ and a continuous mapping $\bar{a}(t, s, x)$ (independent of $\alpha$ ) such that, for all $\left.\left.r \in\right] \max (s, t), T\right]$, we have:

$$
\left|\Lambda y_{t, x, u}(r)-\Lambda y_{s, x, u}(r)\right|_{X_{0}} \leq \frac{C_{5}(\alpha, \beta)}{(r-\max (t, s))^{\alpha}} \bar{a}(t, s, x) .
$$

The function $s \mapsto \bar{a}(t, s, x)$ goes to 0 when $s$ goes to $t$, for all fixed $x \in X$. (The constant $C_{5}(\alpha, \beta)$ and the mapping $\bar{a}$ are explicitly defined in (2.34) and (2.35).)

Proposition 2.9. With the same assumptions and notation as in the previous proposition, there exists a constant $C_{6}(\alpha, \beta)$ such that, for all $r \in[\max (s, t), T]$, we have:

$$
\left|y_{t, x, u}(r)-y_{s, x, u}(r)\right|_{X} \leq C_{6}(\alpha, \beta) \bar{a}(t, s, x) .
$$

(The constant $C_{6}(\alpha, \beta)$ is explicitly defined in (2.36).)

Proof of Proposition 2.8. Consider the case where $s<t$. The case $t<s$ can be treated in a similar way. Let be $r>t>s$, with estimate (2.2) and with (2.14), we have

$$
\begin{aligned}
\left|\Lambda y_{t, x, u}(r)-\Lambda y_{s, x, u}(r)\right|_{X_{0}} \leq & C_{\alpha}\left|(-A)^{\alpha} \mathrm{e}^{(r-t) A}\left(\mathrm{e}^{|t-s| A} x-x\right)\right|_{X} \\
& +C_{\alpha}\left|\int_{s}^{t}(-A)^{\alpha+\beta} \mathrm{e}^{(r-\sigma) A}\left[B u(\sigma)-F\left(\sigma, \Lambda y_{s, x, u}(\sigma)\right)\right] \mathrm{d} \sigma\right|_{X} \\
& +C_{\alpha}\left|\int_{t}^{r}(-A)^{\alpha+\beta} \mathrm{e}^{(r-\sigma) A}\left[F\left(\sigma, \Lambda y_{t, x, u}(\sigma)\right)-F\left(\sigma, \Lambda y_{s, x, u}(\sigma)\right)\right] \mathrm{d} \sigma\right|_{X} .
\end{aligned}
$$

Now we can write

$$
(2.31) \leq \frac{C_{\alpha} M_{\alpha}}{(r-t)^{\alpha}}\left|\left(\mathrm{e}^{|t-s| A}-I\right) x\right|_{X} .
$$

Since $(r-t)^{\alpha} \leq(r-\sigma)^{\alpha}$ for all $\sigma \in(s, t)$, we have

$$
(2.32) \leq C_{\alpha} M_{\alpha+\beta}\left(M_{F}+\|B\| M_{U}\right) \int_{s}^{t} \frac{\mathrm{d} \sigma}{(r-\sigma)^{\alpha+\beta}} \leq \frac{C_{\alpha} M_{\alpha+\beta}\left(M_{F}+\|B\| M_{U}\right)}{(r-t)^{\alpha}} \int_{s}^{t} \frac{\mathrm{d} \sigma}{(r-\sigma)^{\beta}} .
$$

Similarly, for all $\sigma \in(s, t)$, we have $(r-\sigma)^{\beta} \geq(t-\sigma)^{\beta}$. Then

$$
\int_{s}^{t} \frac{\mathrm{d} \sigma}{(r-\sigma)^{\beta}} \leq \int_{s}^{t} \frac{\mathrm{d} \sigma}{(t-\sigma)^{\beta}}=\frac{(t-s)^{1-\beta}}{1-\beta}
$$

and therefore we obtain

The last term can be estimated as follows

$$
(2.32) \leq \frac{C_{\alpha} M_{\alpha+\beta}\left(M_{F}+\|B\| M_{U}\right)}{(r-t)^{\alpha}(1-\beta)}|t-s|^{1-\beta} .
$$

$$
\begin{aligned}
(2.33) & \leq \int_{t}^{r} \frac{C_{\alpha} M_{\alpha+\beta} K_{F}}{(r-\sigma)^{\alpha+\beta}}\left|\Lambda y_{t, x, u}(\sigma)-\Lambda y_{s, x, u}(\sigma)\right|_{X_{0}} \mathrm{~d} \sigma \\
& \leq \frac{C_{\alpha} M_{\alpha+\beta} K_{F}}{(r-t)^{\alpha}} \int_{t}^{r} \frac{1}{(r-\sigma)^{\beta}}\left|\Lambda y_{t, x, u}(\sigma)-\Lambda y_{s, x, u}(\sigma)\right|_{X_{0}} \mathrm{~d} \sigma .
\end{aligned}
$$


From the estimates obtained for (2.31), (2.32), (2.33), we deduce that the function $r \mapsto$ $(r-t)^{\alpha}\left|\Lambda y_{t, x, u}(r)-\Lambda y_{s, x, u}(r)\right|_{X_{0}}$ belongs to $L^{\infty}(t, T)$. Applying Theorem 2.5, we obtain

$$
\left|\Lambda y_{t, x, u}(r)-\Lambda y_{s, x, u}(r)\right|_{X_{0}} \leq \frac{C_{5}(\alpha, \beta)}{(r-t)^{\alpha}} \bar{a}(t, s, x),
$$

with

$$
C_{5}(\alpha, \beta)=2 C_{\alpha} \max \left(\frac{M_{\alpha}}{1-\alpha}, \frac{M_{\alpha+\beta}\left(M_{F}+\|B\| M_{U}\right)}{1-\beta}\right)\left(1+c C_{\alpha} M_{\beta+\alpha} K_{F} T^{(1-\beta)} \mathrm{e}^{C T}\right),
$$

where $c$ and $C$ are given in Theorem 2.5, and

$$
\bar{a}(t, s, x)=\left|\mathrm{e}^{|t-s| A} x-x\right|_{X}+|t-s|^{1-\beta} .
$$

The function $s \mapsto \bar{a}(t, s, x)$ goes to 0 when $s$ goes to $t$, for all $x$ fixed in $X$.

Proof of Proposition 2.9. Consider the case where $0 \leq s<t$. We have:

$$
\begin{aligned}
\mid y_{t, x, u}(r) & -\left.y_{s, x, u}(r)\right|_{X} \\
& \leq\left|\mathrm{e}^{|t-s| A} x-x\right|_{X}+\frac{M_{\beta}\left(M_{F}+\|B\| M_{U}\right)}{1-\beta}(t-s)^{1-\beta}+C_{5}(\alpha, \beta) K_{F} \bar{a}(t, s, x) \int_{t}^{r} \frac{M_{\beta}}{(r-\sigma)^{\beta}} \frac{1}{(\sigma-t)^{\alpha}} \mathrm{d} \sigma .
\end{aligned}
$$

From (2.26) with $\theta=0$, it yields:

$$
\int_{t}^{r} \frac{1}{(r-\sigma)^{\beta}} \frac{1}{(\sigma-t)^{\alpha}} \mathrm{d} \sigma \leq \frac{\Gamma(1-\beta) \Gamma(1-\alpha)}{\Gamma(2-(\alpha+\beta))} T^{1-(\alpha+\beta)} \leq 4 T^{1-(\alpha+\beta)}
$$

Hence

$$
\left|y_{t, x, u}(r)-y_{s, x, u}(r)\right|_{X} \leq\left(1+\frac{M_{\beta}\left(M_{F}+\|B\| M_{U}\right)}{1-\beta}+C_{5}(\alpha, \beta) K_{F} 4 T^{1-(\alpha+\beta)}\right) \bar{a}(t, s, x) .
$$

The proof is complete.

Proposition 2.10. Let $x$ and $x_{0}$ be in $X, t \in[0, T)$, and $u \in \mathcal{M}(t, T ; U)$. Let us denote by $y_{t, x, u}$ and $y_{t, x_{0}, u}$ the solutions of equation (2.1) respectively corresponding to the initial data $(t, x)$ and $\left(t, x_{0}\right)$. Then, for all $r \in[t, T]$ and all $s \in] t, T]$, we have:

$$
\left|\Lambda y_{t, x, u}(s)-\Lambda y_{t, x_{0}, u}(s)\right|_{X_{0}} \leq \frac{C_{7}(\alpha, \beta)}{(s-t)^{\alpha}}\left|x-x_{0}\right|_{X}
$$

and

$$
\left|y_{t, x, u}(r)-y_{t, x_{0}, u}(r)\right|_{X} \leq C_{8}(\alpha, \beta)\left|x-x_{0}\right|_{X} .
$$

Proof. The function $w=y_{t, x, u}-y_{t, x_{0}, u}$ satisfies

$$
\begin{aligned}
|\Lambda w(s)|_{X_{0}} & \leq \frac{M_{\alpha}}{(s-t)^{\alpha}}\left|x-x_{0}\right|_{X}+\left|(-A)^{\beta+\alpha} \int_{t}^{s} \mathrm{e}^{(s-r) A}\left[F\left(r, \Lambda y_{t, x, u}(r)\right)-F\left(r, \Lambda y_{, x_{0}, u}(r)\right)\right] \mathrm{d} r\right|_{X} \\
& \leq \frac{M_{\alpha}}{(s-t)^{\alpha}}\left|x-x_{0}\right|_{X}+M_{\alpha+\beta} K_{F} \int_{t}^{s} \frac{1}{(s-r)^{\alpha+\beta}}|\Lambda w(r)|_{X_{0}} \mathrm{~d} r .
\end{aligned}
$$


Estimate (2.37) now follows from Theorem 2.5. We can also obtain the estimate

$$
|w(r)|_{X} \leq\left|x-x_{0}\right|_{X}+K_{F} \int_{t}^{r} \frac{1}{(r-s)^{\beta}} \frac{C_{\alpha}}{(s-t)^{\alpha}}\left|x-x_{0}\right|_{X} \mathrm{~d} s .
$$

By the same calculation as in the proof of Proposition 2.7, we have

$$
|w(r)|_{X} \leq\left[1+K_{F} C_{\alpha} T^{1-(\alpha+\beta)}\right]\left|x-x_{0}\right|_{X}
$$

and (2.38) is established.

\section{Viscosity solutions AND UniQUEnESS RESUlt}

In this section we study the uniqueness of solution to equation (1.1). It is well known that, by a change of variable in time, the terminal value problem (1.1) is equivalent the Hamilton-Jacobi-Bellman equation

$$
\begin{aligned}
& \frac{\partial v}{\partial t}(t, x)-\left(D_{x} v(t, x) \mid A x\right)_{X}+\left((-A)^{\beta} D_{x} v(t, x) \mid F(t, \Lambda x)\right)_{X} \\
& \left.\left.v(0, x)=g(x) . \quad+H\left(t, x,(-A)^{\beta} D_{x} v(t, x)\right)=0, \quad \forall(t, x) \in\right] 0, T\right] \times X,
\end{aligned}
$$

Let $C_{A}^{1}(] 0, T[\times X)$ be the set of all functions $\Phi$ (called test functions) satisfying the following conditions:

( $\alpha) \Phi \in C^{1}(] 0, T[\times X)$.

( $\beta$ ) $D_{x} \Phi(\cdot, x)$ is constant (in $\left.t\right)$ and $D_{x} \Phi(t, \cdot)$ is Lipschitz on $X$, i.e.:

$$
\left|D_{x} \Phi(t, x)-D_{x} \Phi(t, y)\right|_{X} \leq K_{\Phi}|x-y|_{X} .
$$

( $\gamma$ ) For all $\theta \in\left[0,1-\alpha\left[, D_{x} \Phi(t, x)\right.\right.$ belongs to $D\left((-A)^{\theta}\right)$ if and only if $x \in D\left((-A)^{\theta}\right)$.

$(\delta)$ The mapping $x \mapsto D_{x} \Phi(t, x)$ is continuous from $D\left((-A)^{\frac{1}{2}}\right)$ into itself.

Remark 3.1. Since $D_{x} \Phi(t, x)$ does not depend on $t$, from the last condition we can infer that the mapping $(t, x) \mapsto D_{x} \Phi(t, x)$ is continuous from $[0, T] \times D\left((-A)^{\frac{1}{2}}\right)$ into $D\left((-A)^{\frac{1}{2}}\right)$.

Definition 3.2. Consider functions $w$ satisfying:

(i) $w \in C([0, T] \times X)$ and $|w(t, x)| \leq M_{w}$, for all $(t, x) \in[0, T] \times X$.

(ii) $|w(t, x)-w(t, y)| \leq K_{w}|x-y|_{X}$ for all $t \in[0, T]$, and all $x, y \in X$.

(iii) $|w(t, x)-w(t, y)| \leq C_{t, \theta}\left|(-A)^{-\theta}(x-y)\right|_{X}$, for all $\left.\left.t \in\right] 0, T\right]$, all $x, y \in X$, and all $\theta \in[0,1-\alpha[$, where the constant $C_{t, \theta}$ is bounded on all compact subset of $\left.] 0, T\right]$.

(iv) $w(\cdot, x)$ is Hölder continuous in time of exponent $0<\eta \leq 1$, for all $x \in D\left((-A)^{\frac{1}{2}}\right)$. More precisely there exists a constant $M_{1, w}$ such that:

$$
|w(t, x)-w(s, x)| \leq M_{1, w}\left(1+\left|(-A)^{\frac{1}{2}} x\right|_{X}\right)|t-s|^{\eta} .
$$

We say that a function $w$ satisfying (i)-(iv) is a viscosity subsolution of (3.1) on $[0, T]$ if, for every $\Phi \in$ $C_{A}^{1}(] 0, T[\times X)$, the conditions $\left(\alpha_{1}\right)$ and $\left(\beta_{1}\right)$ are satisfied, where:

$\left(\alpha_{1}\right)$

$$
\frac{\partial \Phi}{\partial t}(t, x)+H\left(t, x,(-A)^{\beta} D_{x} \Phi(t, x)\right)
$$

$$
+\left((-A)^{\frac{1}{2}} D_{x} \Phi(t, x) \mid(-A)^{\frac{1}{2}} x\right)_{X}+\left((-A)^{\beta} D_{x} \Phi(t, x) \mid F(t, \Lambda x)\right)_{X} \leq 0
$$

for all $(t, x) \in(] 0, T\left[\times D\left((-A)^{\frac{1}{2}}\right)\right) \cap \arg \max (w-\Phi)$, 


$$
\lim _{t \searrow 0} \sup _{x \in X}\left[w(t, x)-g\left(\mathrm{e}^{t A} x\right)\right]^{+}=0 .
$$

(Recall that $[f]^{+}=\max (f, 0)$ and $\left.[f]^{-}=\min (f, 0).\right)$

We say that a function $w$ satisfying (i)-(iv) is a viscosity supersolution of (3.1) on $[0, T]$ if, for every $\Phi \in$ $C_{A}^{1}(] 0, T[\times X)$, the two conditions $\left(\alpha_{2}\right)$ and $\left(\beta_{2}\right)$ are satisfied, where:

$\left(\alpha_{2}\right)$

$$
\frac{\partial \Phi}{\partial t}(t, x)+H\left(t, x,(-A)^{\beta} D_{x} \Phi(t, x)\right)
$$

$$
+\left((-A)^{\frac{1}{2}} D_{x} \Phi(t, x) \mid(-A)^{\frac{1}{2}} x\right)_{X}+\left((-A)^{\beta} D_{x} \Phi(t, x) \mid F(t, \Lambda x)\right)_{X} \geq 0
$$

for all $(t, x) \in(] 0, T\left[\times D\left((-A)^{\frac{1}{2}}\right)\right) \cap \arg \min (w-\Phi)$,

$$
\lim _{t \backslash 0} \sup _{x \in X}\left[w(t, x)-g\left(\mathrm{e}^{t A} x\right)\right]^{-}=0
$$

Finally, $w$ is a viscosity solution of (3.1) if it is both a subsolution and a supersolution of equation (3.1).

Remark 3.3. If $(t, x)$ belongs to $] 0, T\left[\times D\left((-A)^{\frac{1}{2}}\right)\right.$, then $\Lambda x$ is well defined and $F(t, \Lambda x)$ is meaningful.

Remark 3.4. If in place of equation (3.1) we consider equation (1.1), the conditions $\left(\alpha_{1}\right),\left(\alpha_{2}\right),\left(\beta_{1}\right)$, and $\left(\beta_{2}\right)$ have to be modified accordingly (see Sect. 4).

Theorem 3.5. Assume that (i) - (vii) of Section 2 hold. Let $w$ be a viscosity subsolution and $v$ be a viscosity supersolution of the Hamilton-Jacobi-Bellman equation (3.1). Then

$$
w(t, x) \leq v(t, x) \quad \text { for all }(t, x) \in[0, T] \times X .
$$

Before proving this theorem let us state a useful lemma.

Lemma 3.6. Assume that $\varphi$ and $\psi \in C_{A}^{1}(] 0, T[\times X)$, and let $w$ and $v$ be two continuous functions in $[0, T] \times$ $X$. If

$$
\left(t_{0}, x_{0}\right) \in \operatorname{argmax}_{\mathcal{O}}(w-\varphi) \quad \text { and } \quad\left(s_{0}, y_{0}\right) \in \operatorname{argmin}_{\mathcal{O}}(v-\psi)
$$

where $\mathcal{O}$ is an open set of $] 0, T[\times X$, then

$$
D_{x} \varphi\left(t_{0}, x_{0}\right) \subset D_{x}^{+} w\left(t_{0}, x_{0}\right) \text { and } D_{x} \psi\left(s_{0}, y_{0}\right) \subset D_{x}^{-} v\left(s_{0}, y_{0}\right) .
$$

Proof. We establish the result only for the function $\varphi$. Due to (3.3), for all $x \in X$, we have:

$$
w\left(t_{0}, x\right)-w\left(t_{0}, x_{0}\right)-\left[\varphi\left(t_{0}, x\right)-\varphi\left(t_{0}, x_{0}\right)\right] \leq 0 .
$$

With the condition $(\beta)$ in the definition of $C_{A}^{1}(] 0, T[\times X)$, we have:

$$
\left|\varphi\left(t_{0}, x\right)-\varphi\left(t_{0}, x_{0}\right)-\left(D_{x} \varphi\left(t_{0}, x_{0}\right) \mid x-x_{0}\right)_{X}\right| \leq K_{\Phi}\left|x-x_{0}\right|^{2} .
$$

Combining this estimate with the previous inequality, we obtain:

$$
\limsup _{\left|x-x_{0}\right| \rightarrow 0} \frac{w\left(t_{0}, x\right)-w\left(t_{0}, x_{0}\right)-\left(D_{x} \varphi\left(t_{0}, x_{0}\right) \mid x-x_{0}\right)_{X}}{\left|\left(x-x_{0}\right)\right|} \leq 0 .
$$

Let us recall Young's inequality. For all $p, q>1$ such that $\frac{1}{p}+\frac{1}{q}=1$, we have:

$$
a b \leq \frac{\lambda^{p}}{p} a^{p}+\frac{1}{q \lambda^{q}} b^{q} \text { for all } \lambda>0, \text { and all } a, b \geq 0 .
$$


Proof of Theorem 3.5. We are going to use the same kind of proof as in [6]. The proof is divided in five steps.

Step 1. Since $w, v \in C([0, T] \times X)$, it is enough to prove that

$$
\left.w_{\sigma}(t, x) \leq v_{\sigma}(t, x) \text { for all }(t, x) \in\right] 0, T[\times X \text { and all } \sigma>0,
$$

where

$$
w_{\sigma}(t, x)=w(t, x)-\frac{\sigma}{T-t} \text { and } v_{\sigma}(t, x)=v(t, x)+\frac{\sigma}{T-t} .
$$

As $w$ is a subsolution of (3.1), then $w_{\sigma}$ is a subsolution of

$$
\begin{gathered}
\frac{\partial w_{\sigma}}{\partial t}(t, x)-\left(D_{x} w_{\sigma}(t, x) \mid A x\right)_{X}+\left((-A)^{\beta} D_{x} w_{\sigma}(t, x) \mid F(t, \Lambda x)\right)_{X} \\
+H\left(t, x,(-A)^{\beta} D_{x} w_{\sigma}(t, x)\right)=-\frac{\sigma}{(T-t)^{2}} \leq-\frac{\sigma}{T^{2}}, \\
w_{\sigma}(0, x)=g(x)-\frac{\sigma}{T} .
\end{gathered}
$$

Similarly, $v_{\sigma}$ is a supersolution of

$$
\begin{aligned}
\frac{\partial v_{\sigma}}{\partial t}(t, x) & -\left(D_{x} v_{\sigma}(t, x) \mid A x\right)_{X}+\left((-A)^{\beta} D_{x} v_{\sigma}(t, x) \mid F(t, \Lambda x)\right)_{X} \\
& +H\left(t, x,(-A)^{\beta} D_{x} v_{\sigma}(t, x)\right)=\frac{\sigma}{(T-t)^{2}} \geq \frac{\sigma}{T^{2}}, \\
v_{\sigma}(0, x)= & g(x)+\frac{\sigma}{T} .
\end{aligned}
$$

Step 2. Let $0<\eta \leq 1$ be an exponent such that $v(\cdot, x)$ and $w(\cdot, x)$ be Hölder continuous of exponent $\eta$ (Condition (iv) in Def. 3.2). We set

$$
\bar{\eta}=\min \left(\eta, \eta_{1}, \eta_{2}\right)
$$

Let $\varepsilon$ and $\mu$ be in $] 0,1]$. For all $(t, x)$ and all $(s, y)$ in $] 0, T[\times X$, we define

$$
\Phi_{\varepsilon, \mu}(t, s, x, y)=w_{\sigma}(t, x)-v_{\sigma}(s, y)-\frac{1}{2 \varepsilon}\left((-A)^{-1}(x-y) \mid(x-y)\right)_{X}-\frac{(t-s)^{2}}{2 \varepsilon^{\frac{2}{\eta}}}-\frac{\mu}{2}\left(|x|_{X}^{2}+|y|_{X}^{2}\right) .
$$

Let $\tau$ be a small parameter satisfying $0<\tau<\frac{T}{2}$, and set $Q_{\tau}=[\tau, T-\tau] \times X$. From condition (iii) in Definition 3.2, $v$ and $w$ are weakly continuous in $Q_{\tau}$. Thus $v_{\sigma}$ and $w_{\sigma}$ are weakly continuous in $Q_{\tau}$. Moreover the mapping

$$
(x, y) \mapsto \frac{1}{2 \varepsilon}\left((-A)^{-1}(x-y) \mid(x-y)\right)_{X}+\frac{\mu}{2}|x|_{X}^{2}+\frac{\mu}{2}|y|_{X}^{2}
$$

is convex, and continuous (for the strong topology of $X \times X$ ). Therefore $\Phi_{\varepsilon, \mu}$ is weakly lower semicontinuous in $Q_{\tau}^{2}$. Besides for all couples $(t, x),(s, y) \in Q_{\tau}$

$$
\Phi_{\varepsilon, \mu}(t, s, x, y) \leq M_{w}+M_{v}-\frac{\mu}{2}\left(|x|_{X}^{2}+|y|_{X}^{2}\right) \rightarrow-\infty \text { when } \max \left(|x|_{X},|y|_{X}\right) \rightarrow+\infty .
$$

Thus there exists $\left(t_{\varepsilon, \mu}, x_{\varepsilon, \mu}, s_{\varepsilon, \mu}, y_{\varepsilon, \mu}\right) \in Q_{\tau}^{2}$ such that

$$
\Phi_{\varepsilon, \mu}\left(t_{\varepsilon, \mu}, s_{\varepsilon, \mu}, x_{\varepsilon, \mu}, y_{\varepsilon, \mu}\right)=\max _{Q_{\tau} \times Q_{\tau}} \Phi_{\varepsilon, \mu} .
$$

Let us verify that if $\tau$ is small enough $\left(0<\tau<\tau_{\sigma}\right)$, then $t_{\varepsilon, \mu}, s_{\varepsilon, \mu}<T-\tau$. Indeed, from the inequality

$$
w_{\sigma}(t, x)-v_{\sigma}(s, y) \leq M_{w}+M_{v}-\frac{\sigma}{T-t}-\frac{\sigma}{T-s},
$$


it follows that

$$
\lim _{\max (s, t) \rightarrow T}\left(w_{\sigma}(t, x)-v_{\sigma}(s, y)\right)=-\infty \quad \text { uniformly w.r. to } x \text { and } y .
$$

Hence we have shown that there exists $\left(t_{\varepsilon, \mu}, x_{\varepsilon, \mu}, s_{\varepsilon, \mu}, y_{\varepsilon, \mu}\right) \in\left(\left[\tau, T-\tau[\times X)^{2}\right.\right.$ such that

$$
\Phi_{\varepsilon, \mu}\left(t_{\varepsilon, \mu}, s_{\varepsilon, \mu}, x_{\varepsilon, \mu}, y_{\varepsilon, \mu}\right)=\max _{Q_{\tau} \times Q_{\tau}} \Phi_{\varepsilon, \mu}
$$

Step 3. We are going to obtain some a priori estimates on $t_{\varepsilon, \mu}, s_{\varepsilon, \mu}, x_{\varepsilon, \mu}, y_{\varepsilon, \mu}$. Since

$$
\Phi_{\varepsilon, \mu}\left(t_{\varepsilon, \mu}, t_{\varepsilon, \mu}, x_{\varepsilon, \mu}, x_{\varepsilon, \mu}\right)+\Phi_{\varepsilon, \mu}\left(s_{\varepsilon, \mu}, s_{\varepsilon, \mu}, y_{\varepsilon, \mu}, y_{\varepsilon, \mu}\right) \leq 2 \Phi_{\varepsilon, \mu}\left(t_{\varepsilon, \mu}, s_{\varepsilon, \mu}, x_{\varepsilon, \mu}, y_{\varepsilon, \mu}\right),
$$

then

$$
\begin{aligned}
w_{\sigma}\left(t_{\varepsilon, \mu}, x_{\varepsilon, \mu}\right)- & v_{\sigma}\left(t_{\varepsilon, \mu}, x_{\varepsilon, \mu}\right)+w_{\sigma}\left(s_{\varepsilon, \mu}, y_{\varepsilon, \mu}\right)-v_{\sigma}\left(s_{\varepsilon, \mu}, y_{\varepsilon, \mu}\right) \\
& \leq 2\left(w_{\sigma}\left(t_{\varepsilon, \mu}, x_{\varepsilon, \mu}\right)-v_{\sigma}\left(s_{\varepsilon, \mu}, y_{\varepsilon, \mu}\right)\right)-\frac{1}{\varepsilon}\left((-A)^{-1}\left(x_{\varepsilon, \mu}-y_{\varepsilon, \mu}\right) \mid\left(x_{\varepsilon, \mu}-y_{\varepsilon, \mu}\right)\right)_{X}-\frac{\left(t_{\varepsilon, \mu}-s_{\varepsilon, \mu}\right)^{2}}{\varepsilon^{\frac{2}{\eta}}} .
\end{aligned}
$$

Consequently we obtain

$$
\begin{aligned}
\frac{1}{\varepsilon}\left((-A)^{-1}\left(x_{\varepsilon, \mu}-y_{\varepsilon, \mu}\right)\right. & \left.\mid\left(x_{\varepsilon, \mu}-y_{\varepsilon, \mu}\right)\right)_{X}+\frac{\left(t_{\varepsilon, \mu}-s_{\varepsilon, \mu}\right)^{2}}{\varepsilon^{\frac{2}{\eta}}} \\
\leq & w_{\sigma}\left(t_{\varepsilon, \mu}, x_{\varepsilon, \mu}\right)-w_{\sigma}\left(s_{\varepsilon, \mu}, y_{\varepsilon, \mu}\right)+v_{\sigma}\left(t_{\varepsilon, \mu}, x_{\varepsilon, \mu}\right)-v_{\sigma}\left(s_{\varepsilon, \mu}, y_{\varepsilon, \mu}\right) \\
\leq & 2\left(M_{w}+M_{v}\right)=C .
\end{aligned}
$$

We deduce that

$$
\begin{aligned}
\left|t_{\varepsilon, \mu}-s_{\varepsilon, \mu}\right| & \leq C \varepsilon^{\frac{1}{\bar{\eta}}} \\
\left|(-A)^{-\frac{1}{2}}\left(x_{\varepsilon, \mu}-y_{\varepsilon, \mu}\right)\right|_{X} & \leq C \sqrt{\varepsilon}
\end{aligned}
$$

Now let us show that

$$
\lim _{\mu \searrow 0} \mu\left(\left|x_{\varepsilon(\mu), \mu}\right|_{X}^{2}+\left|y_{\varepsilon(\mu), \mu}\right|_{X}^{2}\right)=0
$$

where $0<\varepsilon(\mu)<1$ is any function of $\mu$. For all $x \in X$, we have:

$$
\Phi_{\varepsilon, \mu}\left(t_{\varepsilon, \mu}, t_{\varepsilon, \mu}, x, x\right) \leq \Phi_{\varepsilon, \mu}\left(t_{\varepsilon, \mu}, s_{\varepsilon, \mu}, x_{\varepsilon, \mu}, y_{\varepsilon, \mu}\right)
$$

i.e.

$$
\begin{gathered}
w_{\sigma}\left(t_{\varepsilon, \mu}, x\right)-v_{\sigma}\left(t_{\varepsilon, \mu}, x\right)-\mu|x|_{X}^{2} \leq \\
w_{\sigma}\left(t_{\varepsilon, \mu}, x_{\varepsilon, \mu}\right)-v_{\sigma}\left(s_{\varepsilon, \mu}, y_{\varepsilon, \mu}\right)-\frac{1}{2 \varepsilon}\left((-A)^{-1}\left(x_{\varepsilon, \mu}-y_{\varepsilon, \mu}\right) \mid\left(x_{\varepsilon, \mu}-y_{\varepsilon, \mu}\right)\right)_{X} \\
-\frac{\left(t_{\varepsilon, \mu}-s_{\varepsilon, \mu}\right)^{2}}{\varepsilon^{\frac{2}{\eta}}}-\frac{\mu}{2}\left(\left|x_{\varepsilon, \mu}\right|_{X}^{2}+\left|y_{\varepsilon, \mu}\right|_{X}^{2}\right) .
\end{gathered}
$$

By taking $x=0$, we deduce that

$$
\frac{\mu}{2}\left(\left|x_{\varepsilon, \mu}\right|_{X}^{2}+\left|y_{\varepsilon, \mu}\right|_{X}^{2}\right) \leq 2\left(M_{w}+M_{v}\right)
$$

Thus, $\frac{\mu}{2}\left(\left|x_{\varepsilon, \mu}\right|_{X}^{2}+\left|y_{\varepsilon, \mu}\right|_{X}^{2}\right)$ is bounded independently of $\left.\left.\varepsilon \in\right] 0,1\right]$ and $\left.\left.\mu \in\right] 0,1\right]$. We are going to use this property to prove (3.13). 
We define now $\Psi_{\varepsilon}$ on $Q_{\tau} \times Q_{\tau}$ by:

$$
\Psi_{\varepsilon}(t, s, x, y)=w_{\sigma}(t, x)-v_{\sigma}(s, y)+\frac{1}{2 \varepsilon}\left(A^{-1}(x-y) \mid(x-y)\right)_{X}-\frac{(t-s)^{2}}{2 \varepsilon^{\frac{2}{\eta}}} .
$$

The function $\Psi_{\varepsilon}$ is bounded from above (independently of $\varepsilon$ ):

$$
\Psi_{\varepsilon} \leq M_{w}+M_{v}
$$

and upper semicontinuous. So, for every $\delta>0$, there exists $\left(t_{\varepsilon, \delta}, x_{\varepsilon, \delta}, s_{\varepsilon, \delta}, y_{\varepsilon, \delta}\right) \in Q_{\tau}^{2}$ such that

$$
\Psi_{\varepsilon}\left(t_{\varepsilon, \delta}, s_{\varepsilon, \delta}, x_{\varepsilon, \delta}, y_{\varepsilon, \delta}\right) \geq \Psi_{\varepsilon}(t, s, x, y)-\delta \quad \forall(t, x),(s, y) \in Q_{\tau} .
$$

The point $\left(t_{\varepsilon, \delta}, s_{\varepsilon, \delta}, x_{\varepsilon, \delta}, y_{\varepsilon, \delta}\right)$ corresponds to a supremum (and a priori not a maximum), because we have not proved that $\Psi_{\varepsilon} \rightarrow-\infty$ when $\max \left(|x|_{X},|y|_{X}\right) \rightarrow \infty$. Starting from the inequality

$$
\Phi_{\varepsilon, \mu}\left(t_{\varepsilon, \mu}, s_{\varepsilon, \mu}, x_{\varepsilon, \mu}, y_{\varepsilon, \mu}\right) \geq \Phi_{\varepsilon, \mu}\left(t_{\varepsilon, \delta}, s_{\varepsilon, \delta}, x_{\varepsilon, \delta}, y_{\varepsilon, \delta}\right)
$$

it follows that

$$
\begin{aligned}
\Psi_{\varepsilon}\left(t_{\varepsilon, \mu}, s_{\varepsilon, \mu}, x_{\varepsilon, \mu}, y_{\varepsilon, \mu}\right)-\frac{\mu}{2}\left(\left|x_{\varepsilon, \mu}\right|_{X}^{2}+\left|y_{\varepsilon, \mu}\right|_{X}^{2}\right) & \geq \Psi_{\varepsilon}\left(t_{\varepsilon, \delta}, s_{\varepsilon, \delta}, x_{\varepsilon, \delta}, x_{\varepsilon, \delta}\right)-\frac{\mu}{2}\left(\left|x_{\varepsilon, \delta}\right|_{X}^{2}+\left|y_{\varepsilon, \delta}\right|_{X}^{2}\right) \\
& \geq \Psi_{\varepsilon}\left(t_{\varepsilon, \mu}, s_{\varepsilon, \mu}, x_{\varepsilon, \mu}, y_{\varepsilon, \mu}\right)-\delta-\frac{\mu}{2}\left(\left|x_{\varepsilon, \delta}\right|_{X}^{2}+\left|y_{\varepsilon, \delta}\right|_{X}^{2}\right) .
\end{aligned}
$$

Hence we have

$$
\frac{\mu}{2}\left(\left|x_{\varepsilon, \mu}\right|_{X}^{2}+\left|y_{\varepsilon, \mu}\right|_{X}^{2}\right) \leq \frac{\mu}{2}\left(\left|x_{\varepsilon, \delta}\right|_{X}^{2}+\left|y_{\varepsilon, \delta}\right|_{X}^{2}\right)+\delta \quad \text { for all } \delta>0 .
$$

This inequality is satisfied for all $\varepsilon>0$. In particular if $0<\varepsilon(\mu)<1$ is a function of $\mu$, we can write

$$
\frac{\mu}{2}\left(\left|x_{\varepsilon(\mu), \mu}\right|_{X}^{2}+\left|y_{\varepsilon(\mu), \mu}\right|_{X}^{2}\right) \leq \frac{\mu}{2}\left(\left|x_{\varepsilon(\mu), \delta}\right|_{X}^{2}+\left|y_{\varepsilon(\mu), \delta}\right|_{X}^{2}\right)+\delta \leq \mu \frac{2}{\delta}\left(M_{w}+M_{v}\right)+\delta .
$$

Thus

$$
\limsup _{\mu \searrow 0} \frac{\mu}{2}\left(\left|x_{\varepsilon(\mu), \mu}\right|_{X}^{2}+\left|y_{\varepsilon(\mu), \mu}\right|_{X}^{2}\right)=\delta
$$

We take the limit when $\delta \rightarrow 0$ to obtain (3.13).

Let us prove that

$$
\frac{1}{2 \varepsilon}\left|(-A)^{-\frac{1}{2}}\left(x_{\varepsilon, \mu}-y_{\varepsilon, \mu}\right)\right|_{X}^{2} \leq \frac{\left|x_{\varepsilon, \mu}-y_{\varepsilon, \mu}\right|_{X}^{2}}{8 \varepsilon \lambda}+2 M_{v}^{2} \varepsilon \lambda+\frac{\mu}{2}\left|x_{\varepsilon, \mu}\right|_{X}^{2},
$$

for all constant $\lambda>0$. From the inequality

$$
\Phi_{\varepsilon, \mu}\left(t_{\varepsilon, \mu}, s_{\varepsilon, \mu}, x_{\varepsilon, \mu}, x_{\varepsilon, \mu}\right) \leq \Phi_{\varepsilon, \mu}\left(t_{\varepsilon, \mu}, s_{\varepsilon, \mu}, x_{\varepsilon, \mu}, y_{\varepsilon, \mu}\right)
$$

we deduce

$$
\frac{1}{2 \varepsilon}\left((-A)^{-1}\left(x_{\varepsilon, \mu}-y_{\varepsilon, \mu}\right) \mid\left(x_{\varepsilon, \mu}-y_{\varepsilon, \mu}\right)\right)_{X} \leq v_{\sigma}\left(s_{\varepsilon, \mu}, x_{\varepsilon, \mu}\right)-v_{\sigma}\left(s_{\varepsilon, \mu}, y_{\varepsilon, \mu}\right)+\frac{\mu}{2}\left|x_{\varepsilon, \mu}\right|_{X}^{2} .
$$


With (ii) in Definition 3.2 we have:

$$
\frac{1}{\varepsilon}\left|(-A)^{-\frac{1}{2}}\left(x_{\varepsilon, \mu}-y_{\varepsilon, \mu}\right)\right|_{X}^{2} \leq 2 M_{v}\left|x_{\varepsilon, \mu}-y_{\varepsilon, \mu}\right|+\mu\left|x_{\varepsilon, \mu}\right|_{X}^{2}
$$

Estimate (3.14) follows from Young's inequality.

Step 4. We are going to show that $\min \left(t_{\varepsilon, \mu}, s_{\varepsilon, \mu}\right)=\tau$. If it is not true, then $t_{\varepsilon, \mu}>\tau$ and $s_{\varepsilon, \mu}>\tau$. Let $\varphi$ and $\psi$ be two mappings defined by:

$$
\begin{aligned}
& \varphi(t, x)=v_{\sigma}\left(s_{\varepsilon, \mu}, y_{\varepsilon, \mu}\right)+\frac{1}{2 \varepsilon}\left((-A)^{-1}\left(x-y_{\varepsilon, \mu}\right) \mid\left(x-y_{\varepsilon, \mu}\right)\right)_{X}+\frac{\left(t-s_{\varepsilon, \mu}\right)^{2}}{2 \varepsilon^{\frac{2}{\bar{\eta}}}}+\frac{\mu}{2}\left(|x|_{X}^{2}+\left|y_{\varepsilon, \mu}\right|_{X}^{2}\right) \\
& \psi(t, y)=w_{\sigma}\left(t_{\varepsilon, \mu}, x_{\varepsilon, \mu}\right)-\frac{1}{2 \varepsilon}\left((-A)^{-1}\left(x_{\varepsilon, \mu}-y\right) \mid\left(x_{\varepsilon, \mu}-y\right)\right)_{X}-\frac{\left(t_{\varepsilon, \mu}-s\right)^{2}}{2 \varepsilon^{\frac{2}{\bar{\eta}}}}-\frac{\mu}{2}\left(\left|x_{\varepsilon, \mu}\right|_{X}^{2}+|y|_{X}^{2}\right) .
\end{aligned}
$$

The mappings $\varphi, \psi$ belong to $C_{A}^{1}(] 0, T[\times X)$. Indeed

(i) $\varphi \in C^{1}(] 0, T[\times X)$ and $D_{x} \varphi(t, x)=\frac{1}{\varepsilon}(-A)^{-1}\left(x-y_{\varepsilon, \mu}\right)+\mu x$.

(ii) $D_{x} \varphi(\cdot, x)$ is constant in $t$, and $x \mapsto D_{x} \varphi(t, x)$ is Lipschitz from $X$ into $X$ because $(-A)^{-1}$ is a linear and continuous operator from $X$ into $X$ :

$$
\left|D_{x} \varphi(t, x)-D_{x} \varphi(t, y)\right|_{X}^{2} \leq 2\left(\frac{1}{\varepsilon}\left|A^{-1}(x-y)\right|_{X}^{2}+\mu|x-y|_{X}^{2}\right) \leq 2\left(\mu+\frac{C}{\varepsilon}\right)|x-y|_{X}^{2} .
$$

(iii) It is clear that for all $\theta \in\left[0,1-\alpha\left[, D_{x} \varphi(t, x) \in D\left((-A)^{\theta}\right) \Leftrightarrow x \in D\left((-A)^{\theta}\right)\right.\right.$.

(iv) Moreover the mapping $x \mapsto D_{x} \varphi(t, x)$ is continuous from $D\left((-A)^{\frac{1}{2}}\right)$ into itself.

The mappings $\varphi, \psi$ have been chosen to satisfy:

$$
\left(t_{\varepsilon, \mu}, x_{\varepsilon, \mu}\right) \in \arg \max _{Q_{\tau}}\left(w_{\sigma}-\varphi\right), \quad \text { and } \quad\left(s_{\varepsilon, \mu}, y_{\varepsilon, \mu}\right) \in \arg \min _{Q_{\tau}}\left(v_{\sigma}-\psi\right)
$$

Since $v_{\sigma}$ and $w_{\sigma}$ satisfy (iii) in Definition 3.2, with [5, Cor. 3.4], $D_{x}^{+} w_{\sigma}\left(t_{\varepsilon, \mu}, x_{\varepsilon, \mu}\right)$ and $D_{x}^{-} v_{\sigma}\left(s_{\varepsilon, \mu}, y_{\varepsilon, \mu}\right)$ are included in $D\left((-A)^{\theta}\right)$ for all $\theta \in\left[0, \frac{3}{4}\left[\right.\right.$. Due to Lemma 3.6, $D_{x} \varphi\left(t_{\varepsilon, \mu}, x_{\varepsilon, \mu}\right) \subset D_{x}^{+} w_{\sigma}\left(t_{\varepsilon, \mu}, x_{\varepsilon, \mu}\right)$ and $D_{x} \psi\left(s_{\varepsilon, \mu}, y_{\varepsilon, \mu}\right) \subset D_{x}^{-} v_{\sigma}\left(s_{\varepsilon, \mu}, y_{\varepsilon, \mu}\right)$. Hence, we have

$$
D_{x} \varphi\left(t_{\varepsilon, \mu}, x_{\varepsilon, \mu}\right), D_{x} \psi\left(s_{\varepsilon, \mu}, y_{\varepsilon, \mu}\right) \in D\left((-A)^{\theta}\right) \text { for all } \theta \in[0,1-\alpha[\text {. }
$$

Therefore $x_{\varepsilon, \mu}$ and $y_{\varepsilon, \mu}$ belong to $D\left((-A)^{\theta}\right)$ for all $\theta \in\left[0,1-\alpha\left[\right.\right.$, and in particular $x_{\varepsilon, \mu}, y_{\varepsilon, \mu} \in D\left((-A)^{\frac{1}{2}}\right)$. Since $w_{\sigma}$ is a viscosity subsolution of $(3.7)$, and $\left(t_{\varepsilon, \mu}, x_{\varepsilon, \mu}\right)$ belongs to (] $0, T\left[\times D\left((-A)^{\frac{1}{2}}\right)\right) \cap \arg \max \left(w_{\sigma}-\varphi\right)$, we have:

$$
\begin{aligned}
\frac{t_{\varepsilon, \mu}-s_{\varepsilon, \mu}}{\varepsilon^{\frac{2}{\bar{\eta}}}}+H\left(t_{\varepsilon, \mu},\right. & \left.x_{\varepsilon, \mu},(-A)^{\beta}\left(\frac{1}{\varepsilon}(-A)^{-1}\left(x_{\varepsilon, \mu}-y_{\varepsilon, \mu}\right)+\mu x_{\varepsilon, \mu}\right)\right) \\
& +\left((-A)^{\frac{1}{2}}\left(\frac{1}{\varepsilon}(-A)^{-1}\left(x_{\varepsilon, \mu}-y_{\varepsilon, \mu}\right)+\mu x_{\varepsilon, \mu}\right) \mid(-A)^{\frac{1}{2}} x_{\varepsilon, \mu}\right)_{X} \\
& +\left((-A)^{\beta}\left(\frac{1}{\varepsilon}(-A)^{-1}\left(x_{\varepsilon, \mu}-y_{\varepsilon, \mu}\right)+\mu x_{\varepsilon, \mu}\right) \mid F\left(t_{\varepsilon, \mu}, \Lambda x_{\varepsilon, \mu}\right)\right)_{X} \\
\leq & -\frac{\sigma}{T^{2}} .
\end{aligned}
$$


In the same way, since $v_{\sigma}$ is a viscosity supersolution of (3.8), with (3.15) we have:

$$
\begin{aligned}
\frac{t_{\varepsilon, \mu}-s_{\varepsilon, \mu}}{\varepsilon^{\frac{2}{\eta}}}+H\left(s_{\varepsilon, \mu}, y_{\varepsilon, \mu},(-A)^{\beta}\left(\frac{1}{\varepsilon}(-A)^{-1}\left(x_{\varepsilon, \mu}-y_{\varepsilon, \mu}\right)-\mu y_{\varepsilon, \mu}\right)\right) \\
+\left((-A)^{\frac{1}{2}}\left(\frac{1}{\varepsilon}(-A)^{-1}\left(x_{\varepsilon, \mu}-y_{\varepsilon, \mu}\right)-\mu y_{\varepsilon, \mu}\right) \mid(-A)^{\frac{1}{2}} y_{\varepsilon, \mu}\right)_{X} \\
\quad+\left((-A)^{\beta}\left(\frac{1}{\varepsilon}(-A)^{-1}\left(x_{\varepsilon, \mu}-y_{\varepsilon, \mu}\right)+\mu y_{\varepsilon, \mu}\right) \mid F\left(s_{\varepsilon, \mu}, \Lambda y_{\varepsilon, \mu}\right)\right)_{X} \\
\geq \frac{\sigma}{T^{2}} .
\end{aligned}
$$

Substracting the previous two inequalities we obtain

$$
\begin{aligned}
H\left(t_{\varepsilon, \mu}, x_{\varepsilon, \mu},(-A)^{\beta}(\right. & \left.\left.\frac{1}{\varepsilon}(-A)^{-1}\left(x_{\varepsilon, \mu}-y_{\varepsilon, \mu}\right)+\mu x_{\varepsilon, \mu}\right)\right) \\
& -H\left(s_{\varepsilon, \mu}, y_{\varepsilon, \mu},(-A)^{\beta}\left(\frac{1}{\varepsilon}(-A)^{-1}\left(x_{\varepsilon, \mu}-y_{\varepsilon, \mu}\right)-\mu y_{\varepsilon, \mu}\right)\right) \\
& +\left((-A)^{\frac{1}{2}}\left(\frac{1}{\varepsilon}(-A)^{-1}\left(x_{\varepsilon, \mu}-y_{\varepsilon, \mu}\right)\right) \mid(-A)^{\frac{1}{2}}\left(x_{\varepsilon, \mu}-y_{\varepsilon, \mu}\right)\right)_{X} \\
& +\mu\left|(-A)^{\frac{1}{2}} x_{\varepsilon, \mu}\right|_{X}^{2}+\mu\left|(-A)^{\frac{1}{2}} y_{\varepsilon, \mu}\right|_{X}^{2} \\
& +\left((-A)^{\beta}\left(\frac{1}{\varepsilon}(-A)^{-1}\left(x_{\varepsilon, \mu}-y_{\varepsilon, \mu}\right)\right) \mid F\left(t_{\varepsilon, \mu}, \Lambda x_{\varepsilon, \mu}\right)-F\left(s_{\varepsilon, \mu}, \Lambda y_{\varepsilon, \mu}\right)\right)_{X} \\
& +\mu\left((-A)^{\beta} x_{\varepsilon, \mu} \mid F\left(t_{\varepsilon, \mu}, \Lambda x_{\varepsilon, \mu}\right)\right)_{X}-\mu\left((-A)^{\beta} y_{\varepsilon, \mu} \mid F\left(s_{\varepsilon, \mu}, \Lambda y_{\varepsilon, \mu}\right)\right)_{X} \\
\leq & -\frac{2 \sigma}{T^{2}} .
\end{aligned}
$$

Thus we have

$$
\begin{aligned}
\mu\left|(-A)^{\frac{1}{2}} x_{\varepsilon, \mu}\right|_{X}^{2}+\mu \mid & \left.(-A)^{\frac{1}{2}} y_{\varepsilon, \mu}\right|_{X} ^{2}+\frac{1}{\varepsilon}\left|x_{\varepsilon, \mu}-y_{\varepsilon, \mu}\right|_{X}^{2} \\
\leq & -\frac{2 \sigma}{T^{2}}+K_{H}\left(\left|t_{\varepsilon, \mu}-s_{\varepsilon, \mu}\right|^{\eta_{2}}+\left|x_{\varepsilon, \mu}-y_{\varepsilon, \mu}\right|_{X}+\mu\left|(-A)^{\beta}\left(x_{\varepsilon, \mu}+y_{\varepsilon, \mu}\right)\right|_{X}\right) \\
& +\frac{1}{\varepsilon}\left|(-A)^{\beta-1}\left(x_{\varepsilon, \mu}-y_{\varepsilon, \mu}\right)\right|_{X}\left|F\left(t_{\varepsilon, \mu}, \Lambda x_{\varepsilon, \mu}\right)-F\left(s_{\varepsilon, \mu}, \Lambda y_{\varepsilon, \mu}\right)\right|_{X} \\
& +\mu\left|(-A)^{\beta} x_{\varepsilon, \mu}\right|_{X}\left|F\left(t_{\varepsilon, \mu}, \Lambda x_{\varepsilon, \mu}\right)\right|_{X}+\mu\left|(-A)^{\beta} y_{\varepsilon, \mu}\right|_{X}\left|F\left(s_{\varepsilon, \mu}, \Lambda y_{\varepsilon, \mu}\right)\right|_{X} .
\end{aligned}
$$

\section{Estimates of (3.17)-(3.19):}

Estimate of (3.17). With (3.11) and Young's inequality we can write

$$
\left|t_{\varepsilon, \mu}-s_{\varepsilon, \mu}\right|^{\eta_{2}} \leq C \varepsilon^{\frac{\eta_{2}}{\eta}} \leq C \varepsilon \quad \text { and } \quad K_{H}\left|x_{\varepsilon, \mu}-y_{\varepsilon, \mu}\right|_{X} \leq 2 \varepsilon K_{H}^{2}+\frac{\left|x_{\varepsilon, \mu}-y_{\varepsilon, \mu}\right|_{X}^{2}}{8 \varepsilon}
$$


For all $\sigma>0$, with (2.13), we have:

$$
\begin{aligned}
\mu K_{H}\left|(-A)^{\beta}\left(x_{\varepsilon, \mu}+y_{\varepsilon, \mu}\right)\right|_{X} & \leq \mu K_{H}\left(\left|(-A)^{\beta} x_{\varepsilon, \mu}\right|_{X}+\left|(-A)^{\beta} y_{\varepsilon, \mu}\right|_{X}\right) \\
& \leq \mu K_{H}\left[\sigma\left(\left|(-A)^{\frac{1}{2}} x_{\varepsilon, \mu}\right|_{X}+\left|(-A)^{\frac{1}{2}} y_{\varepsilon, \mu}\right|_{X}\right)+C_{\beta, \sigma}\left(\left|x_{\varepsilon, \mu}\right|_{X}+\left|y_{\varepsilon, \mu}\right|_{X}\right)\right] \\
& \leq \mu K_{H}\left[\frac{1}{2} \sigma\left(\left|(-A)^{\frac{1}{2}} x_{\varepsilon, \mu}\right|_{X}^{2}+\left|(-A)^{\frac{1}{2}} y_{\varepsilon, \mu}\right|_{X}^{2}+2\right)+C_{\beta, \sigma}\left(\left|x_{\varepsilon, \mu}\right|_{X}+\left|y_{\varepsilon, \mu}\right|_{X}\right)\right] .
\end{aligned}
$$

Choosing $\sigma=\frac{1}{K_{H}}$ we have:

$$
\mu K_{H}\left|(-A)^{\beta}\left(x_{\varepsilon, \mu}+y_{\varepsilon, \mu}\right)\right|_{X} \leq \mu\left[\frac{1}{2}\left|(-A)^{\frac{1}{2}} x_{\varepsilon, \mu}\right|_{X}^{2}+\frac{1}{2}\left|(-A)^{\frac{1}{2}} y_{\varepsilon, \mu}\right|_{X}^{2}+1+K_{H} C_{\beta, 1 / K_{H}}\left(\left|x_{\varepsilon, \mu}\right|_{X}+\left|y_{\varepsilon, \mu}\right|_{X}\right)\right]
$$

With (3.20) and (3.21), we have:

$$
\begin{aligned}
(3.17) \leq & K_{H} C \varepsilon+2 \varepsilon K_{H}^{2}+\frac{\left|x_{\varepsilon, \mu}-y_{\varepsilon, \mu}\right|_{X}^{2}}{8 \varepsilon} \\
& +\mu\left[\frac{1}{2}\left|(-A)^{\frac{1}{2}} x_{\varepsilon, \mu}\right|_{X}^{2}+\frac{1}{2}\left|(-A)^{\frac{1}{2}} y_{\varepsilon, \mu}\right|_{X}^{2}+1+K_{H} C_{\beta, 1 / K_{H}}\left(\left|x_{\varepsilon, \mu}\right|_{X}+\left|y_{\varepsilon, \mu}\right|_{X}\right)\right] .
\end{aligned}
$$

Estimate of (3.18). We first write

$$
(3.18) \leq \frac{1}{\varepsilon}\left\|(-A)^{\beta-\frac{1}{2}}\right\|\left|(-A)^{-\frac{1}{2}}\left(x_{\varepsilon, \mu}-y_{\varepsilon, \mu}\right)\right|_{X}\left|F\left(t_{\varepsilon, \mu}, \Lambda x_{\varepsilon, \mu}\right)-F\left(s_{\varepsilon, \mu}, \Lambda y_{\varepsilon, \mu}\right)\right|_{X} .
$$

From (2.2) and (2.4), it yields

$$
\begin{aligned}
\left|F\left(t_{\varepsilon, \mu}, \Lambda x_{\varepsilon, \mu}\right)-F\left(s_{\varepsilon, \mu}, \Lambda y_{\varepsilon, \mu}\right)\right|_{X} \leq & M_{1, F}\left(1+\left|\Lambda x_{\varepsilon, \mu}\right| X_{0}\right)\left|t_{\varepsilon, \mu}-s_{\varepsilon, \mu}\right|^{\eta_{1}}+K_{F}\left|\Lambda x_{\varepsilon, \mu}-\Lambda y_{\varepsilon, \mu}\right|_{X_{0}} \\
\leq & M_{1, F}\left(1+C_{1 / 2}\left|(-A)^{\frac{1}{2}} x_{\varepsilon, \mu}\right|_{X}\right)\left|t_{\varepsilon, \mu}-s_{\varepsilon, \mu}\right|^{\eta_{1}} \\
& +K_{F} C_{\alpha}\left|(-A)^{\alpha}\left(x_{\varepsilon, \mu}-y_{\varepsilon, \mu}\right)\right|_{X} .
\end{aligned}
$$

Then, choosing $\alpha_{0}>0$ such that $\alpha+\alpha_{0}<\frac{1}{2}$, we obtain

$$
\begin{aligned}
(3.18) \leq & C \frac{\left|(-A)^{-\frac{1}{2}}\left(x_{\varepsilon, \mu}-y_{\varepsilon, \mu}\right)\right|_{X}}{\varepsilon^{1-\alpha_{0}}} \frac{\left|(-A)^{\alpha}\left(x_{\varepsilon, \mu}-y_{\varepsilon, \mu}\right)\right|_{X}}{\varepsilon^{\alpha_{0}}} \\
& +C \frac{\left|(-A)^{-\frac{1}{2}}\left(x_{\varepsilon, \mu}-y_{\varepsilon, \mu}\right)\right|_{X}}{\varepsilon^{1-\alpha_{0}}}\left(1+\left|(-A)^{\frac{1}{2}} x_{\varepsilon, \mu}\right|_{X}\right) \frac{\left|t_{\varepsilon, \mu}-s_{\varepsilon, \mu}\right|^{\eta_{1}}}{\varepsilon^{\alpha_{0}}} .
\end{aligned}
$$

Estimate of (3.23). We first estimate the factor $\left|(-A)^{-\frac{1}{2}}\left(x_{\varepsilon, \mu}-y_{\varepsilon, \mu}\right)\right|_{X} / \varepsilon^{1-\alpha_{0}}$. From inequality (3.10) it follows that

$$
\frac{1}{\varepsilon}\left|(-A)^{-\frac{1}{2}}\left(x_{\varepsilon, \mu}-y_{\varepsilon, \mu}\right)\right|_{X}^{2} \leq w\left(t_{\varepsilon, \mu}, x_{\varepsilon, \mu}\right)-w\left(s_{\varepsilon, \mu}, y_{\varepsilon, \mu}\right)+v\left(t_{\varepsilon, \mu}, x_{\varepsilon, \mu}\right)-v\left(s_{\varepsilon, \mu}, y_{\varepsilon, \mu}\right) .
$$

As $t_{\varepsilon, \mu}, s_{\varepsilon, \mu} \in[\tau, T-\tau]$ and $[\tau, T-\tau]$ is compact, with properties (iii) and (iv) in Definition 3.2, setting $C(v, w)=M_{1, v}+M_{1, w}$ and $C(\tau)=\sup _{t \in[\tau, T]} C_{t, 1 / 2, v}+C_{t, 1 / 2, w}$, we have

$$
\frac{1}{\varepsilon}\left|(-A)^{-\frac{1}{2}}\left(x_{\varepsilon, \mu}-y_{\varepsilon, \mu}\right)\right|_{X}^{2} \leq 2 C(v, w)\left(1+\left|(-A)^{\frac{1}{2}} x_{\varepsilon, \mu}\right|_{X}\right)\left|t_{\varepsilon, \mu}-s_{\varepsilon, \mu}\right|^{\eta}+C(\tau)\left|(-A)^{-\frac{1}{2}}\left(x_{\varepsilon, \mu}-y_{\varepsilon, \mu}\right)\right|_{X}
$$


As

$$
C(\tau)\left|(-A)^{-\frac{1}{2}}\left(x_{\varepsilon, \mu}-y_{\varepsilon, \mu}\right)\right|_{X} \leq \frac{\left|(-A)^{-\frac{1}{2}}\left(x_{\varepsilon, \mu}-y_{\varepsilon, \mu}\right)\right|_{X}^{2}}{2 \varepsilon}+\frac{\varepsilon}{2} C(\tau)^{2}
$$

then

$$
\frac{1}{\varepsilon}\left|(-A)^{-\frac{1}{2}}\left(x_{\varepsilon, \mu}-y_{\varepsilon, \mu}\right)\right|_{X}^{2} \leq 4 C(v, w)\left(1+\left|(-A)^{\frac{1}{2}} x_{\varepsilon, \mu}\right|_{X}\right)\left|t_{\varepsilon, \mu}-s_{\varepsilon, \mu}\right|^{\eta}+\varepsilon C(\tau)^{2} .
$$

Hence, we obtain:

$$
\frac{1}{\varepsilon^{1-\alpha_{0}}}\left|(-A)^{-\frac{1}{2}}\left(x_{\varepsilon, \mu}-y_{\varepsilon, \mu}\right)\right|_{X} \leq 2 \sqrt{C(v, w)\left(1+\left|(-A)^{\frac{1}{2}} x_{\varepsilon, \mu}\right|_{X}\right)} \frac{\left|t_{\varepsilon, \mu}-s_{\varepsilon, \mu}\right|^{\frac{\eta}{2}}}{\varepsilon^{\frac{1}{2}-\alpha_{0}}}+\varepsilon^{\alpha_{0}} C(\tau) .
$$

We now estimate the factor $\left|(-A)^{\alpha}\left(x_{\varepsilon, \mu}-y_{\varepsilon, \mu}\right)\right|_{X} / \varepsilon^{\alpha_{0}}$. With (2.12), we have:

$$
\frac{1}{\varepsilon^{\alpha_{0}}}\left|(-A)^{\alpha}\left(x_{\varepsilon, \mu}-y_{\varepsilon, \mu}\right)\right|_{X} \leq M_{\alpha, 1 / 2}\left|(-A)^{\frac{1}{2}}\left(x_{\varepsilon, \mu}-y_{\varepsilon, \mu}\right)\right|_{X}^{2 \alpha} \frac{\left|x_{\varepsilon, \mu}-y_{\varepsilon, \mu}\right|_{X}^{1-2 \alpha}}{\varepsilon^{\frac{1}{2}-\alpha_{0}}} .
$$

Applying Young's inequality (3.5) to the left hand side and taking $q=1 /(1-2 \alpha), p=1 / 2 \alpha, q \lambda^{q}=\varepsilon^{\frac{1}{2}-\frac{\alpha_{0}}{1-2 \alpha}}$, then $\frac{\lambda^{p}}{p}=C \varepsilon^{\left(1-2 \alpha-2 \alpha_{0}\right) / 4 \alpha}$ and we obtain:

$$
\frac{1}{\varepsilon^{\alpha_{0}}}\left|(-A)^{\alpha}\left(x_{\varepsilon, \mu}-y_{\varepsilon, \mu}\right)\right|_{X} \leq \frac{\left|x_{\varepsilon, \mu}-y_{\varepsilon, \mu}\right|_{X}}{\varepsilon^{1 / 2}}+C \varepsilon^{\left(1-2 \alpha-2 \alpha_{0}\right) / 4 \alpha}\left|(-A)^{\frac{1}{2}}\left(x_{\varepsilon, \mu}-y_{\varepsilon, \mu}\right)\right|_{X} .
$$

The exponent of $\left(1-2 \alpha-2 \alpha_{0}\right) / 4 \alpha$ of $\varepsilon$ is positive because $\alpha+\alpha_{0}<\frac{1}{2}$.

With (3.25), (3.26), and with Young's inequality we can write

$(3.23) \leq\left[C\left(1+\left|(-A)^{\frac{1}{2}} x_{\varepsilon, \mu}\right|_{X}\right) \frac{\left|t_{\varepsilon, \mu}-s_{\varepsilon, \mu}\right|^{\eta}}{\varepsilon^{1-2 \alpha_{0}}}+\varepsilon^{2 \alpha_{0}} C(\tau)\right]$

$$
+\frac{\left|x_{\varepsilon, \mu}-y_{\varepsilon, \mu}\right|_{X}^{2}}{8 \varepsilon}+C \varepsilon^{\frac{\left(1-2 \alpha-2 \alpha_{0}\right)}{2 \alpha}}\left|(-A)^{\frac{1}{2}}\left(x_{\varepsilon, \mu}-y_{\varepsilon, \mu}\right)\right|_{X}^{2} .
$$

With (3.11) one has

$$
\frac{\left|t_{\varepsilon, \mu}-s_{\varepsilon, \mu}\right|^{\eta}}{\varepsilon^{1-2 \alpha_{0}}} \leq C \frac{\varepsilon^{\frac{\eta}{\eta}}}{\varepsilon^{1-2 \alpha_{0}}} \leq C \varepsilon^{2 \alpha_{0}}
$$

which gives

$$
(3.23) \leq C\left(\varepsilon^{2 \alpha_{0}}+\varepsilon^{\frac{\left(1-2 \alpha-2 \alpha_{0}\right)}{2 \alpha}}\right)\left(1+\left|(-A)^{\frac{1}{2}} x_{\varepsilon, \mu}\right|_{X}^{2}+\left|(-A)^{\frac{1}{2}} y_{\varepsilon, \mu}\right|_{X}^{2}\right)+\varepsilon^{2 \alpha_{0}} C(\tau)+\frac{\left|x_{\varepsilon, \mu}-y_{\varepsilon, \mu}\right|_{X}^{2}}{8 \varepsilon} .
$$

Estimate of (3.24). We finally estimate (3.24) with (3.25), (3.26) and (3.11), and we have

$$
\begin{aligned}
(3.24) & \leq C(\tau)\left(1+\left|(-A)^{\frac{1}{2}} x_{\varepsilon, \mu}\right|_{X}\right)^{3 / 2}\left(\frac{\left|t_{\varepsilon, \mu}-s_{\varepsilon, \mu}\right|^{\frac{\eta}{2}+\eta_{1}}}{\varepsilon^{\frac{1}{2}}}+\left|t_{\varepsilon, \mu}-s_{\varepsilon, \mu}\right|^{\eta_{1}}\right) \\
& \leq C(\tau) \varepsilon\left(1+\left|(-A)^{\frac{1}{2}} x_{\varepsilon, \mu}\right|_{X}^{2}\right) .
\end{aligned}
$$


We complete the estimate of (3.18) with (3.27) and (3.28):

$$
\begin{aligned}
(3.18) \leq & C\left(\varepsilon+\varepsilon^{2 \alpha_{0}}+\varepsilon^{\left(1-2 \alpha-2 \alpha_{0}\right) / 2 \alpha}\right)\left(1+\left|(-A)^{\frac{1}{2}} x_{\varepsilon, \mu}\right|_{X}^{2}+\left|(-A)^{\frac{1}{2}} y_{\varepsilon, \mu}\right|_{X}^{2}\right) \\
& +C(\tau) \varepsilon^{2 \alpha_{0}}+\frac{\left|x_{\varepsilon, \mu}-y_{\varepsilon, \mu}\right|_{X}^{2}}{8 \varepsilon} .
\end{aligned}
$$

Estimate of ( 3.19). Since $F$ is bounded, as in (3.17), and with (3.21) we obtain

$$
(3.19) \leq \mu\left[\frac{1}{8}\left|(-A)^{\frac{1}{2}} x_{\varepsilon, \mu}\right|_{X}^{2}+\frac{1}{2}+\frac{1}{8}\left|(-A)^{\frac{1}{2}} y_{\varepsilon, \mu}\right|_{X}^{2}+\left(\left|x_{\varepsilon, \mu}\right|_{X}^{2}+\left|y_{\varepsilon, \mu}\right|_{X}^{2}\right)+C\right]
$$

\section{End of step 4}

Collecting the different estimates of the terms in the right hand side of (3.16) we obtain:

$$
\begin{array}{r}
\left(\mu-\frac{\mu}{2}-C\left(\varepsilon^{2 \alpha_{0}}+\varepsilon^{\left(1-2 \alpha-2 \alpha_{0}\right) / 2 \alpha}\right)-\frac{\mu}{8}\right)\left[\left|(-A)^{\frac{1}{2}} x_{\varepsilon, \mu}\right|_{X}^{2}+\left|(-A)^{\frac{1}{2}} y_{\varepsilon, \mu}\right|_{X}^{2}\right]^{2}+\frac{1}{\varepsilon}\left(1-\frac{1}{8}-\frac{1}{4}-\frac{1}{8}\right)\left|x_{\varepsilon, \mu}-y_{\varepsilon, \mu}\right|_{X}^{2} \\
\leq-\frac{2 \sigma}{T^{2}}+\mu C\left(1+\left|x_{\varepsilon, \mu}\right|_{X}^{2}+\left|y_{\varepsilon, \mu}\right|_{X}^{2}\right)+C(\tau)\left[\varepsilon^{\frac{1}{\bar{\eta}}}+\varepsilon^{2 \alpha_{0}}\right]
\end{array}
$$

We take $\varepsilon(\mu)$ small enough to have $C\left(\varepsilon^{2 \alpha_{0}}+\varepsilon^{\left(1-2 \alpha-2 \alpha_{0}\right) / 2 \alpha}\right)<\frac{3 \mu}{8}$, and we take the limit when $\mu$ tends to zero. We obtain the contradiction $0 \leq-\frac{2 \sigma}{T^{2}}$. Thus the equality $\min \left(t_{\varepsilon, \mu}, s_{\varepsilon, \mu}\right)=\tau$ is established.

Step 5. We are going to conclude with the initial data. We argue by contradiction. If (3.6) does not hold, then there exists $\left.\left(t_{0}, x_{0}\right) \in\right] 0, T[\times X$ such that

$$
0<w_{\sigma}\left(t_{0}, x_{0}\right)-v_{\sigma}\left(t_{0}, x_{0}\right)=\delta_{0} .
$$

We choose $\tau$ and $\varepsilon$ small enough to have $\left(t_{0}, x_{0}\right) \in Q_{\tau}$, and

$$
\left[w(t, x)-g\left(\mathrm{e}^{t A} x\right)\right]^{+} \leq \frac{\delta_{0}}{8} \quad \text { and } \quad\left[v(t, x)-g\left(\mathrm{e}^{t A} x\right)\right]^{-} \leq \frac{\delta_{0}}{8}
$$

for all $x \in X$ and all $t \in\left[0, \tau+C \varepsilon^{1 / \bar{\eta}}\right]$, where $C$ is the constant in (3.11). One has

$$
\Phi_{\varepsilon, \mu}\left(t_{0}, t_{0}, x_{0}, x_{0}\right) \leq \Phi_{\varepsilon, \mu}\left(t_{\varepsilon, \mu}, s_{\varepsilon, \mu}, x_{\varepsilon, \mu}, y_{\varepsilon, \mu}\right) \leq w_{\sigma}\left(t_{\varepsilon, \mu}, x_{\varepsilon, \mu}\right)-v_{\sigma}\left(s_{\varepsilon, \mu}, y_{\varepsilon, \mu}\right),
$$

i.e.

$$
\begin{aligned}
w_{\sigma}\left(t_{0}, x_{0}\right)-v_{\sigma}\left(t_{0}, x_{0}\right)-\mu\left|x_{0}\right|_{X}^{2} \leq & w_{\sigma}\left(t_{\varepsilon, \mu}, x_{\varepsilon, \mu}\right)-v_{\sigma}\left(s_{\varepsilon, \mu}, y_{\varepsilon, \mu}\right) \\
= & {\left[w_{\sigma}\left(t_{\varepsilon, \mu}, x_{\varepsilon, \mu}\right)+\frac{\sigma}{T-t_{\varepsilon, \mu}}-g\left(\mathrm{e}^{t_{\varepsilon, \mu} A} x_{\varepsilon, \mu}\right)\right]+g\left(\mathrm{e}^{t_{\varepsilon, \mu} A} x_{\varepsilon, \mu}\right)-g\left(\mathrm{e}^{s_{\varepsilon, \mu} A} y_{\varepsilon, \mu}\right) } \\
& +\left[g\left(\mathrm{e}^{s_{\varepsilon, \mu} A} y_{\varepsilon, \mu}\right)+\frac{\sigma}{T-s_{\varepsilon, \mu}}-v_{\sigma}\left(s_{\varepsilon, \mu}, y_{\varepsilon, \mu}\right)\right]-\left[\frac{\sigma}{T-s_{\varepsilon, \mu}}+\frac{\sigma}{T-t_{\varepsilon, \mu}}\right] \\
\leq & {\left[w\left(t_{\varepsilon, \mu}, x_{\varepsilon, \mu}\right)-g\left(\mathrm{e}^{t_{\varepsilon, \mu} A} x_{\varepsilon, \mu}\right)\right]^{+}+K_{g}\left|\mathrm{e}^{t_{\varepsilon, \mu} A} x_{\varepsilon, \mu}-\mathrm{e}^{s_{\varepsilon, \mu} A} y_{\varepsilon, \mu}\right| } \\
& +\left[v\left(s_{\varepsilon, \mu}, y_{\varepsilon, \mu}\right)-g\left(\mathrm{e}^{s_{\varepsilon, \mu} A} y_{\varepsilon, \mu}\right)\right]^{-} .
\end{aligned}
$$

Since $0<\tau=\min \left(t_{\varepsilon, \mu}, s_{\varepsilon, \mu}\right)$ and $\left|t_{\varepsilon, \mu}-s_{\varepsilon, \mu}\right| \leq C \varepsilon^{1 / \bar{\eta}}$, we have

$$
\left[w\left(t_{\varepsilon, \mu}, x_{\varepsilon, \mu}\right)-g\left(\mathrm{e}^{t_{\varepsilon, \mu} A} x_{\varepsilon, \mu}\right)\right]^{+} \leq \frac{\delta_{0}}{8} \quad \text { and } \quad\left[v\left(s_{\varepsilon, \mu}, y_{\varepsilon, \mu}\right)-g\left(\mathrm{e}^{s_{\varepsilon, \mu} A} y_{\varepsilon, \mu}\right)\right]^{-} \leq \frac{\delta_{0}}{8}
$$


Still using $\tau=\min \left(t_{\varepsilon, \mu}, s_{\varepsilon, \mu}\right)$, with (2.11), we can write

$$
\begin{aligned}
\left|\mathrm{e}^{t_{\varepsilon, \mu} A} x_{\varepsilon, \mu}-\mathrm{e}^{s_{\varepsilon, \mu} A} y_{\varepsilon, \mu}\right|_{X} & \leq\left|\left(\mathrm{e}^{t_{\varepsilon, \mu} A}-\mathrm{e}^{s_{\varepsilon, \mu} A}\right) x_{\varepsilon, \mu}\right|_{X}+\left|\mathrm{e}^{s_{\varepsilon, \mu} A}\left(x_{\varepsilon, \mu}-y_{\varepsilon, \mu}\right)\right|_{X} \\
& \leq\left|\left(\mathrm{e}^{\left|t_{\varepsilon, \mu}-s_{\varepsilon, \mu}\right| A}-I\right) \mathrm{e}^{\tau A} x_{\varepsilon, \mu}\right|_{X}+\left|(-A)^{\frac{1}{2}} \mathrm{e}^{s_{\varepsilon, \mu} A}(-A)^{-\frac{1}{2}}\left(x_{\varepsilon, \mu}-y_{\varepsilon, \mu}\right)\right|_{X} \\
& \leq M_{0}\left|t_{\varepsilon, \mu}-s_{\varepsilon, \mu}\right|\left|A \mathrm{e}^{\tau A} x_{\varepsilon, \mu}\right|_{X}+\frac{C}{\sqrt{\tau}}\left|(-A)^{-\frac{1}{2}}\left(x_{\varepsilon, \mu}-y_{\varepsilon, \mu}\right)\right|_{X} \\
& \leq \frac{C}{\tau}\left|x_{\varepsilon, \mu}\right|_{X}\left|t_{\varepsilon, \mu}-s_{\varepsilon, \mu}\right|+\frac{C}{\sqrt{\tau}}\left|(-A)^{-\frac{1}{2}}\left(x_{\varepsilon, \mu}-y_{\varepsilon, \mu}\right)\right|_{X} \\
& \leq \frac{C}{\tau}\left|x_{\varepsilon, \mu}\right|_{X} \varepsilon^{\frac{1}{\eta}}+\frac{C}{\sqrt{\tau}} \sqrt{\varepsilon} \leq \frac{C}{\tau} \frac{\varepsilon^{\frac{1}{\eta}}}{\mu^{1 / 2}}+\frac{C}{\sqrt{\tau}} \sqrt{\varepsilon} .
\end{aligned}
$$

(The last inequality is obtained with (3.12).) We choose $\mu \leq \frac{\delta_{0}}{4 K_{g}}$, and next $\varepsilon$ such that

$$
\frac{C}{\tau} \frac{\varepsilon^{\frac{1}{\eta}}}{\mu^{1 / 2}}+\frac{C}{\sqrt{\tau}} \sqrt{\varepsilon} \leq \mu
$$

We obtain:

$$
\left|\mathrm{e}^{t_{\varepsilon, \mu} A} x_{\varepsilon, \mu}-\mathrm{e}^{s_{\varepsilon, \mu} A} y_{\varepsilon, \mu}\right|_{X} \leq \mu \leq \frac{\delta_{0}}{4 K_{g}} .
$$

Then with (3.32), (3.31) and this inequality we have:

$$
\delta_{0}-\mu\left|x_{0}\right|_{X}^{2}=w_{\sigma}\left(t_{0}, x_{0}\right)-v_{\sigma}\left(t_{0}, x_{0}\right)-\mu\left|x_{0}\right|_{X}^{2} \leq \frac{\delta_{0}}{8}+K_{g}\left(\frac{\delta_{0}}{4 K_{g}}\right)+\frac{\delta_{0}}{8}=\frac{\delta_{0}}{2} .
$$

By passing to the limit when $\mu$ tends to zero, we obtain a contradiction. Therefore we have proved that

$$
w(t, x) \leq v(t, x) \text { for all }(t, x) \in Q_{\tau} .
$$

\section{Properties of the VAlue function and existence Results}

For all $t \in[0, T]$ and $x \in X$, we consider the optimal control problem

$\left(\mathcal{P}_{t, x}\right)$

$$
\min \{J(t, y, u) \mid u \in \mathcal{M}(t, T ; U) \text { and }(y, u) \text { is solution of equation }(2.1)\},
$$

where the cost functional $J$ is defined by

$$
J(t, y, u)=\int_{t}^{T} L(r, y(r), u(r)) \mathrm{d} r+g(y(T)) .
$$

We assume that assumptions (i)-(viii) of Section 2 are satisfied. Let $v(t, x)$ be the value function of problem $\left(\mathcal{P}_{t, x}\right)$, that is

$$
v(t, x)=\inf _{u \in \mathcal{M}(t, T ; U)} J\left(t, y_{t, x, u}, u\right) .
$$

In the following it will be convenient to use the notation

$$
I_{t, x}(u)=J\left(t, y_{t, x, u}, u\right) .
$$




\subsection{Properties of the value function}

Proposition 4.1. For all $x, x_{0} \in X$, and all $t \in[0, T]$, the value function $v$ satisfies:

$$
\left|v(t, x)-v\left(t, x_{0}\right)\right| \leq K_{v}\left|x-x_{0}\right|_{X}
$$

with $K_{v}$ independent of $t$.

Proof. With estimate (2.38), we have

$$
\begin{aligned}
v(t, x)-v\left(t, x_{0}\right) & =\inf _{u \in \mathcal{M}(t, T ; U)} I_{t, x}(u)-\inf _{u \in \mathcal{M}(t, T ; U)} I_{t, x_{0}}(u) \\
& \leq \sup _{u \in \mathcal{M}(t, T ; U)}\left(I_{t, x}(u)-I_{t, x_{0}}(u)\right) \\
& \leq \sup _{u \in \mathcal{M}(t, T ; U)}\left\{\int_{t}^{T}\left|L\left(\cdot, y_{t, x, u}, u\right)-L\left(\cdot, y_{t, x_{0}, u}, u\right)\right| \mathrm{d} r+\left|g\left(y_{t, x, u}(T)\right)-g\left(y_{t, x_{0}, u}(T)\right)\right|\right\} \\
& \leq \sup _{u \in \mathcal{M}(t, T ; U)}\left\{\int_{t}^{T} K_{L}\left|y_{t, x, u}(r)-y_{t, x_{0}, u}(r)\right|_{X} \mathrm{~d} r+K_{g}\left|y_{t, x, u}(T)-y_{t, x_{0}, u}(T)\right|_{X}\right\} \\
& \leq \sup _{u \in \mathcal{M}(t, T ; U)}\left(K_{L} T+K_{g}\right)\left\|y_{t, x, u}-y_{t, x_{0}, u}\right\|_{L^{\infty}(0, T ; X)} \\
& \leq\left(K_{L} T+K_{g}\right) C_{8}(\alpha, \beta)\left|x-x_{0}\right|_{X} .
\end{aligned}
$$

By permuting $x$ and $x_{0}$, we obtain estimate (4.1) with $K_{v}=\left(K_{L} T+K_{g}\right) C_{8}(\alpha, \beta)$.

Proposition 4.2. The value function $v$ is continuous and bounded in $[0, T] \times X$.

Proof. Let us show that $v$ is bounded. As $L$ and $g$ are bounded, we have:

$$
v(t, x)=\inf _{u \in \mathcal{M}(t, T ; U)} I_{t, x}(u) \leq M_{g}+T M_{L}=M_{v}
$$

Moreover,

$$
v(t, x) \geq-\sup _{u \in \mathcal{M}(t, T ; U)}\left|I_{t, x}(u(\cdot))\right| \geq-\left(M_{g}+T M_{L}\right)=-M_{v}
$$

Hence we have

$$
|v(t, x)| \leq M_{v} \text { for all }(t, x) \in[0, T] \times X
$$

Let $(t, x) \in[0, T] \times X$ be fixed, and first show that the function $t \mapsto v(t, x)$ is continuous. Let $0 \leq s<t$, we have:

$$
\begin{aligned}
v(t, x)-v(s, x) \leq & \sup _{u \in \mathcal{M}(t, T ; U)}\left(\int_{t}^{T}\left|L\left(\cdot, y_{t, x, u}, u\right)-L\left(\cdot, y_{s, x, u}, u\right)\right| \mathrm{d} r+\left|g\left(y_{t, x, u}(T)\right)-g\left(y_{s, x, u}(T)\right)\right|\right) \\
& +\sup _{u \in \mathcal{M}(t, T ; U)} \int_{s}^{t}\left|L\left(\cdot, y_{s, x, u}, u\right)\right| \mathrm{d} r \\
\leq & \sup _{u \in \mathcal{M}(t, T ; U)}\left(K_{L} T+K_{g}\right)\left\|y_{t, x, u}-y_{s, x, u}\right\|_{L^{\infty}(t, T ; X)}+|t-s| M_{L} .
\end{aligned}
$$

By permuting $s$ and $t$, and with Proposition 2.9 we have

$$
|v(t, x)-v(s, x)| \leq C_{6}(\alpha, \beta)\left(K_{L} T+K_{g}\right) \bar{a}(t, s, x)+|t-s| M_{L}
$$


Hence with (4.1) we obtain

$$
\left|v(t, x)-v\left(s, x_{0}\right)\right| \leq\left[C_{6}(\alpha, \beta)\left(K_{L} T+K_{g}\right) \bar{a}(t, s, x)+K_{v}\left|x-x_{0}\right|_{X}\right]+|t-s| M_{L} .
$$

The proof is complete.

Proposition 4.3. For all $t \in\left[0, T\left[\right.\right.$, and all $x, x_{0} \in X$, the value function satisfies

$$
\left|v(t, x)-v\left(t, x_{0}\right)\right| \leq C(\alpha, \beta, \theta, t)\left|(-A)^{-\theta}\left(x-x_{0}\right)\right|_{X} \quad \text { for all } \theta \in[0,1-\alpha[.
$$

The constant $C(\alpha, \beta, \theta, t)$ is explicitly given in (4.7), it blows up when $t \rightarrow T$ and when $\alpha+\theta \rightarrow 1$, but it stays bounded on all compact subset of $[0, T[$.

Proof. We have

$$
v(t, x)-v\left(t, x_{0}\right) \leq \sup _{u \in \mathcal{M}(t, T ; U)}\left\{\int_{t}^{T} K_{L}\left|y_{t, x, u}(r)-y_{t, x_{0}, u}(r)\right|_{X} \mathrm{~d} r+K_{g}\left|y_{t, x, u}(T)-y_{t, x_{0}, u}(T)\right|_{X}\right\} .
$$

With estimate (2.25), we obtain

$$
v(t, x)-v\left(t, x_{0}\right) \leq\left\{\int_{t}^{T} K_{L} C_{4}(\alpha, \beta, \theta, t ; r) \mathrm{d} r+K_{g} C_{4}(\alpha, \beta, \theta, t ; T)\right\}\left|(-A)^{-\theta}\left(x-x_{0}\right)\right|_{X},
$$

where $C_{4}(\alpha, \beta, \theta, t ; r)$ is given in $(2.28)$. As $1-(\alpha+\beta+\theta)>-1$, then function $r \mapsto(r-t)^{1-(\alpha+\beta+\theta)}$ is integrable over $(t, T)$. So, one has

$$
\begin{aligned}
\int_{t}^{T} K_{L} C_{4}(\alpha, \beta, \theta, t ; r)(r) \mathrm{d} r & =K_{L} \int_{t}^{T}\left(\frac{M_{\theta}}{(r-t)^{\theta}}+\frac{C_{3}(\alpha, \beta, \theta)}{1-(\alpha+\theta)}(r-t)^{1-(\alpha+\beta+\theta)}\right) \mathrm{d} r \\
& =K_{L}\left[\frac{M_{\theta}}{1-\theta}(T-t)^{1-\theta}+\frac{C_{3}(\alpha, \beta, \theta)}{[2-(\alpha+\beta+\theta)][1-(\alpha+\theta)]}(T-t)^{2-(\alpha+\beta+\theta)}\right]
\end{aligned}
$$

Setting

we can write

$$
C(\alpha, \beta, \theta, t)=(4.6)+K_{g} C_{4}(\alpha, \beta, \theta, t ; T)
$$

And permuting $x$ and $x_{0}$ we obtain (4.5).

$$
v(t, x)-v\left(t, x_{0}\right) \leq C(\alpha, \beta, \theta, t)\left|(-A)^{-\theta}\left(x-x_{0}\right)\right|_{X} .
$$

Proposition 4.4. For all $x \in D\left((-A)^{\frac{1}{2}}\right)$ and all $s, t \in[0, T]$ there exists a constant $C$, independent of $x$, $t$ and s, such that

$$
|v(t, x)-v(s, x)| \leq C\left(1+\left|(-A)^{\frac{1}{2}} x\right|_{X}\right)|t-s|^{\frac{1}{2}}
$$

Proof. Let us recall (4.4):

$$
|v(t, x)-v(s, x)| \leq C_{6}(\alpha, \beta)\left(K_{L} T+K_{g}\right) \bar{a}(t, s, x)+|t-s| M_{L},
$$

where $\bar{a}(t, s, x)=\left|\mathrm{e}^{|t-s| A} x-x\right|_{X}+|t-s|^{1-\beta}$. With (2.11), if $x \in D\left((-A)^{\frac{1}{2}}\right)$, we get:

$$
\left|\left(\mathrm{e}^{|t-s| A}-I\right) x\right|_{X} \leq C|t-s|^{\frac{1}{2}}\left|(-A)^{\frac{1}{2}} x\right|_{X}
$$


Since $\beta \leq 1 / 2$, we have

$$
\bar{a}(t, s, x) \leq C(\beta, T)\left(1+\left|(-A)^{\frac{1}{2}} x\right|_{X}\right)|t-s|^{\frac{1}{2}}
$$

Hence

$$
|v(t, x)-v(s, x)| \leq\left[C_{6}(\alpha, \beta)\left(K_{L} T+K_{g}+C(\beta, T)\left(1+\left|(-A)^{\frac{1}{2}} x\right|_{X}\right)\right)+M_{L} T^{\frac{1}{2}}\right]|t-s|^{\frac{1}{2}}
$$

The proof is complete.

\subsection{Existence results}

Theorem 4.5. Assume that assumptions (i)-(viii) of Section 2 hold. Then the value function $v$ is a viscosity solution of the Hamilton-Jacobi-Bellman equation (1.1) in the sense of Definition 3.2.

The proof is split into three steps:

Step 1. We show that $v$ satisfies the condition $\left(\alpha_{1}\right)$ in the definition of subsolutions.

Step 2. We show that $v$ satisfies condition $\left(\alpha_{2}\right)$ in the definition of supersolutions.

Step 3. We show that $v$ satisfies both terminal conditions $\left(\beta_{1}\right)$ and $\left(\beta_{2}\right)$.

Proof. We only treat the case $0 \leq \beta<\frac{1}{2}$. The case $\beta=\frac{1}{2}$ can be treated with obvious modifications by using estimate (2.20) in place of (2.19).

Step 1. Let $\Phi \in C_{A}^{1}(] 0, T[\times X)$, and $(t, x) \in(] 0, T\left[\times D\left((-A)^{\frac{1}{2}}\right)\right) \cap \arg \max _{0, T[\times X}(v-\Phi)$. Let $u(\cdot)=u \in U$ be a constant control. For all $s>t$ we have

$$
v(t, x)-\Phi(t, x)=\max (v-\Phi) \geq v\left(s, y_{t, x, u}(s)\right)-\Phi\left(s, y_{t, x, u}(s)\right)
$$

By the dynamic programming principle it yields

$$
v(t, x) \leq \int_{t}^{s} L\left(r, y_{t, x, \tilde{u}}(r), u\right) \mathrm{d} r+v\left(s, y_{t, x, \tilde{u}}(s)\right), \quad \text { for all } \tilde{u} \in U .
$$

This inequality holds true in particular for $u$. From (4.9) we deduce

$$
\Phi(t, x)-\Phi\left(s, y_{t, x, u}(s)\right) \leq v(t, x)-v\left(s, y_{t, x, u}(s)\right) \leq \int_{t}^{s} L\left(r, y_{t, x, u}(r), u\right) \mathrm{d} r
$$

hence

$$
\Phi(t, x)-\Phi(s, x)+\Phi(s, x)-\Phi\left(s, y_{t, x, u}(s)\right)-\int_{t}^{s} L\left(r, y_{t, x, u}(r), u\right) \mathrm{d} r \leq 0 .
$$

With assumption (viii) of Section 2 satisfied by $L$ and a classical calculation we obtain:

$$
\lim _{s \searrow t}\left\{\frac{\Phi(t, x)-\Phi(s, x)}{s-t}-\frac{1}{s-t} \int_{t}^{s} L\left(r, y_{t, x, u}(r), u\right) \mathrm{d} r\right\}=-\frac{\partial \Phi}{\partial t}(t, x)-L(t, x, u) .
$$

On the other hand,

$$
\frac{\Phi(s, x)-\Phi\left(s, y_{t, x, u}(s)\right)}{s-t}=\frac{\Phi(s, x)-\Phi\left(s, \mathrm{e}^{(s-t) A} x\right)}{s-t}+\frac{\Phi\left(s, \mathrm{e}^{(s-t) A} x\right)-\Phi\left(s, y_{t, x, u}(s)\right)}{s-t}
$$


So,

$$
\begin{aligned}
\frac{\Phi\left(s, \mathrm{e}^{(s-t) A} x\right)-\Phi\left(s, y_{t, x, u}(s)\right)}{s-t}= & \frac{1}{s-t}\left(D_{x} \Phi(s, \xi(s)) \mid \mathrm{e}^{(s-t) A} x-y_{t, x, u}(s)\right)_{X} \\
= & \frac{1}{s-t}\left(D_{x} \Phi\left(s, \mathrm{e}^{(s-t) A} x\right) \mid \mathrm{e}^{(s-t) A} x-y_{t, x, u}(s)\right)_{X} \\
& +\frac{1}{s-t}\left(D_{x} \Phi(s, \xi(s))-D_{x} \Phi\left(s, \mathrm{e}^{(s-t) A} x\right) \mid \mathrm{e}^{(s-t) A} x-y_{t, x, u}(s)\right)_{X},
\end{aligned}
$$

where

$$
\xi(s)=\lambda(s) \mathrm{e}^{(s-t) A} x+(1-\lambda(s)) y_{t, x, u}(s),
$$

for a function $\lambda:[t, T] \rightarrow] 0,1\left[\right.$. We denote by $K_{\Phi}$ the Lipschitz constant of $D_{x} \Phi(t, \cdot)$ in $X$ (condition $(\beta)$ in the definition of $\left.C_{A}^{1}(] 0, T[\times X)\right)$. With estimate (2.19), we have

$$
\begin{aligned}
\mid \frac{1}{s-t}\left(D_{x} \Phi(s, \xi(s)\right. & \left.-D_{x} \Phi\left(s, \mathrm{e}^{(s-t) A} x\right) \mid \mathrm{e}^{(s-t) A} x-y_{t, x, u}(s)\right)_{X} \mid \\
\leq & \frac{1}{s-t} K_{\Phi}\left|\xi(s)-\mathrm{e}^{(s-t) A} x\right|_{X}\left|\mathrm{e}^{(s-t) A} x-y_{t, x, u}(s)\right|_{X} \\
& =\frac{1}{s-t} K_{\Phi}(1-\lambda(s))\left|\mathrm{e}^{(s-t) A} x-y_{t, x, u}(s)\right|_{X}^{2} \\
& \leq \frac{1}{s-t} K_{\Phi}\left|\mathrm{e}^{(s-t) A} x-y_{t, x, u}(s)\right|_{X}^{2} \\
\leq & C_{1}(\beta) K_{\Phi} \frac{|s-t|^{2(1-\beta)}}{s-t}=C|s-t|^{1-2 \beta} \rightarrow 0 \text { when } s \searrow t, \text { as } \beta<1 / 2,
\end{aligned}
$$

and $C$ is independent of $u$ ( $C$ depends only on $M_{U}$ ). In the case when $\beta=\frac{1}{2}$, using (2.20) we obtain $C_{1}\left(\beta,|x|_{D\left((-A)^{\frac{1}{2}}\right)}\right) K_{\Phi}|s-t|^{2 \beta_{0}}$ in place of $C|s-t|^{1-2 \beta}$. For the term (4.13), we have

$$
\begin{aligned}
\frac{1}{s-t}\left(D_{x} \Phi\left(s, \mathrm{e}^{(s-t) A} x\right) \mid \mathrm{e}^{(s-t) A} x-y_{t, x, u}(s)\right)_{X} & \\
& =\left((-A)^{\beta} D_{x} \Phi\left(s, \mathrm{e}^{(s-t) A} x\right) \mid \frac{-1}{s-t} \int_{t}^{s} \mathrm{e}^{(s-r) A}\left[B u-F\left(r, \Lambda y_{t, x, u}(r)\right)\right] \mathrm{d} r\right)_{X}
\end{aligned}
$$

We know that $\mathrm{e}^{(s-t) A} x$ tends to $x$ in $D\left((-A)^{\frac{1}{2}}\right)$ when $s \searrow t$. Thus using condition $(\delta)$ in the definition of $C_{A}^{1}(] 0, T[\times X)$ and Proposition 2.2, we can pass to the limit in (4.15), when $s \searrow t$, and we obtain

$$
\lim _{s \searrow t} \frac{\Phi\left(s, \mathrm{e}^{(s-t) A} x\right)-\Phi\left(s, y_{t, x, u}(s)\right)}{s-t}=-\left((-A)^{\beta} D_{x} \Phi(t, x) \mid B u-F(t, \Lambda x)\right)_{X} .
$$

Moreover

$$
\frac{\Phi(s, x)-\Phi\left(s, \mathrm{e}^{(s-t) A} x\right)}{s-t}=\left(D_{x} \Phi(s, \eta(s)) \mid \frac{x-\mathrm{e}^{(s-t) A} x}{s-t}\right)_{X}
$$

where

$$
\eta(s)=\lambda(s) \mathrm{e}^{(s-t) A} x+(1-\lambda(s)) x,
$$


for a fonction $\lambda:[t, T] \rightarrow] 0,1\left[\right.$. We remark that $\eta(s) \in D\left((-A)^{\frac{1}{2}}\right)$ as $x \in D\left((-A)^{\frac{1}{2}}\right)$. Thus $D_{x} \Phi(s, \eta(s)) \in$ $D\left((-A)^{\frac{1}{2}}\right)$ due to $(\gamma)$ in the definition of $C_{A}^{1}(] 0, T[\times X)$. The right hand side of (4.17) can be written as follows

$$
\begin{aligned}
\left(D_{x} \Phi(s, \eta(s)) \mid \frac{-1}{s-t} \int_{0}^{s-t} A \mathrm{e}^{r A} x \mathrm{~d} r\right)_{X} & =\frac{1}{s-t} \int_{0}^{s-t}\left((-A)^{\frac{1}{2}} D_{x} \Phi(s, \eta(s)) \mid(-A)^{\frac{1}{2}} \mathrm{e}^{r A} x\right)_{X} \mathrm{~d} r \\
& \rightarrow\left((-A)^{\frac{1}{2}} D_{x} \Phi(t, x) \mid(-A)^{\frac{1}{2}} x\right)_{X} \quad \text { when } s \searrow t,
\end{aligned}
$$

because the mapping $(s, x) \mapsto D_{x} \Phi(s, x)$ is continuous from $[0, T] \times D\left((-A)^{\frac{1}{2}}\right)$ into $D\left((-A)^{\frac{1}{2}}\right)$. Hence with (4.10), (4.11), (4.12), (4.16) and (4.19), we conclude that

$$
\begin{aligned}
0 \geq & -\frac{\partial \Phi}{\partial t}(t, x)-L(t, x, u)+\left((-A)^{\beta} D_{x} \Phi(t, x) \mid F(t, \Lambda x)\right)_{X} \\
& -\left((-A)^{\beta} D_{x} \Phi(t, x) \mid B u\right)_{X}+\left((-A)^{\frac{1}{2}} D_{x} \Phi(t, x) \mid(-A)^{\frac{1}{2}} x\right)_{X} .
\end{aligned}
$$

By passing to the supremum with respect to $u \in U$ we have:

$$
\begin{aligned}
0 \geq & -\frac{\partial \Phi}{\partial t}(t, x)+\left((-A)^{\frac{1}{2}} D_{x} \Phi(t, x) \mid(-A)^{\frac{1}{2}} x\right)_{X} \\
& +\left((-A)^{\beta} D_{x} \Phi(t, x) \mid F(t, \Lambda x)\right)_{X}+H\left(t, x(-A)^{\beta} D_{x} \Phi(t, x)\right) .
\end{aligned}
$$

Thus the condition $\left(\alpha_{1}\right)$ in the definition of subsolutions of equation (1.1) is satisfied.

Step 2. Let $\Phi$ be in $C_{A}^{1}(] 0, T[\times X)$, and $\left.(t, x) \in\right] 0, T\left[\times D\left((-A)^{\frac{1}{2}}\right) \cap \arg \min (v-\Phi)\right.$. For all $u(\cdot) \in \mathcal{M}(0, T ; U)$ we have

$$
v(t, x)-v\left(s, y_{t, x, u}(s)\right) \leq \Phi(t, x)-\Phi\left(s, y_{t, x, u}(s)\right) .
$$

Thanks to the dynamic programming principle, for all $\varepsilon>0$, there exists a control $u_{\varepsilon}(\cdot), \varepsilon$-optimal, such that

$$
\varepsilon(s-t)+v(t, x) \geq \int_{t}^{s} L\left(r, y_{t, x, u_{\varepsilon}}(r), u_{\varepsilon}(r)\right) \mathrm{d} r+v\left(s, y_{t, x, u_{\varepsilon}}(s)\right) .
$$

Setting $u=u_{\varepsilon}$ in (4.20), and substracting (4.21), we obtain:

$$
\Phi(t, x)-\Phi\left(s, y_{t, x, u_{\varepsilon}}(s)\right)+\varepsilon(s-t)-\int_{t}^{s} L\left(r, y_{t, x, u_{\varepsilon}}(r), u_{\varepsilon}(r)\right) \mathrm{d} r \geq 0,
$$

that is:

$$
\frac{\Phi(t, x)-\Phi\left(s, y_{t, x, u_{\varepsilon}}(s)\right)}{s-t}+\varepsilon-\frac{1}{s-t} \int_{t}^{s} L\left(r, y_{t, x, u_{\varepsilon}}(r), u_{\varepsilon}(r)\right) \mathrm{d} r \geq 0
$$

There exist a function $\eta_{1}:[0, T-t] \mapsto \mathbb{R}$, a function $\lambda_{2}:[t, T] \mapsto[0,1]$, and a function $\lambda_{3}:[t, T] \mapsto[0,1]$ such that

$$
\begin{aligned}
\frac{\Phi(t, x)-\Phi\left(s, y_{t, x, u_{\varepsilon}}(s)\right)}{s-t}= & \frac{\Phi(t, x)-\Phi(s, x)}{s-t}+\frac{\Phi(s, x)-\Phi\left(s, \mathrm{e}^{(s-t) A} x\right)}{s-t}+\frac{\Phi\left(s, \mathrm{e}^{(s-t) A} x\right)-\Phi\left(s, y_{t, x, u_{\varepsilon}}(s)\right)}{s-t} \\
= & -\frac{\partial \Phi}{\partial t}(t, x)+\eta_{1}(s-t)+\left(D_{x} \Phi\left(s, \eta_{2}(s)\right) \mid \frac{x-\mathrm{e}^{(s-t) A} x}{s-t}\right)_{X} \\
& +\frac{1}{s-t}\left(D_{x} \Phi\left(s, \mathrm{e}^{(s-t) A} x\right) \mid \mathrm{e}^{(s-t) A} x-y_{t, x, u_{\varepsilon}}(s)\right)_{X} \\
& +\frac{1}{s-t}\left(D_{x} \Phi\left(s, \eta_{3}(s)\right)-D_{x} \Phi\left(s, \mathrm{e}^{(s-t) A} x\right) \mid \mathrm{e}^{(s-t) A} x-y_{t, x, u_{\varepsilon}}(s)\right)_{X}
\end{aligned}
$$


where

As in (4.14) we have

$$
\begin{aligned}
& \eta_{1}(s-t) \rightarrow 0 \quad \text { when } \quad s \searrow t, \quad \eta_{2}(s)=\lambda_{2}(s) \mathrm{e}^{(s-t) A} x+\left(1-\lambda_{2}(s)\right) x, \\
& \eta_{3}(s)=\lambda_{3}(s) \mathrm{e}^{(s-t) A} x+\left(1-\lambda_{3}(s)\right) y_{t, x, u_{\varepsilon}}(s) .
\end{aligned}
$$

$$
\frac{1}{s-t}\left|\left(D_{x} \Phi\left(s, \eta_{3}(s)\right)-D_{x} \Phi\left(s, \mathrm{e}^{(s-t) A} x\right) \mid \mathrm{e}^{(s-t) A} x-y_{t, x, u_{\varepsilon}}(s)\right)_{X}\right| \leq C_{1}(\beta) K_{\Phi}|s-t|^{1-2 \beta} \rightarrow 0 \text { when } s \searrow t .
$$

In (4.19) we have shown that

$$
\left(D_{x} \Phi\left(s, \eta_{2}(s)\right) \mid \frac{x-\mathrm{e}^{(s-t) A} x}{s-t}\right)_{X}=\left((-A)^{\frac{1}{2}} D_{x} \Phi(t, x) \mid(-A)^{\frac{1}{2}} x\right)_{X}+\eta_{4}(s-t),
$$

where $\eta_{4}(s-t) \rightarrow 0$ when $s \searrow t$.

Due to the definition of $H$, we can use the inequality

$$
\begin{aligned}
-\left((-A)^{\beta} \mathrm{e}^{(s-r) A} D_{x} \Phi\left(s, \mathrm{e}^{(s-t) A} x\right) \mid B u_{\varepsilon}(r)\right)-L\left(r, y_{t, x, u_{\varepsilon}}(r), u_{\varepsilon}(r)\right) & \\
& \leq H\left(r, y_{t, x, u_{\varepsilon}}(r),(-A)^{\beta} \mathrm{e}^{(s-r) A} D_{x} \Phi\left(s, \mathrm{e}^{(s-t) A} x\right)\right) .
\end{aligned}
$$

Thus

$$
\begin{aligned}
& \frac{1}{s-t}\left(D_{x} \Phi\left(s, \mathrm{e}^{(s-t) A} x\right) \mid \mathrm{e}^{(s-t) A} x-y_{t, x, u_{\varepsilon}}(s)\right) \\
&=\left(D_{x} \Phi\left(s, \mathrm{e}^{(s-t) A} x\right) \mid \frac{-1}{s-t} \int_{t}^{s}(-A)^{\beta} \mathrm{e}^{(s-r) A}\left[B u_{\varepsilon}(r)-F\left(r, \Lambda y_{t, x, u_{\varepsilon}}(r)\right)\right] \mathrm{d} r\right) \\
& \leq \frac{1}{s-t} \int_{t}^{s}\left[H\left(r, y_{t, x, u_{\varepsilon}}(r),(-A)^{\beta} \mathrm{e}^{(s-r) A} D_{x} \Phi\left(s, \mathrm{e}^{(s-t) A} x\right)\right)+L\left(r, y_{t, x, u_{\varepsilon}}(r), u_{\varepsilon}(r)\right)\right] \mathrm{d} r \\
&+\frac{1}{s-t} \int_{t}^{s}\left((-A)^{\beta} \mathrm{e}^{(s-r) A} D_{x} \Phi\left(s, \mathrm{e}^{(s-t) A} x\right) \mid F\left(r, \Lambda y_{t, x, u_{\varepsilon}}(r)\right)\right) \mathrm{d} r .
\end{aligned}
$$

By collecting together all the terms in (4.22), we obtain

$$
\begin{aligned}
\varepsilon+\frac{1}{s-t} \int_{t}^{s} H\left(r, y_{t, x, u_{\varepsilon}}(r),(-A)^{\beta} \mathrm{e}^{(s-r) A} D_{x} \Phi\left(s, \mathrm{e}^{(s-t) A} x\right)\right) \\
\quad-\frac{\partial \Phi}{\partial t}(t, x)+\eta_{1}(s-t)+\eta_{4}(s-t)+\left((-A)^{\frac{1}{2}} D_{x} \Phi(t, x) \mid(-A)^{\frac{1}{2}} x\right)_{X} \\
\quad+\frac{1}{s-t} \int_{t}^{s}\left((-A)^{\beta} \mathrm{e}^{(s-r) A} D_{x} \Phi\left(s, \mathrm{e}^{(s-t) A} x\right) \mid F\left(r, \Lambda y_{t, x, u_{\varepsilon}}(r)\right)\right)_{X} \\
\geq-C_{1}(\beta) K_{\Phi}|s-t|^{1-2 \beta} .
\end{aligned}
$$

(If $\beta=\frac{1}{2}$, we have to replace the last line by $-C_{1}\left(\beta,|x|_{D\left((-A)^{\frac{1}{2}}\right)}\right) K_{\Phi}|s-t|^{1-2 \beta_{0}}$.) With Proposition 2.2 we have

$$
\Lambda y_{t, x, u_{\varepsilon}}(r) \rightarrow \Lambda x \text { uniformly w.r. to } u_{\varepsilon} \text { when } r \rightarrow t .
$$

By passing to the limit in the previous inequality when $s \rightarrow t$, and after when $\varepsilon \rightarrow 0$, we obtain:

$$
\begin{aligned}
0 \leq & -\frac{\partial \Phi}{\partial t}(t, x)+\left((-A)^{\frac{1}{2}} D_{x} \Phi(t, x) \mid(-A)^{\frac{1}{2}} x\right)_{X} \\
& +H\left(t, x(-A)^{\beta} D_{x} \Phi(t, x)\right)+\left((-A)^{\beta} D_{x} \Phi(t, x) \mid F(t, \Lambda x)\right)_{X} .
\end{aligned}
$$


Step 3. Let us show that

$$
\lim _{t \searrow 0} \sup _{x \in X}\left|v(T-t, x)-g\left(\mathrm{e}^{t A} x\right)\right|=0 .
$$

From the definition of $v(T-t, x)$, it follows that

$$
v(T-t, x)-g\left(\mathrm{e}^{t A} x\right) \leq \int_{T-t}^{T} L\left(r, y_{T-t, x, u}(r), u(r)\right) \mathrm{d} r+K_{g}\left|y_{T-t, x, u}(T)-\mathrm{e}^{t A} x\right|_{X},
$$

for all $u \in \mathcal{M}(T-t, T ; U)$. Due to estimate (2.19), there exists a constant $C$ independent of $t$ and $u$ such that

$$
\left|y_{T-t, x, u}(T)-\mathrm{e}^{t A} x\right| \leq C t^{1-\beta} .
$$

Then we have

$$
v(T-t, x)-g\left(\mathrm{e}^{t A} x\right) \leq \int_{T-t}^{T} L\left(r, y_{T-t, x, u}(r), u(r)\right) \mathrm{d} r+K_{g} C t^{1-\beta}
$$

for all $u(\cdot) \in \mathcal{M}(T-t, T ; U)$. Since $L$ is bounded we obtain

$$
\lim _{t \searrow 0} \sup _{x \in X}\left[v(T-t, x)-g\left(\mathrm{e}^{t A} x\right)\right] \leq 0 .
$$

For the opposite inequality, we choose a control $u_{\delta}(\cdot) \in \mathcal{M}(T-t, T ; U), \delta$-optimal, such that

$$
v(T-t, x)+\delta>\int_{T-t}^{T} L\left(r, y_{\delta}(r), u_{\delta}(r)\right) \mathrm{d} r+g\left(y_{\delta}(T)\right),
$$

where $y_{\delta}=y_{T-t, x, u_{\delta}}$. As $L$ is bounded, for $t$ small enough we have

$$
v(T-t, x)+\delta>-\delta+g\left(y_{\delta}(T)\right) .
$$

We can write

and

$$
g\left(y_{\delta}(T)\right) \geq-\left|g\left(y_{\delta}(T)\right)-g\left(\mathrm{e}^{t A} x\right)\right|+g\left(\mathrm{e}^{t A} x\right)
$$

Hence

$$
v(T-t, x)-g\left(\mathrm{e}^{t A} x\right)>-2 \delta-K_{g}\left|y_{\delta}(T)-\mathrm{e}^{t A} x\right|_{X} \geq-2 \delta-K_{g} C t^{1-\beta} .
$$

$$
\lim _{t \searrow 0} \sup _{x \in X}\left[v(T-t, x)-g\left(\mathrm{e}^{t A} x\right)\right] \geq-2 \delta .
$$

We can pass to the limit when $\delta$ tends to zero, and we have shown that conditions $\left(\beta_{1}\right)$ and $\left(\beta_{2}\right)$ of Definition 3.2 are satisfied by $v$.

\section{Examples of optimal CONTROl Problems}

In this section we study the value function of problems of the form

$\left(\widehat{\mathcal{P}}_{t, x}\right)$

$$
\min \{\widehat{J}(t, u, y) \mid u \in \mathcal{M}(t, T ; U) \text { and }(y, u) \text { is solution of equation (5.1) }\} \text {. }
$$

where

and

$$
y^{\prime}=A y+(-A)^{\beta}[B u-\widehat{F}(\cdot, \Lambda y)] \quad \text { in }(t, T), \quad y(t)=x,
$$

$$
\widehat{J}(t, y, u)=\int_{t}^{T} \widehat{L}(r, y(r), u(r)) \mathrm{d} r+\widehat{g}(y(T))
$$


We do not assume that $\widehat{F}, \widehat{L}$ and $\widehat{g}$ obeys the assumptions of Section 2. But we consider examples such that, for all $u \in \mathcal{M}(t, T ; U)$, and all $x \in Y$ - where $Y$ is a suitably chosen Banach space - equation (5.1) admits a unique solution which satisfies

$$
\|y\|_{L_{w}^{\infty}(0, T ; Y)} \leq R\left(M_{0}, T\right) \quad \text { and } \quad\|\Lambda y\|_{L_{w}^{\infty}\left(0, T ; Y_{0}\right)} \leq R\left(M_{0}, T\right) \quad \text { if }|x|_{Y} \leq M_{0}
$$

for all $M_{0}>0$, where $R\left(M_{0}, T\right)$ is a function of $M_{0}$ and $T, Y_{0}$ is a subspace of $X_{0}$ such that $\Lambda$ is continuous from $D\left((-A)^{\alpha}\right) \cap Y$ into $Y_{0}$. We denote by $\widehat{v}(t, x)$ the value function of problem $\left(\widehat{\mathcal{P}}_{t, x}\right)$. We introduce a projection operator $P_{M_{0}}$ from $X$ on the ball $B_{Y}\left(R\left(M_{0}, T\right)\right)$ in $Y$, centered at the origin and with radius $R\left(M_{0}, T\right)$, and a projection operator $P_{M_{0}}^{0}$ from $X_{0}$ on the ball $B_{Y_{0}}\left(R\left(M_{0}, T\right)\right)$ in $Y_{0}$, centered at the origin and with radius $R\left(M_{0}, T\right)$. We set

$$
F_{M_{0}}(\cdot, \Lambda y)=\widehat{F}\left(\cdot, P_{M_{0}}^{0} \Lambda y\right), \quad L_{M_{0}}(r, y, u)=\widehat{L}\left(r, P_{M_{0}} y, u\right), \quad \text { and } \quad g_{M_{0}}(y)=\widehat{g}\left(P_{M_{0}} y\right) .
$$

In the different examples we verify that $F_{M_{0}}, L_{M_{0}}$, and $g_{M_{0}}$ obeys the assumptions of Section 2 (with constants depending on $\left.M_{0}\right)$. We denote by $\left(\mathcal{P}_{t, x}^{M_{0}}\right)$ the problem $\left(\mathcal{P}_{t, x}\right)$ of Section 1, corresponding to $F_{M_{0}}, L_{M_{0}}$, and $g_{M_{0}}$, and by $v_{M_{0}}(t, x)$ its value function. We verify that

$$
\widehat{v}(t, x)=v_{M_{0}}(t, x) \quad \text { for all } x \in Y \quad \text { such that }|x|_{Y} \leq M_{0} .
$$

Due to Theorems 3.5 and 4.5, we know that $v_{M_{0}}$ is the unique viscosity solution of equation (1.1) corresponding to $F_{M_{0}}, L_{M_{0}}$, and $g_{M_{0}}$. Due to the definition of problem $\left(\mathcal{P}_{t, x}^{M_{0}}\right)$, it is obvious that

$$
v_{M_{1}}(t, x)=v_{M_{0}}(t, x) \quad \text { for all } x \in Y \quad \text { such that }|x|_{Y} \leq M_{0},
$$

if $M_{1} \geq M_{0}$. Thus, to characterize $\widehat{v}(t, x)$ when $x \in Y$, it is enough to characterize $v_{M_{0}}(t, x)$ for all $M_{0}$. And $v_{M_{0}}(t, x)$ is characterized as the unique viscosity solution of equation (1.1) corresponding to $F_{M_{0}}, L_{M_{0}}$, and $g_{M_{0}}$.

In the following $\Omega$ is a bounded open subset in $\mathbb{R}^{N}$, with a regular boundary $\Gamma$, and we set $\left.\Omega_{t, T}=\right] t, T[\times \Omega$ and $\left.\Sigma_{t, T}=\right] t, T[\times \Gamma$.

\subsection{State equation of example 1}

Consider the equation

$$
\frac{\partial y}{\partial t}-\Delta y+y=f \quad \text { in } \Omega_{t, T}, \quad \frac{\partial y}{\partial n}+\widehat{h}(y)=u \quad \text { on } \Sigma_{t, T}, \quad y(t)=x_{0} \quad \text { in } \Omega,
$$

where $\widehat{h}$ is a regular nondecreasing function satisfying $h(y)=0$ (e.g. the well known 'Stefan-Boltzmann radiation condition' corresponds to $\widehat{h}(y)=k_{r}|y|^{3} y+k_{c} y, k_{r}$ is the radiation coefficient and $k_{c}$ the convection coefficient [23]). We make the following assumptions.

$\left(\mathcal{A}_{1}\right) U$ is a closed bounded convex and nonempty subset in $L^{q}(\Gamma)$ for some $q \geq 2$, and it obeys the condition

$$
q>N-1, \quad|u|_{L^{2}(\Gamma)} \leq M_{U} \quad \text { and } \quad|u|_{L^{q}(\Gamma)} \leq M_{U} \quad \text { for all } u \in U .
$$

$\left(\mathcal{A}_{2}\right)$ The function $f$ belongs to $C^{0, \eta_{1}}\left([0, T] ; L^{p}(\Omega)\right)$ for some $p \geq 2$, and it obeys the condition

$$
p \geq N / 2, \quad\|f\|_{C\left([0, T] ; L^{p}(\Omega)\right)} \leq M_{f}, \quad \text { and } \quad\|f\|_{C\left([0, T] ; L^{2}(\Omega)\right)} \leq M_{f} .
$$


Let be $X=L^{2}(\Omega), X_{\Gamma}=L^{2}(\Gamma), X_{0}=L^{2}(\Gamma)$, and let us define the unbounded operator $A$ in $X$ by

$$
D(A)=\left\{x \in H^{2}(\Omega) \mid \frac{\partial x}{\partial n}=0 \text { on } \Gamma\right\} \quad \text { and } \quad A x=\Delta x-x \text { for all } x \in D(A) .
$$

Assumption (i) of Section 2 is clearly satisfied. We define the Neumann operator $N \in \mathcal{L}\left(X_{\Gamma} ; X\right)$ by $N u=z$, where $z$ is the solution of the boundary value problem

$$
-\Delta z+z=0 \quad \text { in } \Omega, \quad \frac{\partial z}{\partial n}=u \quad \text { on } \Gamma .
$$

The operator $N$ is also bounded from $X_{\Gamma}$ into $H^{3 / 2}(\Omega)$, and from $X_{\Gamma}$ into $D\left((-A)^{\alpha}\right)$ for all $0<\alpha<\frac{3}{4}$ (see [21]). With the extrapolation method, the semigroup $\left(\mathrm{e}^{t A}\right)_{t \geq 0}$ can be extended to $\left(D\left(A^{*}\right)\right)^{\prime}=(D(A))^{\prime}$. Denoting by $\left(\mathrm{e}^{t \widehat{A}}\right)_{t \geq 0}$ the corresponding semigroup, $(\widehat{A}, D(\widehat{A}))$, its infinitesimal generator, is an unbounded operator in $\left(D\left(A^{*}\right)\right)^{\prime}$ with domain $D(\widehat{A})=X$. Thus the operator $(-\widehat{A}) N$ is bounded from $X_{\Gamma}$ into $\left(D\left(A^{*}\right)\right)^{\prime}$. The operator $\Lambda$ is the trace operator. It satisfies assumption (iii) of Section 2 for all $\alpha \in] \frac{1}{4}, \frac{1}{2}$ [. It is well known - see e.g. [4] - that equation (5.2) can be rewritten in the form

$$
y^{\prime}=\widehat{A} y+f+(-\widehat{A}) N[u-\widehat{h}(\Lambda y)] \quad \text { in }(t, T), \quad y(t)=x_{0} .
$$

We define the operator $B$ by $B=(-\widehat{A})^{1-\beta} N$ for some $\beta$ given fixed in $] 1 / 4,1 / 2[$. Due to the regularizing properties of $N$, mentioned above, we can also verify that $B \in \mathcal{L}\left(X_{\Gamma}, X\right)$. We set $\widehat{F}(t, y)=(-A)^{-\beta} f(t)+B \widehat{h}(y)$. Equation (5.3) is nothing else than

$$
y^{\prime}=\widehat{A} y+(-\widehat{A})^{\beta}[B u-\widehat{F}(\Lambda y)] \quad \text { in }(t, T), \quad y(t)=x_{0} .
$$

As in [24], Theorem 3.1, we can prove the following result.

Theorem 5.1. For all $x_{0} \in L^{\infty}(\Omega)$ and all $u \in \mathcal{M}(t, T ; U)$, equation (5.2) admits a unique weak solution in $C\left([t, T] ; L^{2}(\Omega)\right)$. This solution belongs to $\left.\left.C_{b}(] t, T\right] \times \bar{\Omega}\right)$ and it satisfies the estimate

$$
\|y\|_{L^{\infty}\left(\Omega_{t, T}\right)}+\|y\|_{L^{\infty}\left(\Sigma_{t, T}\right)} \leq\left|x_{0}\right|_{L^{\infty}(\Omega)}+C(p, \tilde{p}, q, \tilde{q}, \Omega, T)\left(\|f\|_{L^{\tilde{p}}\left(t, T ; L^{p}(\Omega)\right)}+\|u\|_{L^{\tilde{q}}\left(t, T ; L^{q}(\Gamma)\right)}\right),
$$

where the exponents $1<\tilde{p}<\infty$ and $1<\tilde{q}<\infty$ obeys

$$
\frac{N}{2 p}+\frac{1}{\tilde{p}}<1 \quad \text { and } \quad \frac{N-1}{2 q}+\frac{1}{\tilde{q}}<\frac{1}{2}
$$

and the constant $C(p, \tilde{p}, q, \tilde{q}, \Omega, T)$ depends on $N, p, \tilde{p}, q, \tilde{q}, \Omega, T$, but is independent of $t$.

From Theorem 5.1, and assumptions $\left(\mathcal{A}_{1}\right)$ and $\left(\mathcal{A}_{2}\right)$, we deduce

$$
\|y\|_{L^{\infty}\left(\Omega_{t, T}\right)} \leq\left|x_{0}\right|_{L^{\infty}(\Omega)}+C(p, \tilde{p}, q, \tilde{q}, \Omega, T)\left((T-t)^{1 / \tilde{p}} M_{f}+(T-t)^{1 / \tilde{q}} M_{U}\right) .
$$

\subsection{Setting of the control problem for example 1}

For all $t \in\left[0, T\left[\right.\right.$, and all $x_{0} \in L^{\infty}(\Omega)$, we study the following control problem

$\left(\widehat{\mathcal{P}}_{t, x_{0}}\right)$ $\min \{\widehat{J}(t, u, y) \mid u \in \mathcal{M}(t, T ; U)$ and $(y, u)$ is solution of equation $(5.2)\}$, 
where the cost function $\widehat{J}$ is of the form

$$
\widehat{J}(t, u, y)=\int_{t}^{T} \int_{\Omega} \widehat{G}(s, \xi, y(s, \xi)) \mathrm{d} \xi \mathrm{d} s+\int_{t}^{T} \int_{\Gamma} \widehat{K}(s, \sigma, u(s, \sigma)) \mathrm{d} \sigma \mathrm{d} s+\int_{\Omega} \widehat{k}(\xi, y(T, \xi)) \mathrm{d} \xi .
$$

We make the following assumptions on the data of problem $\left(\widehat{\mathcal{P}}_{t, x_{0}}\right)$.

$\left(\mathcal{A}_{3}\right)$ For all $(t, y) \in[0, T] \times \mathbb{R}, \widehat{G}(t, \cdot, y)$ is measurable in $\Omega$. For almost all $\xi \in \Omega, \widehat{G}(\cdot, \xi, \cdot)$ is continuous in $[0, T] \times \mathbb{R}$, and we have the estimates:

$$
\begin{aligned}
& C_{\widehat{G}} \leq \widehat{G}(t, \xi, y) \leq \widehat{G}_{1}(\xi) \times \eta(|y|), \\
& |\widehat{G}(t, \xi, y)-\widehat{G}(s, \xi, z)| \leq \widehat{G}_{2}(\xi)(\eta(|y|)+\eta(|z|))\left(|t-s|^{\eta_{2}}+|y-z|\right),
\end{aligned}
$$

where $\widehat{G}_{1} \in L^{1}(\Omega), \widehat{G}_{2} \in L^{2}(\Omega)$, and $\eta$ an increasing function from $\mathbb{R}^{+}$in $\mathbb{R}^{+}$.

$\left(\mathcal{A}_{4}\right)$ For all $(t, u) \in \mathbb{R}^{2}, \widehat{K}(t, \cdot, u)$ is measurable in $\Gamma$. For a.e. $(t, \sigma) \in \Sigma, \widehat{K}(t, \sigma, \cdot)$ is convex. For a.e. $\sigma \in \Gamma$, $\widehat{K}(\cdot, \sigma, \cdot)$ is continuous in $\mathbb{R}^{2}$ and we have the estimates:

$C_{\widehat{K}} \leq \widehat{K}(t, \sigma, u) \leq \widehat{K}_{1}(\sigma)+c_{0}|u|^{q} \quad$ and $\quad|\widehat{K}(t, \sigma, u)-\widehat{K}(s, \sigma, u)| \leq\left(\widehat{K}_{1}(\sigma)+c_{0}|u|^{q}\right)|t-s|^{\eta_{2}}$,

where $\widehat{K}_{1} \in L^{1}(\Gamma)$.

$\left(\mathcal{A}_{5}\right)$ For all $y \in \mathbb{R}, \widehat{k}(\cdot, y)$ is measurable in $\Omega$. For a.e. $\xi \in \Omega, \widehat{k}(\xi, \cdot)$ is continuous in $\mathbb{R}$ and we have the estimates:

$$
C_{\widehat{k}} \leq \widehat{k}(\xi, y) \leq \widehat{k}_{1}(\xi) \times \eta(|y|) \quad \text { and } \quad|\widehat{k}(\xi, y)-\widehat{k}(\xi, z)| \leq \widehat{k}_{2}(\xi)(\eta(|y|)+\eta(|z|))|y-z|,
$$

where $\widehat{k}_{1} \in L^{1}(\Omega), \widehat{k}_{2} \in L^{2}(\Omega)$, and $\eta$ as in $\left(\mathcal{A}_{1}\right)$.

The value function of problem $\left(\widehat{\mathcal{P}}_{t, x_{0}}\right)$ is defined by

$$
\widehat{v}\left(t, x_{0}\right)=\inf _{\mathcal{M}(t, T ; U)} \widehat{J}\left(t, \widehat{y}_{t, x_{0}, u}, u\right),
$$

where $\widehat{y}_{t, x_{0}, u}$ is the solution of equation (5.2).

\subsection{Existence of solution to $\left(\widehat{\mathcal{P}}_{t, x_{0}}\right)$}

We have the following theorem [23, Th. 6.1].

Theorem 5.2. For all $t \in\left[0, T\left[\right.\right.$, all $x_{0} \in L^{\infty}(\Omega)$, problem $\left(\widehat{\mathcal{P}}_{t, x_{0}}\right)$ admits at least one solution.

By setting

$$
\widehat{L}(t, y, u)=\int_{\Omega} \widehat{G}(t, \xi, y(\xi)) \mathrm{d} \xi+\int_{\Gamma} \widehat{K}(t, \sigma, u(\sigma)) \mathrm{d} \sigma, \quad \widehat{g}(y)=\int_{\Omega} k(\xi, y(\xi)) \mathrm{d} \xi,
$$

we notice that problem $\left(\widehat{\mathcal{P}}_{t, x_{0}}\right)$ is an optimal control problem of the form of problems studied in Section 4 . However $\widehat{L}, \widehat{g}$, and $\widehat{F}$ do not satisfy assumptions of Section 2. Thus we cannot apply Theorem 4.5 to the value function $\widehat{v}$, and we cannot claim that $\widehat{v}$ is the viscosity solution to Hamilton-Jacobi-Bellman equation corresponding to $\widehat{L}, \widehat{g}$, and $\widehat{F}$. To overcome this drawback, we introduce in the next section a family of problems whose value function is locally equal to $\widehat{v}$ and is the unique viscosity solution to a Hamilton-Jacobi-Bellman equation. 


\subsection{Definition of a problem $\left(\mathcal{P}_{t, x_{0}}\right)$ equivalent to $\left(\widehat{\mathcal{P}}_{t, x_{0}}\right)$}

For all $M_{0}>0$, we set $R\left(M_{0}, T\right)=M_{0}+C(p, \tilde{p}, q, \tilde{q}, \Omega, T)\left(T^{1 / \tilde{q}} M_{U}+T^{1 / \tilde{p}} M_{f}\right)$, where $C(p, \tilde{p}, q, \tilde{q}, \Omega, T)$ is the constant appearing in (5.5). We set

$$
T_{R\left(M_{0}, T\right)}(y)=\min \left(R\left(M_{0}, T\right), \max \left(-R\left(M_{0}, T\right), y\right)\right) \quad \text { for all } y \in \mathbb{R} .
$$

In this example $Y=L^{\infty}(\Omega), Y_{0}=L^{\infty}(\Gamma), P_{M_{0}}$ (respectively $P_{M_{0}}^{0}$ ) is the projection operator from $L^{2}(\Omega)$ (respectively $L^{2}(\Gamma)$ ) into the ball in $L^{\infty}(\Omega)$ (respectively $L^{\infty}(\Gamma)$ ), centered at the origin and with radius $R\left(M_{0}, T\right)$, defined by

$$
P_{M_{0}} y(\xi)=T_{R\left(M_{0}, T\right)}(y(\xi)) \quad \text { for a.e. } \xi \in \Omega \quad\left(\text { resp. } \quad P_{M_{0}}^{0} y(\xi)=T_{R\left(M_{0}, T\right)}(y(\xi)) \quad \text { for a.e. } \xi \in \Gamma\right) .
$$

We set

$$
G(t, \xi, y(\xi))=\widehat{G}\left(t, \xi, P_{M_{0}} y(\xi)\right) \quad \text { and } \quad k(\xi, y(\xi))=\widehat{k}\left(\xi, P_{M_{0}} y(\xi)\right) \quad \text { for all } y \in L^{2}(\Omega),
$$

and $\left.h(y(\xi))=\widehat{h}\left(P_{M_{0}}^{0} y(\xi)\right)\right)$ for all $y \in L^{2}(\Gamma)$. The mappings $h, G$, and $k$ clearly depend on $M_{0}$. We have not noticed this dependence in order not to load the notation. We set

$$
L(t, y, u)=\int_{\Omega} G(t, \xi, y(\xi)) \mathrm{d} \xi+\int_{\Gamma} \widehat{K}(t, \sigma, u(\sigma)) \mathrm{d} \sigma, \quad g(y)=\int_{\Omega} k(\xi, y(\xi)) \mathrm{d} \xi, \quad \text { and } \quad F(y)=h(y) .
$$

Proposition 5.3. The mappings $L, g$ and $F$ satisfy the assumptions of Section 2 .

Proof. With $\left(\mathcal{A}_{3}\right),\left(\mathcal{A}_{4}\right)$, and the definition of $G$, for all $(t, y, u) \in[0, T] \times L^{2}(\Omega) \times L^{2}(\Gamma)$, we have:

$$
\begin{aligned}
|L(t, y, u)| & \leq \int_{\Omega}|G(t, \xi, y(\xi))| \mathrm{d} \xi+\int_{\Gamma}|\widehat{K}(t, \sigma, u(\sigma))| \mathrm{d} \sigma \\
& \leq \eta\left(R\left(M_{0}, T\right)\right)\left|\widehat{G}_{1}\right|_{L^{1}(\Omega)}+\left|\widehat{K}_{1}\right|_{L^{1}(\Gamma)}+c_{0} M_{U}^{q}=M_{L} .
\end{aligned}
$$

With $\left(\mathcal{A}_{3}\right)$ and Cauchy-Schwarz inequality, we have

$$
\begin{array}{r}
|L(t, y, u)-L(s, z, u)|=\left|\int_{\Omega}(G(s, \xi, y(\xi))-G(t, \xi, z(\xi))) \mathrm{d} \xi\right|+\left|\int_{\Gamma}(\widehat{K}(t, \sigma, u(\sigma)) \mathrm{d} \sigma-\widehat{K}(s, \sigma, u(\sigma))) \mathrm{d} \sigma\right| \\
\leq 2 \eta\left(R\left(M_{0}, T\right)\right)\left(\left|\widehat{G}_{2}\right|_{L^{2}(\Omega)}+\left|\widehat{G}_{2}\right|_{L^{1}(\Omega)}\right)\left(|y-z|_{L^{2}(\Omega)}+|t-s|^{\eta_{2}}\right)+\left(\left|\widehat{K}_{1}\right|_{L^{1}(\Gamma)}+c_{0} M_{U}^{q}\right)|t-s|^{\eta_{2}}
\end{array}
$$

that is

$$
|L(t, y, u)-L(s, z, u)| \leq K_{L}\left(|y-z|_{L^{2}(\Omega)}+|t-s|^{\eta_{2}}\right) .
$$

The estimates for $g$ can be obtained in a similar way with $\left(\mathcal{A}_{5}\right)$. The estimates for $F$ directly follows from the definition of $h$.

For all $t \in\left[0, T\left[\right.\right.$, and all $x_{0} \in L^{2}(\Omega)$, we consider the optimal control problem

$\left(\mathcal{P}_{t, x_{0}}\right) \quad \min \{J(t, u, y) \mid u \in \mathcal{M}(t, T ; U)$ and $(y, u)$ is solution of equation $(5.8)\}$,

where the cost function $J$ is defined by

$$
J(t, u, y)=\int_{t}^{T} L(s, y(s), u(s)) \mathrm{d} s+g(y(T)),
$$

and the state equation is

$$
\frac{\partial y}{\partial t}-\Delta y+y=f \quad \text { in } Q_{t, T}, \quad \frac{\partial y}{\partial n}+h(\Lambda y)=u \quad \text { on } \Sigma_{t, T}, \quad y(t)=x_{0} \quad \text { in } \Omega .
$$


Equation (5.8) can be written in the form

$$
y^{\prime}=\widehat{A} y+(-\widehat{A})^{\beta}[B u-F(\Lambda y)] \quad \text { in }(t, T), \quad y(t)=x_{0},
$$

where $\widehat{F}(t, y)=(-A)^{-\beta} f(t)+B h(y)$ satisfies assumption (iv) of Section 2. The value function of problem $\left(\mathcal{P}_{t, x_{0}}\right)$ is defined by

$$
v\left(t, x_{0}\right)=\inf _{u \in \mathcal{M}(t, T ; U)} J\left(t, y_{t, x_{0}, u}, u\right),
$$

where $y_{t, x_{0}, u}$ is solution of equation (5.8). From Theorems 3.5 and 4.5, we deduce that $v$ is the unique viscosity solution of equation (1.1) corresponding to $F, L$, and $g$.

Theorem 5.4. Let $t$ be in $\left[0, T\left[\right.\right.$ and assume that $\left|x_{0}\right|_{L^{\infty}(\Omega)} \leq M_{0}$. A pair $(y, u)$ is a solution of problem $\left(\widehat{\mathcal{P}}_{t, x_{0}}\right)$ if and only if it is a solution of $\left(\mathcal{P}_{t, x_{0}}\right)$.

Proof. If $\left|x_{0}\right|_{L^{\infty}(\Omega)} \leq M_{0}$, with Theorem 5.1 we can easily verify that, for all $u \in \mathcal{M}(t, T ; U)$, the solution $\widehat{y}_{t, x_{0}, u}$ of equation (5.2) obeys $\left\|\widehat{y}_{t, x_{0}, u}\right\|_{L^{\infty}\left(\Omega_{t, T}\right)} \leq R\left(M_{0}, T\right)$. Thus $\widehat{F}\left(\widehat{y}_{t, x_{0}, u}\right)=F\left(\widehat{y}_{t, x_{0}, u}\right)$, and $\widehat{y}_{t, x_{0}, u}$ is also the solution of (5.8). That is $\widehat{y}_{t, x_{0}, u}=y_{t, x_{0}, u}$. Thus we do not distinguish $\widehat{y}_{t, x_{0}, u}$ and $y_{t, x_{0}, u}$ up to the end of the proof. Assume that $\left(y_{t, x_{0}, \bar{u}}, \bar{u}\right)$ is a solution of $\left(\widehat{\mathcal{P}}_{t, x_{0}}\right)$, then for all $u \in \mathcal{M}(t, T ; U)$, we have

$$
J\left(t, y_{t, x_{0}, \bar{u}}, \bar{u}\right)=\widehat{J}\left(t, y_{t, x_{0}, \bar{u}}, \bar{u}\right) \leq \widehat{J}\left(t, y_{t, x_{0}, u}, u\right)=J\left(t, y_{t, x_{0}, u}, u\right),
$$

that is $\left(y_{t, x_{0}, \bar{u}}, \bar{u}\right)$ is a solution of $\left(\mathcal{P}_{t, x_{0}}\right)$. We prove that any solution of $\left(\mathcal{P}_{t, x_{0}}\right)$ is a solution of $\left(\widehat{\mathcal{P}}_{t, x_{0}}\right)$ in a similar way.

Corollary 5.5. For all $t \in\left[0, T\left[\right.\right.$ and all $x_{0}$ satisfying $\left|x_{0}\right|_{L^{\infty}(\Omega)} \leq M_{0}$, we have $\widehat{v}\left(t, x_{0}\right)=v\left(t, x_{0}\right)$.

\subsection{State equation of example 2}

Consider the following Burgers type equation in 2-D:

$$
\frac{\partial y}{\partial t}-\Delta y+\partial_{x_{1}}\left(y^{2}\right)=u \chi_{\omega} \quad \text { in } \Omega_{t, T}, \quad y=0 \quad \text { on } \Sigma_{t, T}, \quad y(t)=x_{0} \quad \text { in } \Omega .
$$

In this example, $\omega$ is an open subset in $\Omega, \chi_{\omega}$ is the characteristic function of $\omega$, and $\left(\mathcal{A}_{1}\right)$ is replaced by

$\left(\mathcal{A}_{1}^{\prime}\right) U$ is a closed bounded convex and nonempty subset in $L^{10}(\omega)$ and it obeys the condition

$$
|u|_{L^{10}(\omega)} \leq M_{U} \text { for all } u \in U .
$$

Set $X=L^{2}(\Omega), X_{\Gamma}=L^{2}(\omega), X_{0}=L^{2}(\Omega), \alpha=0$, and let $\Lambda$ be the identity in $X$. We now define the unbounded operator $A$ in $X$ by

$$
D(A)=H^{2}(\Omega) \cap H_{0}^{1}(\Omega) \quad \text { and } \quad A x=\Delta x \text { for all } x \in D(A) .
$$

Equation (5.10) can be rewritten in the form

$$
y^{\prime}=\widehat{A} y+(-A)^{\frac{1}{2}}[B u-\widehat{F}(\Lambda y)] \quad \text { in }(t, T), \quad y(t)=x_{0},
$$

where

In this example we take $\beta=\frac{1}{2}$.

$$
\widehat{F}(y)=2(-A)^{-\frac{1}{2}}\left(y \partial_{x_{1}} y\right) \quad \text { and } \quad B u=(-A)^{-\frac{1}{2}}\left(u \chi_{\omega}\right) .
$$


Theorem 5.6. For all $x_{0} \in L^{\infty}(\Omega)$ and all $u \in \mathcal{M}(t, T ; U)$, equation (5.10) admits a unique weak solution in $C\left([t, T] ; L^{2}(\Omega)\right)$. This solution belongs to $L^{\infty}(] t, T[\times \Omega)$ and it satisfies the estimate

$$
\|y\|_{L^{\infty}\left(\Omega_{t, T}\right)} \leq C(\Omega, T)\left(\left|x_{0}\right|_{L^{\infty}(\Omega)}+\left|x_{0}\right|_{L^{\infty}(\Omega)}^{3}+\|u\|_{L^{10}((t, T) \times \omega)}+\|u\|_{L^{10}((t, T) \times \omega)}^{3}\right) .
$$

Proof. It is well known that equation (5.10) admits a unique weak solution in $C\left([t, T] ; L^{2}(\Omega)\right) \cap L^{2}\left(t, T ; H_{0}^{1}(\Omega)\right)$ and that

$$
\|y\|_{C\left([t, T] ; L^{2}(\Omega)\right)}+\|y\|_{L^{2}\left(t, T ; H_{0}^{1}(\Omega)\right)} \leq 2\left|x_{0}\right|_{L^{2}(\Omega)}+C(\Omega)\|u\|_{L^{2}((t, T) \times \omega)} .
$$

If we multiply equation (5.10) by $|y|^{2 p-2} y$, and if we integrate over $(t, \tau) \times \Omega$, after integration by parts, we formally obtain:

$$
\frac{1}{2 p} \int_{\Omega}|y(\tau)|^{2 p}+\int_{t}^{\tau} \int_{\Omega}(2 p-1)|\nabla y|^{2}|y|^{2 p-2}=\frac{1}{2 p} \int_{\Omega}\left|x_{0}\right|^{2 p}+\int_{t}^{\tau} \int_{\omega} u|y|^{2 p-2} y .
$$

This identity leads to the estimate:

$$
\|y\|_{L^{\infty}\left(t, T ; L^{2 p}(\Omega)\right)} \leq C(\Omega, p)\left(\left|x_{0}\right|_{L^{2 p}(\Omega)}+\|u\|_{L^{2 p}((t, T) \times \omega)}\right) \quad \text { for all } 1 \leq p \leq 5 .
$$

This formal estimate can be justified (see [22], Th. 5). Thus we have

$$
\left\|y^{2}\right\|_{L^{\infty}\left(t, T ; L^{p}(\Omega)\right)} \leq C\left(\left|x_{0}\right|_{L^{2 p}(\Omega)}^{2}+\|u\|_{L^{2 p}((t, T) \times \omega)}^{2}\right) .
$$

Passing the term $\partial_{x_{1}}\left(y^{2}\right)$ in the right hand side of the equation and using regularity results for the heat equation, we obtain:

$$
\begin{aligned}
\|y\|_{L^{\tilde{p}}\left(t, T ; W^{1, p}(\Omega)\right)} & \leq C_{1}(\Omega, p, \tilde{p})\left(\left|x_{0}\right|_{L^{p}(\Omega)}+\|u\|_{L^{p}((t, T) \times \omega)}+\left\|y^{2}\right\|_{L^{\tilde{p}}\left(t, T ; L^{p}(\Omega)\right)}\right) \\
& \leq C_{2}(\Omega, p, \tilde{p})\left(\left|x_{0}\right|_{L^{p}(\Omega)}+\|u\|_{L^{p}((t, T) \times \omega)}+\left|x_{0}\right|_{L^{2 p}(\Omega)}^{2}+\|u\|_{L^{2 p}((t, T) \times \omega)}^{2}\right),
\end{aligned}
$$

for all $1<\tilde{p}<2$ and all $1 \leq p \leq 5$. In addition we have:

$$
\left\|y \partial_{x_{1}} y\right\|_{L^{\tilde{p}}\left(t, T ; L^{p / 2}(\Omega)\right)} \leq\|y\|_{L^{\infty}\left(t, T ; L^{p}(\Omega)\right)}\left\|\partial_{x_{1}} y\right\|_{L^{\tilde{p}}\left(t, T ; L^{p}(\Omega)\right)} .
$$

Using this estimate and regularity results for the heat equation we can write:

$$
\|y\|_{L^{\infty}\left(\Omega_{t, T}\right)} \leq\left|x_{0}\right|_{L^{\infty}(\Omega)}+C(\Omega, p, \tilde{p})\left(\|u\|_{L^{p}((t, T) \times \omega)}+\left\|y \partial_{x_{1}} y\right\|_{L^{\tilde{p}}\left(t, T ; L^{p / 2}(\Omega)\right)}\right)
$$

provided that $\frac{2}{p}+\frac{1}{\tilde{p}}<1$. Choosing $p=5, \tilde{p}=\frac{15}{8}$, and combining the previous estimates we obtain the desired result.

From Theorem 5.6, and assumption $\left(\mathcal{A}_{1}^{\prime}\right)$, we deduce

$$
\|y\|_{L^{\infty}\left(\Omega_{t, T}\right)} \leq C(\Omega, T)\left(\left|x_{0}\right|_{L^{\infty}(\Omega)}+\left|x_{0}\right|_{L^{\infty}(\Omega)}^{3}+(T-t)^{1 / 10} M_{U}+(T-t)^{3 / 10} M_{U}^{3}\right) .
$$

\subsection{Setting of the control problem for example 2}

For all $t \in\left[0, T\left[\right.\right.$, and all $x_{0} \in L^{\infty}(\Omega)$, we study the following control problem

$\left(\widehat{\mathcal{P}}_{t, x_{0}}\right)$ $\min \{\widehat{J}(t, u, y) \mid u \in \mathcal{M}(t, T ; U)$ and $(y, u)$ is solution of equation (5.10) $\}$ 
where the cost function $\widehat{J}$ is

$$
\widehat{J}(t, u, y)=\int_{t}^{T} \int_{\Omega} \widehat{G}(s, \xi, y(s, \xi)) \mathrm{d} \xi \mathrm{d} s+\int_{t}^{T} \int_{\omega} \widehat{K}(s, \xi, u(s, \xi)) \mathrm{d} \xi \mathrm{d} s+\int_{\Omega} \widehat{k}(\xi, y(T, \xi)) \mathrm{d} \xi .
$$

We assume that $\widehat{G}$ obeys $\left(\mathcal{A}_{3}\right), \widehat{k}$ obeys $\left(\mathcal{A}_{5}\right)$, and that $\widehat{K}$ obeys

$\left(\mathcal{A}_{4}^{\prime}\right)$ For all $(t, u) \in \mathbb{R}^{2}, \widehat{K}(t, \cdot, u)$ is measurable in $\omega$. For a.e. $(t, \xi) \in \mathbb{R} \times \omega, \widehat{K}(t, \xi, \cdot)$ is convex. For a.e. $\xi \in \omega, \widehat{K}(\cdot, \xi, \cdot)$ is continuous in $\mathbb{R}^{2}$ and we have the estimates:

$$
C_{\widehat{K}} \leq \widehat{K}(t, \xi, u) \leq \widehat{K}_{1}(\xi)+c_{0}|u|^{q} \quad \text { and } \quad|\widehat{K}(t, \xi, u)-\widehat{K}(s, \xi, u)| \leq\left(\widehat{K}_{1}(\xi)+c_{0}|u|^{q}\right)|t-s|^{\eta_{2}},
$$

where $q=10, \widehat{K}_{1} \in L^{1}(\omega)$.

For all $M_{0}>0$, we set

$$
R\left(M_{0}, T\right)=C(\Omega, T)\left(M_{0}+M_{0}^{3}+T^{1 / 10} M_{U}+T^{3 / 10} M_{U}^{3}\right),
$$

and we define the truncated problem in a similar way as in example 1, with obvious modifications. More precisely, $Y, P_{M_{0}}, G, k$, and $g$ are defined as in Example 1,

$$
L(t, y, u)=\int_{\Omega} G(t, \xi, y(\xi)) \mathrm{d} \xi+\int_{\omega} \widehat{K}(t, \xi, u(\xi)) \mathrm{d} \xi, \quad \text { and } \quad F(y)=2(-A)^{-\frac{1}{2}}\left(P_{M_{0}} y \partial_{x_{1}} P_{M_{0}} y\right),
$$

for all $y \in L^{2}(\Omega)$. We can take any $\beta_{0}$ in $(0,1 / 2)$. For example setting $\beta_{0}=1 / 4$, we have

$$
|F(y)|_{D\left((-A)^{\frac{1}{4}}\right)} \leq C\left|\left(P_{M_{0}} y\right)^{2}\right|_{D\left((-A)^{\frac{1}{4}}\right)} \leq C|y|_{D\left((-A)^{\frac{1}{2}}\right)}^{2} \quad \text { for all } y \in D\left((-A)^{\frac{1}{2}}\right)=H_{0}^{1}(\Omega),
$$

and the additional condition in assumption (iv) when $\beta=\frac{1}{2}$ is satisfied. The other conditions in assumption (iv) are also satisfied. We can define $\left(\mathcal{P}_{t, x_{0}}\right)$ similarly as in Example 1. In particular the state equation for $\left(\mathcal{P}_{t, x_{0}}\right)$ is

$$
y^{\prime}=A y+(-A)^{\frac{1}{2}}[B u-F(\Lambda y)] \quad \text { in }(t, T), \quad y(t)=x_{0} .
$$

Denoting by $\widehat{v}\left(t, x_{0}\right)$ the value function of problem $\left(\widehat{\mathcal{P}}_{t, x_{0}}\right)$, and by $v\left(t, x_{0}\right)$ the value function of problem $\left(\mathcal{P}_{t, x_{0}}\right)$, as in example 1 , we can prove the following Theorem.

Theorem 5.7. The value function $v\left(t, x_{0}\right)$ is the unique viscosity solution of the Hamilton-Jacobi-Bellman equation (1.1) associated with $\left(\mathcal{P}_{t, x_{0}}\right)$. For all $t \in\left[0, T\left[\right.\right.$ and all $x_{0} \in X$ satisfying $\left|x_{0}\right|_{L^{\infty}(\Omega)} \leq M_{0}$, we have $\widehat{v}\left(t, x_{0}\right)=v\left(t, x_{0}\right)$.

\subsection{Example 3}

We consider the same equation as in Example 2, and now $\left(\mathcal{A}_{1}\right)$ is replaced by

$\left(\mathcal{A}_{1}^{\prime \prime}\right) U$ is a closed bounded convex and nonempty subset in $L^{8}(\omega)$ and it obeys the condition

$$
|u|_{L^{8}(\omega)} \leq M_{U} \text { for all } u \in U .
$$

Set $X=L^{2}(\Omega), X_{\Gamma}=L^{2}(\omega), X_{0}=H_{0}^{3 / 4}(\Omega), \alpha=\frac{3}{8}$, and let $\Lambda$ be the identity in $X_{0}$ (thus $\Lambda$ is considered as an unbounded operator in $X)$. We define the unbounded operator $(A, D(A))$ in $X$ as in Example 2. Equation (5.10) can be rewritten in the form

$$
y^{\prime}=A y+(-A)^{\frac{\varepsilon^{\prime}}{2}}[B u-\widehat{F}(y)] \quad \text { in }(t, T), \quad y(t)=x_{0},
$$


where

$$
\widehat{F}(y)=2(-A)^{-\frac{\varepsilon^{\prime}}{2}}\left(y \partial_{x_{1}} y\right) \quad \text { and } \quad B u=(-A)^{-\frac{\varepsilon^{\prime}}{2}}\left(u \chi_{\omega}\right), \quad \text { with } 0 \leq \varepsilon^{\prime} \leq 1 .
$$

In this example, we take $\beta=\frac{\varepsilon^{\prime}}{2}$ (below we choose $\varepsilon^{\prime}=5 / 8$ ).

Theorem 5.8. For all $x_{0} \in H_{0}^{3 / 4}(\Omega)$ and all $u \in \mathcal{M}(t, T ; U)$, equation (5.10) admits a unique weak solution in $C\left([t, T] ; H_{0}^{3 / 4}(\Omega)\right)$, and it satisfies the estimate

$$
\|y\|_{C\left([t, T] ; H_{0}^{3 / 4}(\Omega)\right)} \leq C(\Omega, T)\left(\left|x_{0}\right|_{H_{0}^{3 / 4}(\Omega)}+\left|x_{0}\right|_{H_{0}^{3 / 4}(\Omega)}^{3}+\|u\|_{L^{8}((t, T) \times \omega)}+\|u\|_{L^{8}((t, T) \times \omega)}^{3}\right) .
$$

Proof. Observe that $H_{0}^{3 / 4}(\Omega) \hookrightarrow L^{8}(\Omega)$. As in Example 2, we have

$$
\begin{aligned}
& \left\|y^{2}\right\|_{L^{\infty}\left(t, T ; L^{p}(\Omega)\right)} \leq C\left(\left|x_{0}\right|_{L^{2 p}(\Omega)}^{2}+\|u\|_{L^{2 p}((t, T) \times \omega)}^{2}\right), \\
& \|y\|_{L^{\tilde{p}}\left(t, T ; W^{1, p}(\Omega)\right)} \leq C_{2}(\Omega, p, \tilde{p})\left(\left|x_{0}\right|_{L^{p}(\Omega)}+\|u\|_{L^{2 p}((t, T) \times \omega)}+\left|x_{0}\right|_{L^{2 p}(\Omega)}^{2}+\|u\|_{L^{2 p}((t, T) \times \omega)}^{2}\right),
\end{aligned}
$$

for all $1<\tilde{p}<2$ and all $1<p \leq 4$, and

$$
\left\|y \partial_{x_{1}} y\right\|_{L^{\tilde{p}}\left(t, T ; L^{p / 2}(\Omega)\right)} \leq\|y\|_{L^{\infty}\left(t, T ; L^{p}(\Omega)\right)}\left\|\partial_{x_{1}} y\right\|_{L^{\tilde{p}}\left(t, T ; L^{p}(\Omega)\right)} \quad \text { if in addition } 2 \leq p \leq 4 .
$$

Using this estimate and regularity results for the heat equation we can write:

$$
\|y\|_{C\left([t, T] ; H_{0}^{3 / 4}(\Omega)\right)} \leq C(\Omega, \tilde{p})\left(\left|x_{0}\right|_{H_{0}^{3 / 4}(\Omega)}+\|u\|_{L^{2}((t, T) \times \omega)}+\left\|y \partial_{x_{1}} y\right\|_{L^{\tilde{p}}\left(t, T ; L^{2}(\Omega)\right)}\right),
$$

if $\frac{1}{\tilde{p}}<\frac{5}{8}$. Thus, choosing $p=4, \tilde{p}=\frac{16}{9}$, and combining the above estimates, we obtain:

$$
\|y\|_{C\left([t, T] ; H_{0}^{3 / 4}(\Omega)\right)} \leq C(\Omega, T)\left(\left|x_{0}\right|_{H_{0}^{3 / 4}(\Omega)}+\left|x_{0}\right|_{H_{0}^{3 / 4}(\Omega)}^{3}+\|u\|_{L^{8}((t, T) \times \omega)}+\|u\|_{L^{8}((t, T) \times \omega)}^{3}\right) .
$$

From Theorem 5.8, and assumptions $\left(\mathcal{A}_{1}^{\prime \prime}\right)$ and $\left(\mathcal{A}_{5}\right)$, we deduce

$$
\|y\|_{C\left([t, T] ; H_{0}^{3 / 4}(\Omega)\right)} \leq C(\Omega, T)\left(\left|x_{0}\right|_{H_{0}^{3 / 4}(\Omega)}+\left|x_{0}\right|_{H_{0}^{3 / 4}(\Omega)}^{3}+(T-t)^{1 / 8} M_{U}+(T-t)^{3 / 8} M_{U}^{3}\right) .
$$

Now we set

$$
R\left(M_{0}, T\right)=C(\Omega, T)\left(M_{0}+M_{0}^{3}+T^{1 / 8} M_{U}+T^{3 / 8} M_{U}^{3}\right) .
$$

Let us denote by $P_{M_{0}}^{0}$ the orthogonal projection in $H_{0}^{3 / 4}(\Omega)$ on the ball centered at zero and with radius $R\left(M_{0}, T\right)$. Let us set

$$
F(y)=2(-A)^{-\frac{\varepsilon^{\prime}}{2}}\left(P_{M_{0}}^{0} y \partial_{x_{1}}\left(P_{M_{0}}^{0} y\right)\right) \quad \text { for all } y \in H_{0}^{3 / 4}(\Omega)
$$

Let us show that $F$ is bounded and Lipschitz from $H_{0}^{3 / 4}(\Omega)$ into $X$. We have

$$
|F(y)|_{L^{2}(\Omega)}=2\left|P_{M_{0}}^{0} y \partial_{x_{1}}\left(P_{M_{0}}^{0} y\right)\right|_{H^{-\varepsilon^{\prime}}(\Omega)}=2\left|P_{M_{0}}^{0} y \partial_{x_{1}}\left(P_{M_{0}}^{0} y\right)\right|_{B_{2,2}^{-\varepsilon^{\prime}}(\Omega)} .
$$

Using the product estimate [25], page 171

$$
B_{p_{1}, q_{1}}^{s_{1}}(\Omega) \cdot B_{p_{2}, q_{2}}^{s_{2}}(\Omega) \hookrightarrow B_{p, q}^{s_{1}}(\Omega),
$$

with

$$
s_{1}=-\varepsilon^{\prime}=-\frac{5}{8}, \quad s_{2}=\varepsilon=\frac{3}{4}, \quad p_{1}=\frac{32}{11}, \quad p_{2}=p=2, \quad q_{1}=q_{2}=q=2
$$


we have

Recall that

$$
|F(y)|_{L^{2}(\Omega)} \leq C\left|P_{M_{0}}^{0} y\right|_{B_{2,2}^{\varepsilon}(\Omega)}\left|\partial_{x_{1}}\left(P_{M_{0}}^{0} y\right)\right|_{B_{p_{1}, 2}^{-\varepsilon^{\prime}}(\Omega)} .
$$

$$
B_{2,2}^{\varepsilon}(\Omega)=H^{\varepsilon}(\Omega), \quad B_{2,2}^{-\varepsilon^{\prime}}(\Omega)=H^{-\varepsilon^{\prime}}(\Omega), \quad \text { and } \quad B_{p_{1}, 2}^{-\varepsilon^{\prime}}(\Omega)=\left(B_{p_{1}^{\prime}, 2}^{\varepsilon^{\prime}}(\Omega)\right)^{\prime}
$$

where $p_{1}^{\prime}=p_{1} /\left(p_{1}-1\right)$. Moreover

$$
B_{p_{1}^{\prime}, 2}^{\varepsilon^{\prime}}(\Omega) \hookrightarrow B_{p_{1}^{\prime}, p_{1}^{\prime}}^{\varepsilon^{\prime \prime}}(\Omega)=W^{\varepsilon^{\prime \prime}, p_{1}^{\prime}}(\Omega) \hookrightarrow H^{\hat{\varepsilon}}(\Omega)
$$

for all $\hat{\varepsilon}<\varepsilon^{\prime \prime}<\varepsilon^{\prime}$, and if $2=\frac{2 p_{1}^{\prime}}{2-\left(\varepsilon^{\prime \prime}-\hat{\varepsilon}\right) p_{1}^{\prime}}$. We choose $\hat{\varepsilon}=\frac{1}{4}$ and $\varepsilon^{\prime \prime}=\frac{9}{16}$, we have $H^{-\hat{\varepsilon}}(\Omega) \hookrightarrow B_{p_{1}, 2}^{-\varepsilon^{\prime}}(\Omega)$, and we obtain

$$
|F(y)|_{L^{2}(\Omega)} \leq C\left|P_{M_{0}}^{0} y\right|_{H_{0}^{3 / 4}(\Omega)}\left|\partial_{x_{1}}\left(P_{M_{0}}^{0} y\right)\right|_{H^{-\hat{\varepsilon}}(\Omega)} \leq C\left|P_{M_{0}}^{0} y\right|_{H_{0}^{3 / 4}(\Omega)}\left|P_{M_{0}}^{0} y\right|_{H_{0}^{3 / 4}(\Omega)} .
$$

Thus $F$ is bounded from $X_{0}$ into $X$. Let us verify that $F$ is Lipschitz. We have

$$
\begin{aligned}
|F(y)-F(z)|_{L^{2}(\Omega)} & \leq 2\left|P_{M_{0}}^{0} y \partial_{x_{1}}\left(P_{M_{0}}^{0} y\right)-P_{M_{0}}^{0} z \partial_{x_{1}}\left(P_{M_{0}}^{0} z\right)\right|_{H^{-\varepsilon^{\prime}}(\Omega)} \\
& \leq 2\left|P_{M_{0}}^{0} y \partial_{x_{1}}\left(P_{M_{0}}^{0} y-P_{M_{0}}^{0} z\right)\right|_{H^{-\varepsilon^{\prime}}(\Omega)}+2\left|P_{M_{0}}^{0} z \partial_{x_{1}}\left(P_{M_{0}}^{0} y-P_{M_{0}}^{0} z\right)\right|_{H^{-\varepsilon^{\prime}}(\Omega)} \\
& \leq C\left(\left|P_{M_{0}}^{0} y\right|_{H_{0}^{3 / 4}(\Omega)}+\left|P_{M_{0}}^{0} y\right|_{H_{0}^{3 / 4}(\Omega)}\right)\left|P_{M_{0}}^{0} y-P_{M_{0}}^{0} z\right|_{H_{0}^{3 / 4}(\Omega)} \\
& \leq C\left(\left|P_{M_{0}}^{0} y\right|_{H_{0}^{3 / 4}(\Omega)}+\left|P_{M_{0}}^{0} y\right|_{H_{0}^{3 / 4}(\Omega)}\right)|y-z|_{H_{0}^{3 / 4}(\Omega)} .
\end{aligned}
$$

To obtain the last inequality we have used the Lipschitz continuity of $P_{M_{0}}^{0}$ from $H_{0}^{3 / 4}(\Omega)$ into itself. We define $\left(\widehat{\mathcal{P}}_{t, x_{0}}\right)$ as in Example 2, and we assume that $\widehat{G}$ obeys $\left(\mathcal{A}_{3}^{\prime \prime}\right), \widehat{k}$ obeys $\left(\mathcal{A}_{5}^{\prime \prime}\right)$, and $\widehat{K}$ obeys $\left(\mathcal{A}_{4}^{\prime \prime}\right)$, where $\left(\mathcal{A}_{3}^{\prime \prime}\right)$, $\left(\mathcal{A}_{5}^{\prime \prime}\right)$, and $\left(\mathcal{A}_{4}^{\prime \prime}\right)$ respectively correspond to $\left(\mathcal{A}_{3}\right),\left(\mathcal{A}_{5}\right)$, and $\left(\mathcal{A}_{4}^{\prime}\right)$, where $\eta(|y|)=|y|^{r}, 1 \leq r \leq 4, \widehat{G}_{1} \in L^{\rho_{1}}(\Omega)$, $\widehat{G}_{2} \in L^{\rho_{2}}(\Omega), \widehat{k}_{1} \in L^{\rho_{1}}(\Omega), \widehat{k}_{2} \in L^{\rho_{2}}(\Omega)$, and $q=8$, with $\frac{r}{8}+\frac{1}{\rho_{1}}=1$ and $\frac{r}{8}+\frac{1}{\rho_{2}}=\frac{1}{2}$. We define $\left(\widehat{\mathcal{P}}_{t, x_{0}}\right)$ as in Example 2. Next we define $\left(\mathcal{P}_{t, x_{0}}\right)$ with the state equation

$$
y^{\prime}=A y+(-A)^{\frac{\varepsilon^{\prime}}{2}}[B u-F(\Lambda y)] \quad \text { in }(t, T), \quad y(t)=x_{0},
$$

and with $G, k, g$, and $L$ defined as in example 2, but where $P_{M_{0}}$ is now the projection in $L^{2}(\Omega)$ on the ball in $H_{0}^{3 / 4}(\Omega)$ centered at zero and with radius $R\left(M_{0}, T\right)$. Recall that $P_{M_{0}}$ is Lipschitz continuous from $L^{2}(\Omega)$ into itself. Since the embedding from $H_{0}^{3 / 4}(\Omega)$ into $L^{8}(\Omega)$ is continuous, with assumptions $\left(\mathcal{A}_{3}^{\prime \prime}\right),\left(\mathcal{A}_{5}^{\prime \prime}\right)$, and $\left(\mathcal{A}_{4}^{\prime \prime}\right)$, we easily verify that assumptions of Section 2 are satisfied by $L, g$, and $F$. Denoting by $\widehat{v}\left(t, x_{0}\right)$ the value function of problem $\left(\widehat{\mathcal{P}}_{t, x_{0}}\right)$, and by $v\left(t, x_{0}\right)$ the value function of problem $\left(\mathcal{P}_{t, x_{0}}\right)$, we can prove the following Theorem.

Theorem 5.9. The value function $v\left(t, x_{0}\right)$ is the unique viscosity solution of the Hamilton-Jacobi-Bellman equation (1.1) associated with $\left(\mathcal{P}_{t, x_{0}}\right)$. For all $t \in\left[0, T\left[\right.\right.$ and all $x_{0}$ satisfying $\left|x_{0}\right|_{H_{0}^{3 / 4}(\Omega)} \leq M_{0}$, we have $\widehat{v}\left(t, x_{0}\right)=v\left(t, x_{0}\right)$.

\section{REFERENCES}

[1] H. Amann, Linear and quasilinear parabolic problems. Vol. I, Abstract linear theory. Birkhäuser Boston Inc., Boston, MA. Monographs Math. 89 (1995).

[2] V. Barbu and G. Da Prato, Hamilton-Jacobi equations in Hilbert spaces, Pitman (Advanced Publishing Program), Boston, MA Res. Notes Math. 86 (1983).

[3] A. Bensoussan, G. Da Prato, M.C. Delfour and S.K. Mitter, Representation and control of infinite-dimensional systems. Vol. 1. Systems \& Control: Foundations \& Applications. Birkhäuser Boston Inc., Boston, MA (1992).

[4] A. Bensoussan, G. Da Prato, M.C. Delfour and S.K. Mitter, Representation and control of infinite-dimensional systems. Vol. 2. Systems \& Control: Foundations \& Applications. Birkhäuser Boston Inc., Boston, MA (1993). 
[5] P. Cannarsa and H. Frankowska, Value function and optimality condition for semilinear control problems. II. Parabolic case. Appl. Math. Optim. 33 (1996) 1-33.

[6] P. Cannarsa and M.E. Tessitore, Cauchy problem for the dynamic programming equation of boundary control. Boundary control and variation (1994) 13-26.

[7] P. Cannarsa and M.E. Tessitore, Cauchy problem for Hamilton-Jacobi equations and Dirichlet boundary control problems of parabolic type, in Control of partial differential equations and applications (Laredo, 1994), Dekker, New York. Lect. Notes Pure Appl. Math. 174 (1996) 31-42.

[8] P. Cannarsa and M.E. Tessitore, Dynamic programming equation for a class of nonlinear boundary control problems of parabolic type. Cont. Cybernetics 25 (1996) 483-495. Distributed parameter systems: modelling and control (1995).

[9] P. Cannarsa and M.E. Tessitore, Infinite-dimensional Hamilton-Jacobi equations and Dirichlet boundary control problems of parabolic type. SIAM J. Control Optim. 34 (1996) 1831-1847.

[10] M.G. Crandall and P.-L. Lions, Hamilton-Jacobi equations in infinite dimensions. I. Uniqueness of viscosity solutions. J. Funct. Anal. 62 (1985) 379-396.

[11] M.G. Crandall and P.-L. Lions, Hamilton-Jacobi equations in infinite dimensions. II. Existence of viscosity solutions. J. Funct. Anal. 65 (1986) 368-405.

[12] M.G. Crandall and P.-L. Lions, Hamilton-Jacobi equations in infinite dimensions. III. J. Funct. Anal. 68 (1986) $214-247$.

[13] M.G. Crandall and P.-L. Lions, Viscosity solutions of Hamilton-Jacobi equations in infinite dimensions. IV. Hamiltonians with unbounded linear terms. J. Funct. Anal. 90 (1990) 237-283.

[14] M.G. Crandall and P.-L. Lions, Viscosity solutions of Hamilton-Jacobi equations in infinite dimensions. V. Unbounded linear terms and B-continuous solutions. J. Funct. Anal. 97 (1991) 417-465.

[15] M.G. Crandall and P.-L. Lions, Hamilton-Jacobi equations in infinite dimensions. VI. Nonlinear $A$ and Tataru's method refined, in Evolution equations, control theory, and biomathematics (Han sur Lesse 1991), Dekker, New York. Lect. Notes Pure Appl. Math. 155 (1994) 51-89.

[16] M.G. Crandall and P.-L. Lions, Viscosity solutions of Hamilton-Jacobi equations in infinite dimensions. VII. The HJB equation is not always satisfied. J. Funct. Anal. 125 (1994) 111-148.

[17] S Gombao, Équations de Hamilton-Jacobi-Bellman pour des problèmes de contrôle d'équations paraboliques semi-linéaires. Approche théorique et numérique. Université Paul Sabatier, Toulouse (2004).

[18] F. Gozzi, S.S. Sritharan and A. Święch, Viscosity solutions of dynamic-programming equations for the optimal control of the two-dimensional Navier-Stokes equations. Arch. Ration. Mech. Anal. 163 (2002) 295-327.

[19] D. Henry, Geometric theory of semilinear parabolic equations, Springer-Verlag, Berlin. Lect. Notes Math. 840 (1981).

[20] H. Ishii, Viscosity solutions for a class of Hamilton-Jacobi equations in Hilbert spaces. J. Funct. Anal. 105 (1992) $301-341$.

[21] J.-L. Lions and E. Magenes, Problèmes aux limites non homogènes et applications. Vol. 1. Travaux et Recherches Mathématiques, No. 17. Dunod, Paris (1968).

[22] S.M. Rankin, III. Semilinear evolution equations in Banach spaces with application to parabolic partial differential equations. Trans. Amer. Math. Soc. 336 (1993) 523-535.

[23] J.-P. Raymond, Nonlinear boundary control of semilinear parabolic problems with pointwise state constraints. Discrete Contin. Dynam. Syst. 3 (1997) 341-370.

[24] J.-P. Raymond and H. Zidani, Hamiltonian Pontryagin's principles for control problems governed by semilinear parabolic equations. Appl. Math. Optim. 39 (1999) 143-177.

[25] T. Runst and W. Sickel, Sobolev spaces of fractional order, Nemytskij operators, and nonlinear partial differential equations, Walter de Gruyter \& Co., Berlin, de Gruyter Series in Nonlinear Analysis and Applications 3 (1996).

[26] K. Shimano, A class of Hamilton-Jacobi equations with unbounded coefficients in Hilbert spaces. Appl. Math. Optim. 45 (2002) $75-98$.

[27] H.M. Soner, On the Hamilton-Jacobi-Bellman equations in Banach spaces. J. Optim. Theory Appl. 57 (1988) $429-437$.

[28] D. Tataru, Viscosity solutions for the dynamic programming equations. Appl. Math. Optim. 25 (1992) 109-126.

[29] D. Tataru, Viscosity solutions for Hamilton-Jacobi equations with unbounded nonlinear term: a simplified approach. J. Differ. Equ. 111 (1994) 123-146. 